\title{
Taste-Active Maillard Reaction Products in Roasted Garlic (Allium Sativum)
}

Junichiro Wakamatsu, Timo D. Stark, Thomas Hofmann

Chair of Food Chemistry and Molecular Sensory Science, Technische Universität München, Lise-Meitner Str. 34, D-85354, Freising, Germany 


\section{List of Supporting Information}

1. Analytical conditions for purification of crude fractions (GS-1, 2, 3, $4,5$ and 6$)$

Table S1. Analytical conditions for HPLC purifications and retention times for collected peaks

2. MS and NMR data of compounds 1-9 acortatarin $\mathrm{A}$ (compound 1)

Figure S1. HRESIMS (positive) spectrum

Figure S2. Elemental composition report of the precursor ion

Figure S3. ${ }^{1} \mathrm{H}$ NMR spectrum (DMSO- $d_{6}, 400 \mathrm{MHz}$ )

Figure S4. ${ }^{13} \mathrm{C}$ NMR spectrum (DMSO- $d_{6}, 100 \mathrm{MHz}$ )

Figure S5. COSY correlations

Figure S6. HSQC correlations

Figure S7. HMBC correlations

pollenopyrroside A (compound 2)

Figure S8. HRESIMS (positive) spectrum

Figure S9. Elemental composition report of the precursor ion

Figure S10. ${ }^{1} \mathrm{H}$ NMR spectrum (DMSO- $d_{6}, 500 \mathrm{MHz}$ )

Figure $S 11 .{ }^{13} \mathrm{C}$ NMR spectrum (DMSO- $d_{6}, 125 \mathrm{MHz}$ )

Figure S12. COSY correlations

Figure S13. HSQC correlations

Figure S14. HMBC correlations

epi-acortatarin A (compound 3)

Figure S15. HRESIMS (positive) spectrum

Figure S16. Elemental composition report of the precursor ion

Figure S17. ${ }^{1} \mathrm{H}$ NMR spectrum (DMSO- $d_{6}, 400 \mathrm{MHz}$ )

Figure $S 18 .{ }^{13} \mathrm{C}$ NMR spectrum (DMSO- $d_{6}, 100 \mathrm{MHz}$ ) 
Figure S19. COSY correlations

Figure S20. HSQC correlations

Figure S21. HMBC correlations

xylapyrroside A (compound 4)

Figure S22. HRESIMS (positive) spectrum

Figure S23. Elemental composition report of the precursor ion

Figure S24. ${ }^{1} \mathrm{H}$ NMR spectrum (DMSO- $d_{6}, 400 \mathrm{MHz}$ )

Figure $S 25 .{ }^{13} \mathrm{C}$ NMR spectrum (DMSO- $d_{6}, 100 \mathrm{MHz}$ )

Figure S26. COSY correlations

Figure S27. HSQC correlations

Figure S28. HMBC correlations

5-hydroxymethyl-1-[(5-hydroxymethyl-2-furanyl)methyl]-1 $H$-pyrrole-2 -carbaldehyde (compound 5)

Figure S29. HRESIMS (positive) spectrum

Figure S30. Elemental composition report of the precursor ion

Figure S31. ${ }^{1} \mathrm{H}$ NMR spectrum (DMSO- $d_{6}, 400 \mathrm{MHz}$ )

Figure $S 32 .{ }^{13} \mathrm{C}$ NMR spectrum (DMSO- $d_{6}, 100 \mathrm{MHz}$ )

Figure S33. COSY NMR correlations

Figure S34. HSQC NMR correlations

Figure S35. HMBC NMR correlations

3-(allylthio)-2-(2-formyl-5-hydroxymethyl-1 H-pyrrol-1-yl)propanoic acid (compound 6)

Figure S36. HRESIMS (negative) spectrum

Figure S37. Elemental composition report of the precursor ion

Figure S38. ${ }^{1} \mathrm{H}$ NMR spectrum (DMSO- $d_{6}, 400 \mathrm{MHz}$ )

Figure S39. ${ }^{13} \mathrm{C}$ NMR spectrum (DMSO- $d_{6}, 100 \mathrm{MHz}$ )

Figure S40. COSY correlations

Figure S41. HSQC correlations

Figure S42. HMBC correlations 
(4S)-4-allylthiomethyl-3,4-dihydro-3-oxo-1 $H$-pyrrolo[2,1-c][1,4]oxazin e-6-carbaldehyde (compound 7)

Figure S43. HRESIMS (negative) spectrum

Figure S44. Elemental composition report of the precursor ion

Figure S45. ${ }^{1} \mathrm{H}$ NMR spectrum (DMSO- $d_{6}, 500 \mathrm{MHz}$ )

Figure $S 46 .{ }^{13} \mathrm{C}$ NMR spectrum (DMSO- $d_{6}, 125 \mathrm{MHz}$ )

Figure S47. COSY correlations

Figure S48. HSQC correlations

Figure S49. HMBC correlations

(2R)-3-(allylthio)-2-((4R)-4-(allylthiomethyl)-6-formyl-3-oxo-3,4-dihyd ropyrrolo[1,2-a]pyrazin-2(1H)-yl)propanoic acid (compound 8)

Figure S50 Chromatograms of compounds 8 and 9 recorded by UV detection (290 nm) using UPLC-TOF-MS

Figure S51. HRESIMS (negative) spectrum

Figure S52. Elemental composition report of the precursor ion

Figure S53. ${ }^{1} \mathrm{H}$ NMR spectrum (DMSO- $d_{6}, 500 \mathrm{MHz}$ )

Figure S54. ${ }^{13} \mathrm{C}$ NMR spectrum (DMSO- $d_{6}, 125 \mathrm{MHz}$ )

Figure S55. COSY correlations

Figure S56. HSQC correlations

Figure S57. HMBC correlations

(2R)-3-(allylthio)-2-((4S)-4-(allylthiomethyl)-6-formyl-3-oxo-3,4-dihydr opyrrolo[1,2-a]pyrazin-2(1H)-yl)propanoic acid (compound 9)

Figure S58. HRESIMS (negative) spectrum

Figure S59. Elemental composition report of the precursor ion

Figure S60. ${ }^{1} \mathrm{H}$ NMR spectrum (DMSO- $d_{6}, 500 \mathrm{MHz}$ )

Figure $S 61 .{ }^{13} \mathrm{C}$ NMR spectrum (DMSO- $d_{6}, 125 \mathrm{MHz}$ )

Figure S62. COSY correlations

Figure S63. HSQC correlations

Figure S64. HMBC correlations 
2-(allylthioethyl)-4-(allylthiomethyl)-3-oxo-3,4-dihydropyrrolo[1,2a]pyr azin-2(1H)-6-carbaldehyde (racemate of compound 10 and 11)

Figure S64. HRESIMS (negative) spectrum

Figure S65. Elemental composition report of the precursor ion

Figure S66. ${ }^{1} \mathrm{H}$ NMR spectrum (DMSO- $d_{6}, 500 \mathrm{MHz}$ )

Figure S67. ${ }^{13} \mathrm{C}$ NMR spectrum (DMSO- $d_{6}, 125 \mathrm{MHz}$ )

Figure S68. COSY correlations

Figure S69. HSQC correlations

Figure S70. HMBC correlations

Figure S71. CD spectra of Maillard reaction product 7 (green) and synthesized references 10 (blue) and $\mathbf{1 1}$ (red)

3. Analytical conditions for quantitative analysis on identified compounds in garlic preparations

Table S2. UPLC-MS/MS (ESI) parameters for quantitative analysis on identified compounds. 
1. Analytical conditions for purification of crude fractions (GS-1, 2, 3, 4, 5 and 6)

\begin{tabular}{|c|c|c|}
\hline & Gradient conditions & Collected peaks \\
\hline GS-1 & $\begin{array}{c}12.5 \% \mathrm{~B} \rightarrow 12.5 \% \mathrm{~B} \rightarrow 18 \% \mathrm{~B} \rightarrow 95 \% \mathrm{~B} \\
0 \mathrm{~min} \quad 3 \mathrm{~min} \quad 15 \mathrm{~min} \quad 17.5 \mathrm{~min}\end{array}$ & 10.3 min (Compound.1) \\
\hline GS-2 & $\begin{array}{c}12.5 \% \mathrm{~B} \rightarrow 12.5 \% \mathrm{~B} \rightarrow 18 \% \mathrm{~B} \rightarrow 95 \% \mathrm{~B} \\
0 \mathrm{~min} \quad 3 \mathrm{~min} \quad 15 \mathrm{~min} \quad 17.5 \mathrm{~min}\end{array}$ & $\begin{array}{l}\text { 11.4 } \min \text { (Compound.2), } 12.4 \text { min (Compound.3), } \\
\text { 13.3 min (Compound.4), 14.8 min (Compound.5) }\end{array}$ \\
\hline GS-3 & $\begin{array}{c}30 \% \mathrm{~B} \rightarrow 30 \% \mathrm{~B} \rightarrow 80 \% \mathrm{~B} \\
0 \mathrm{~min} \quad 3 \mathrm{~min} \quad 18 \mathrm{~min}\end{array}$ & 7.6 min (Compound.6) \\
\hline GS-4 & $\begin{array}{r}30 \% \mathrm{~B} \rightarrow 30 \% \mathrm{~B} \rightarrow 80 \% \mathrm{~B} \\
0 \mathrm{~min} \quad 3 \mathrm{~min} \quad 18 \mathrm{~min}\end{array}$ & $12.8 \min$ (Compound.7) \\
\hline GS-5 & $\begin{array}{r}40 \% \mathrm{~B} \rightarrow 40 \% \mathrm{~B} \rightarrow 80 \% \mathrm{~B} \\
0 \mathrm{~min} \quad 3 \mathrm{~min} \quad 20 \mathrm{~min}\end{array}$ & 11.4 min (Compound.8) \\
\hline GS-6 & $\begin{array}{c}30 \% \mathrm{~B} \rightarrow 30 \% \mathrm{~B} \rightarrow 80 \% \mathrm{~B} \\
0 \mathrm{~min} \quad 3 \mathrm{~min} \quad 20 \mathrm{~min}\end{array}$ & 14.9 min (Compound.9) \\
\hline
\end{tabular}

Table S1. Analytical conditions for HPLC purifications and retention times for collected peaks. 


\section{MS and NMR data of compound 1-9}

\section{Acortatarin A (1)}

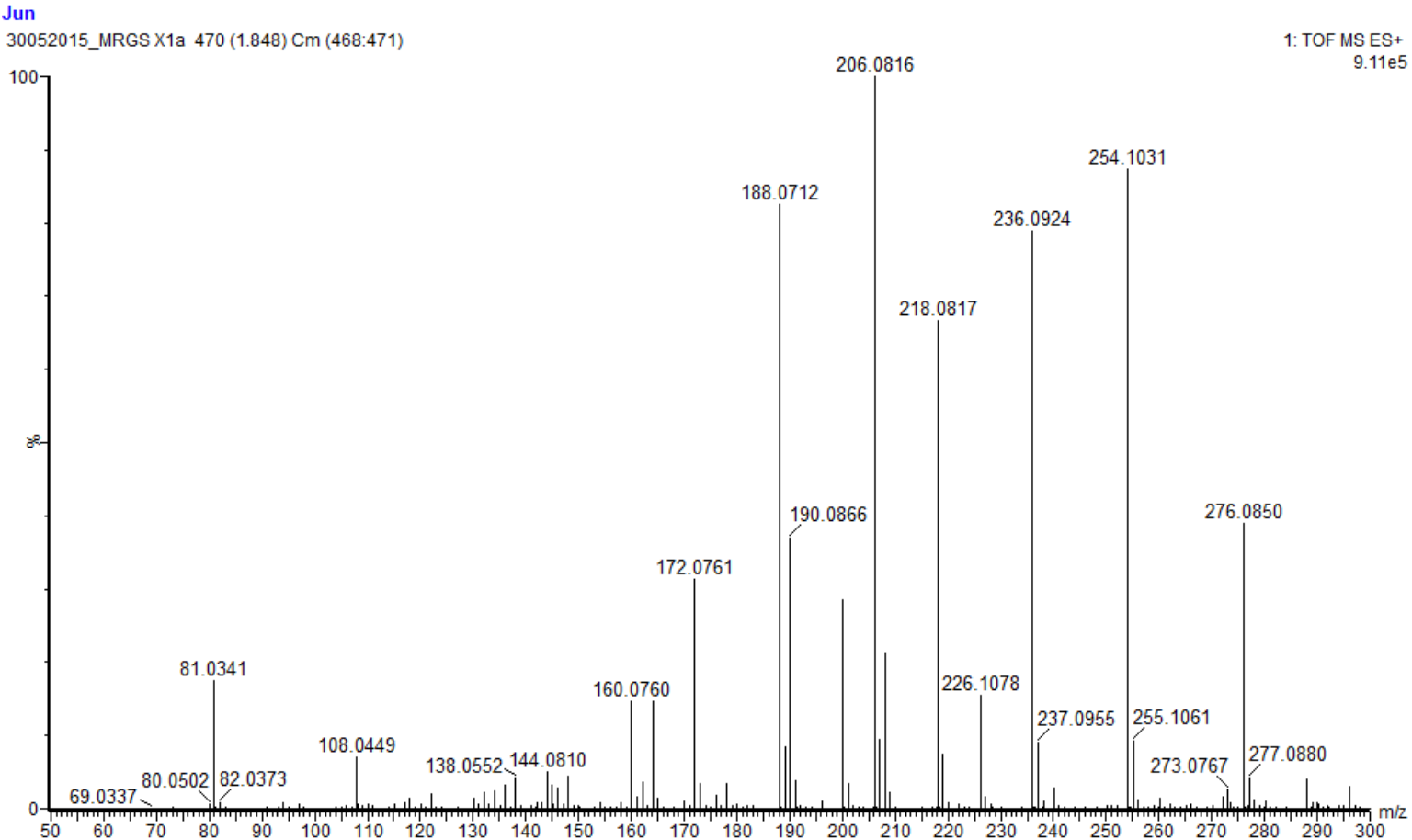

Figure S1. HRESIMS (positive) spectra

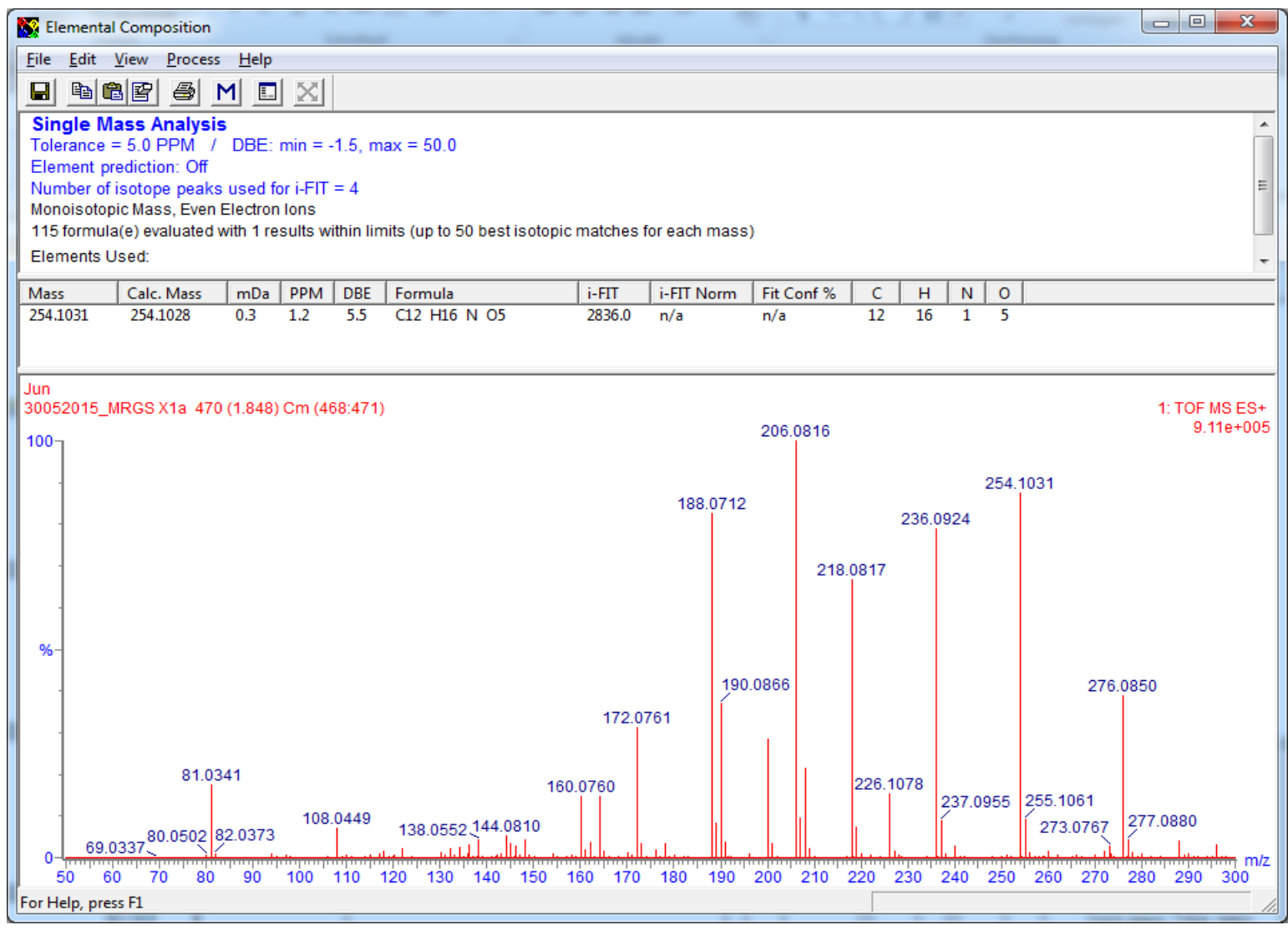

Figure S2. Elemental composition report of the precursor ion 


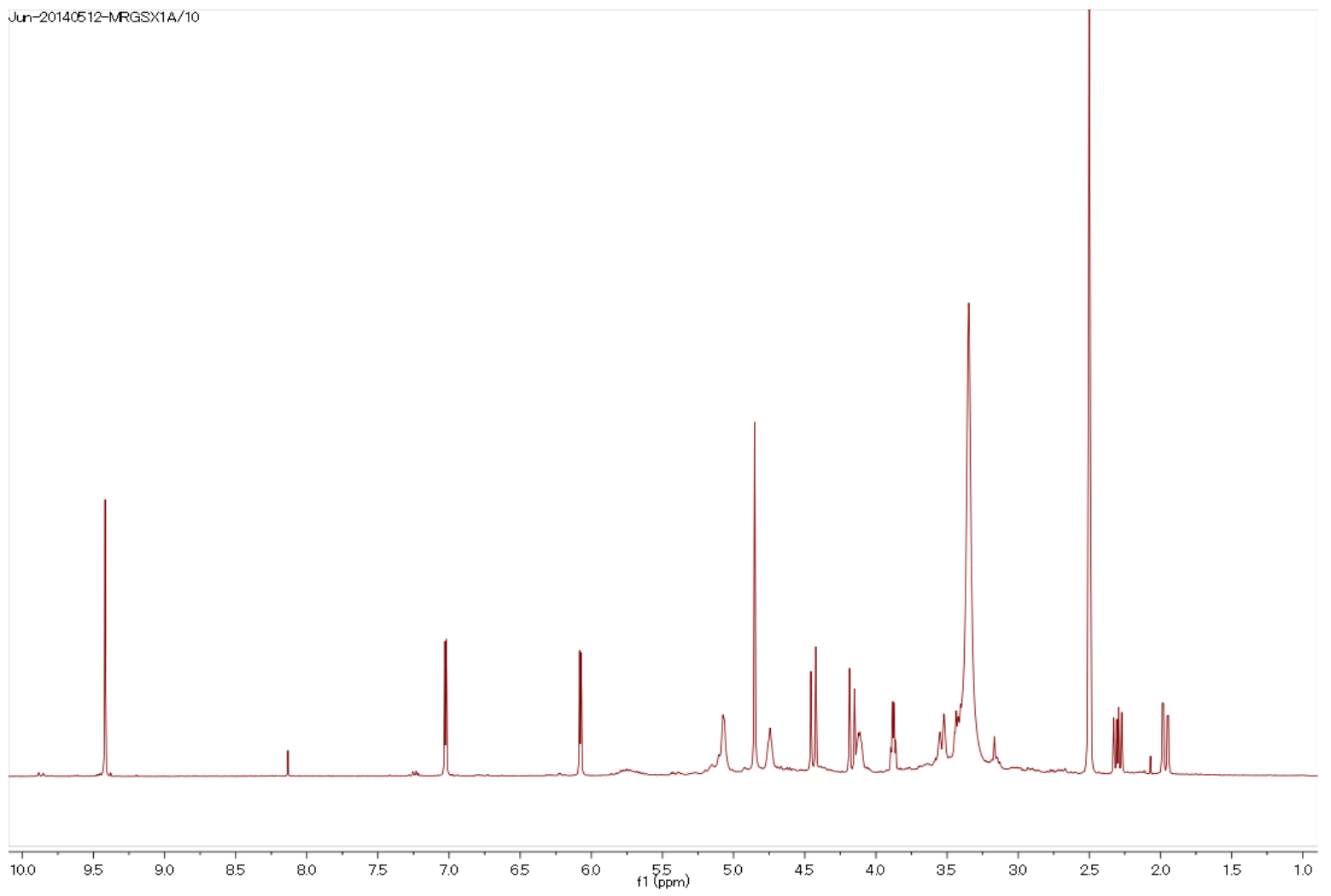

Figure S3. ${ }^{1} \mathrm{H}$ NMR spectrum (DMSO- $d_{6}, 400 \mathrm{MHz}$ )

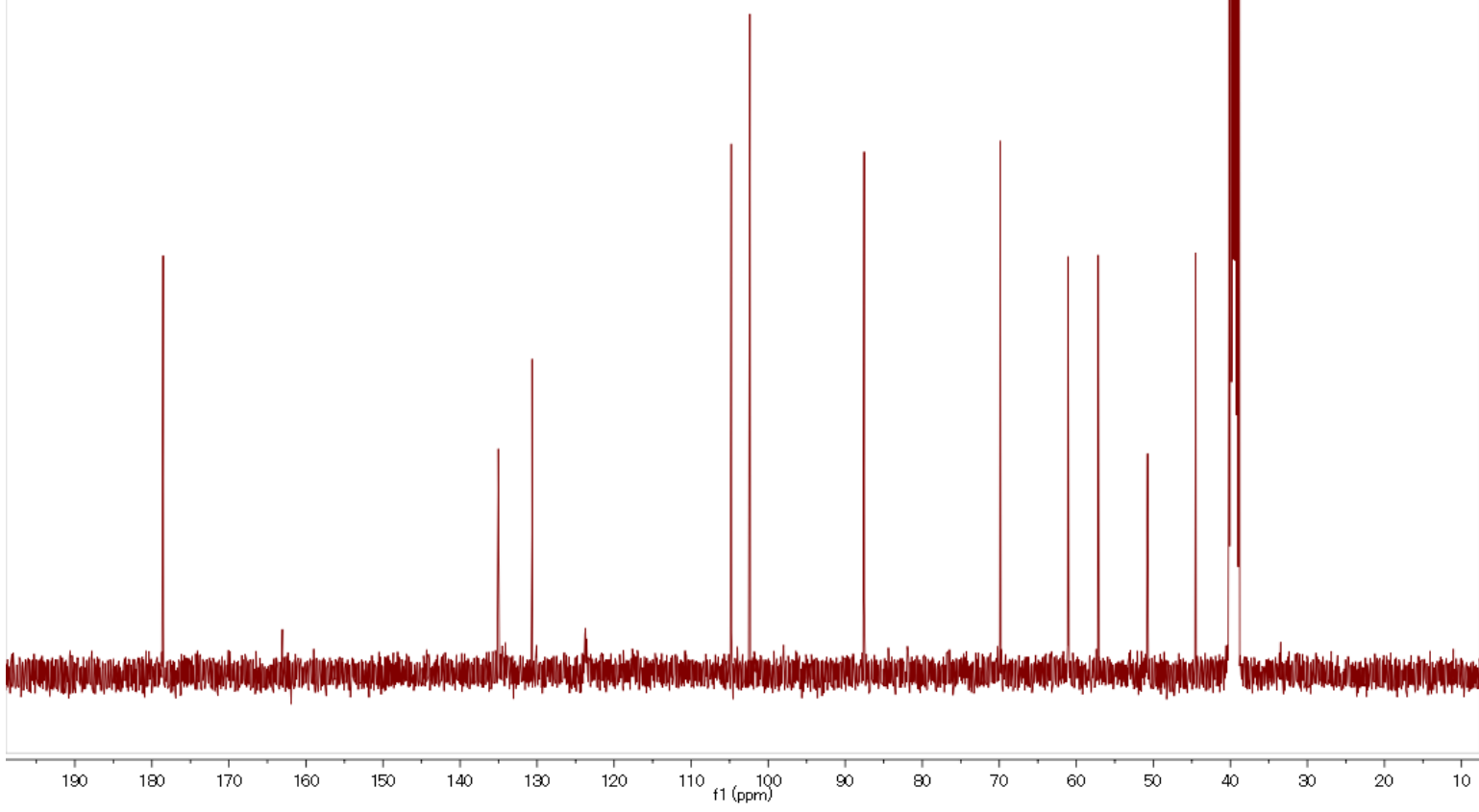

Figure $S 4 .{ }^{13} \mathrm{C}$ NMR spectrum (DMSO- $d_{6}, 100 \mathrm{MHz}$ ) 


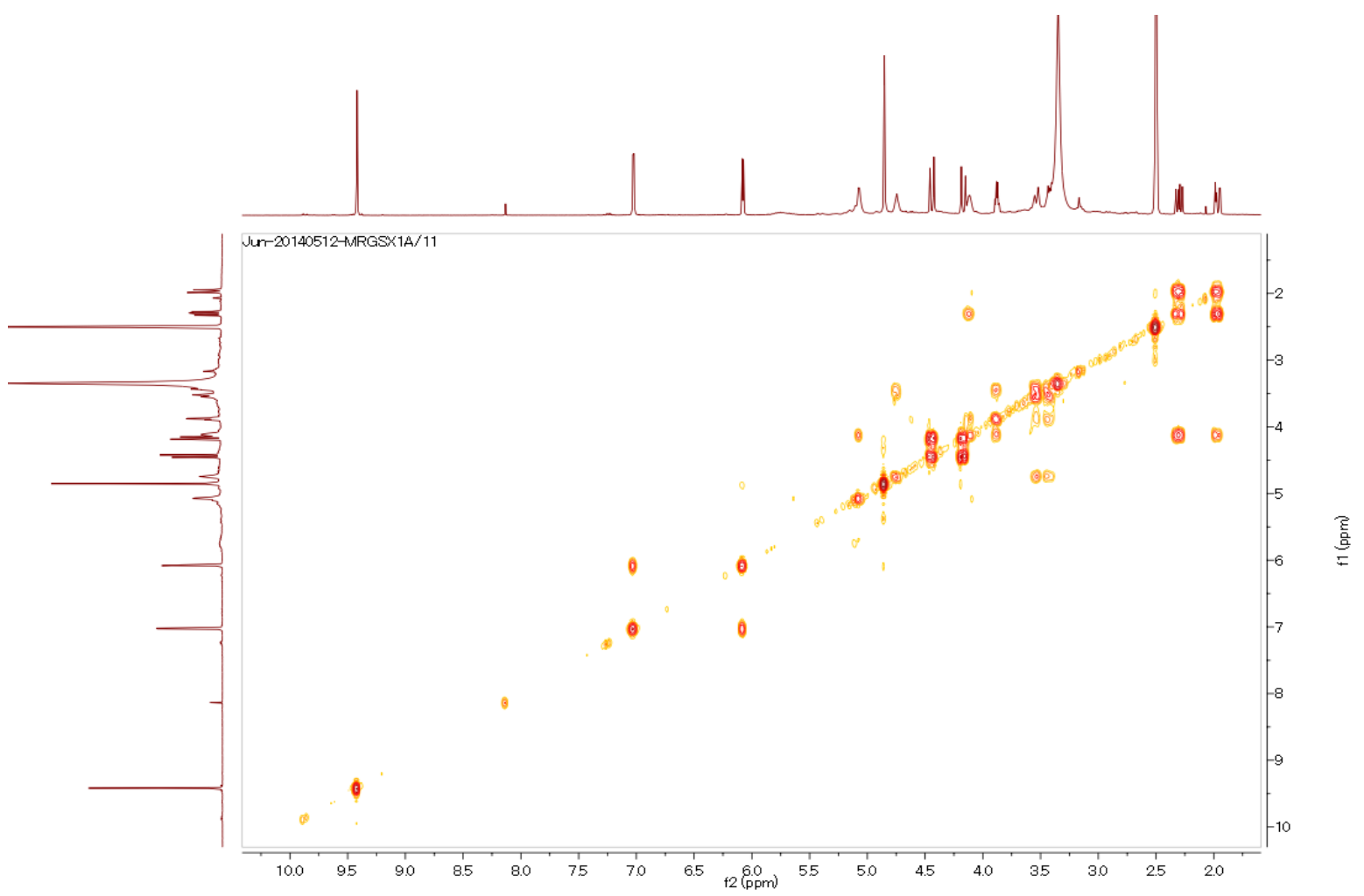

Figure S5. COSY correlations

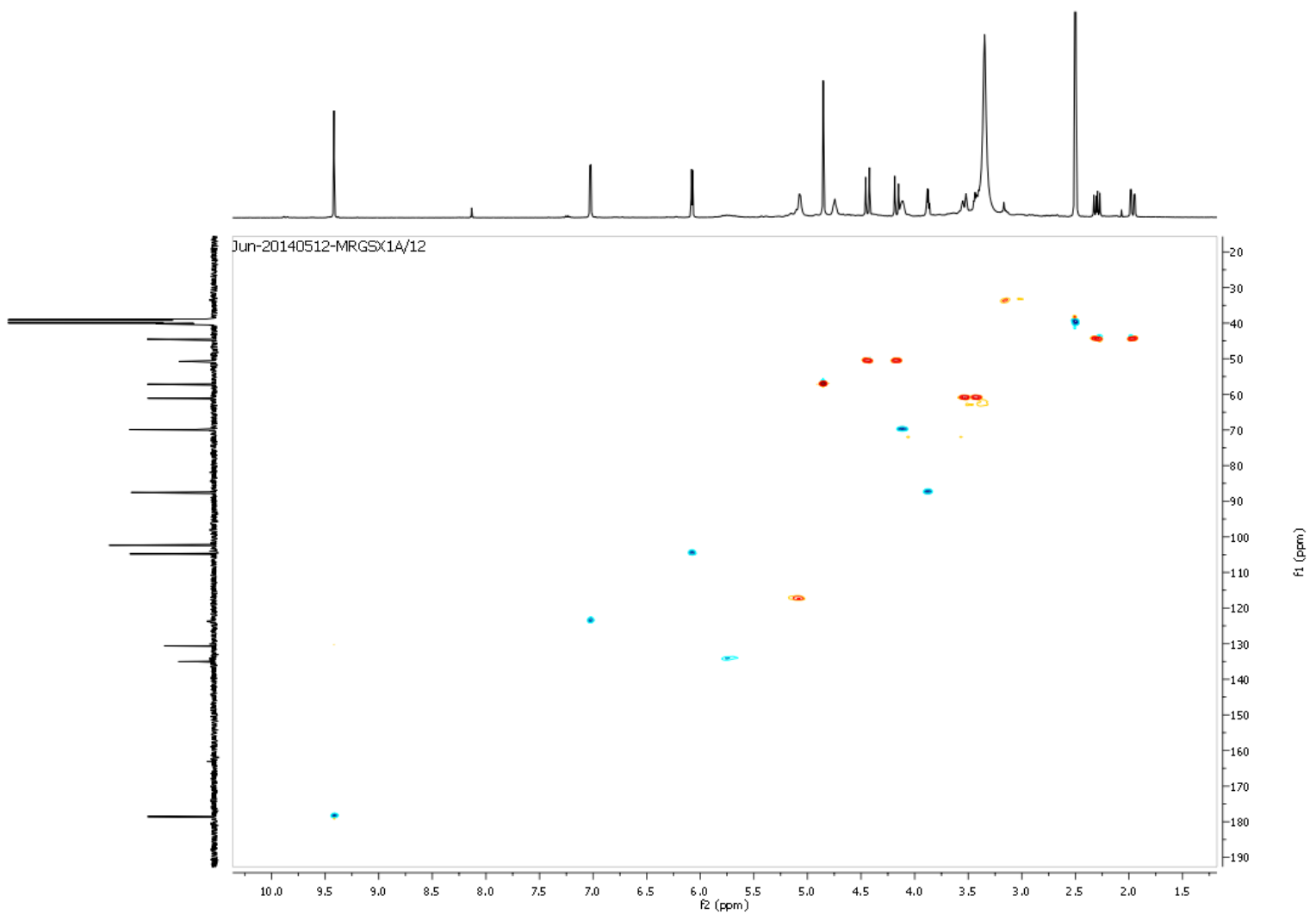

Figure S6. HSQC correlations 


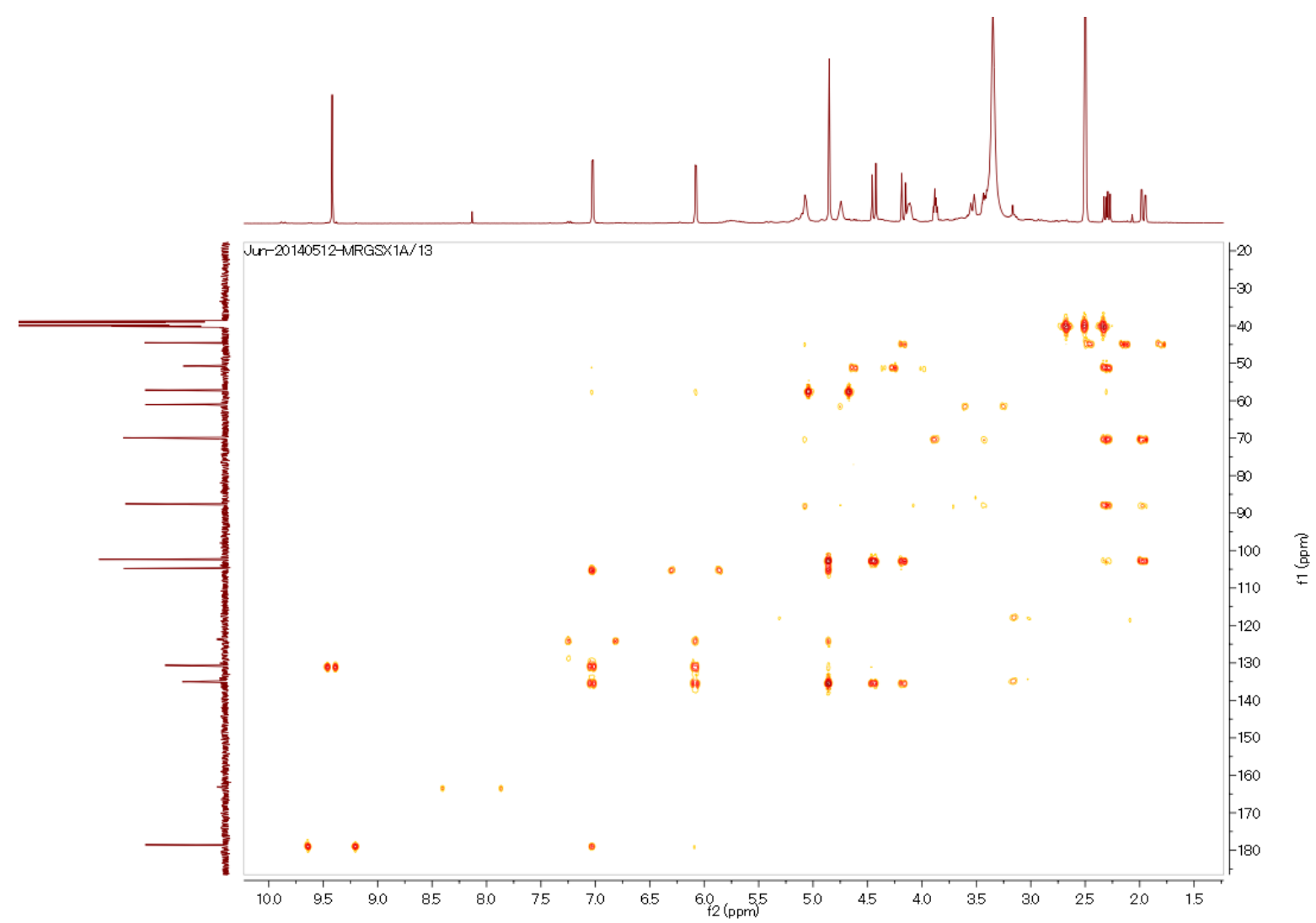

Figure S7. HMBC correlations

\section{Pollenopyrroside A (2)}

Jun

20150508 Acor X1 518 (2.035) Cm (517:521)

100

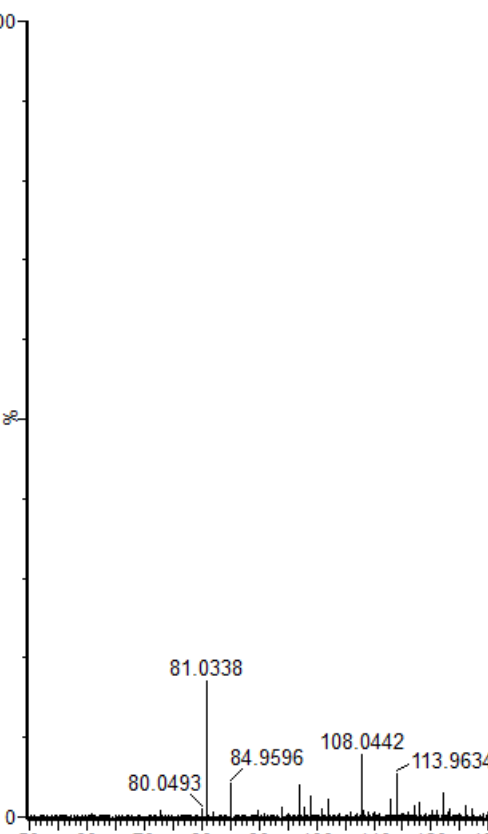

254.1021

1: TOF MS ES+ $-4 .+2$
$4.51 e 4$

Figure S8. HRESIMS (positive) spectra 


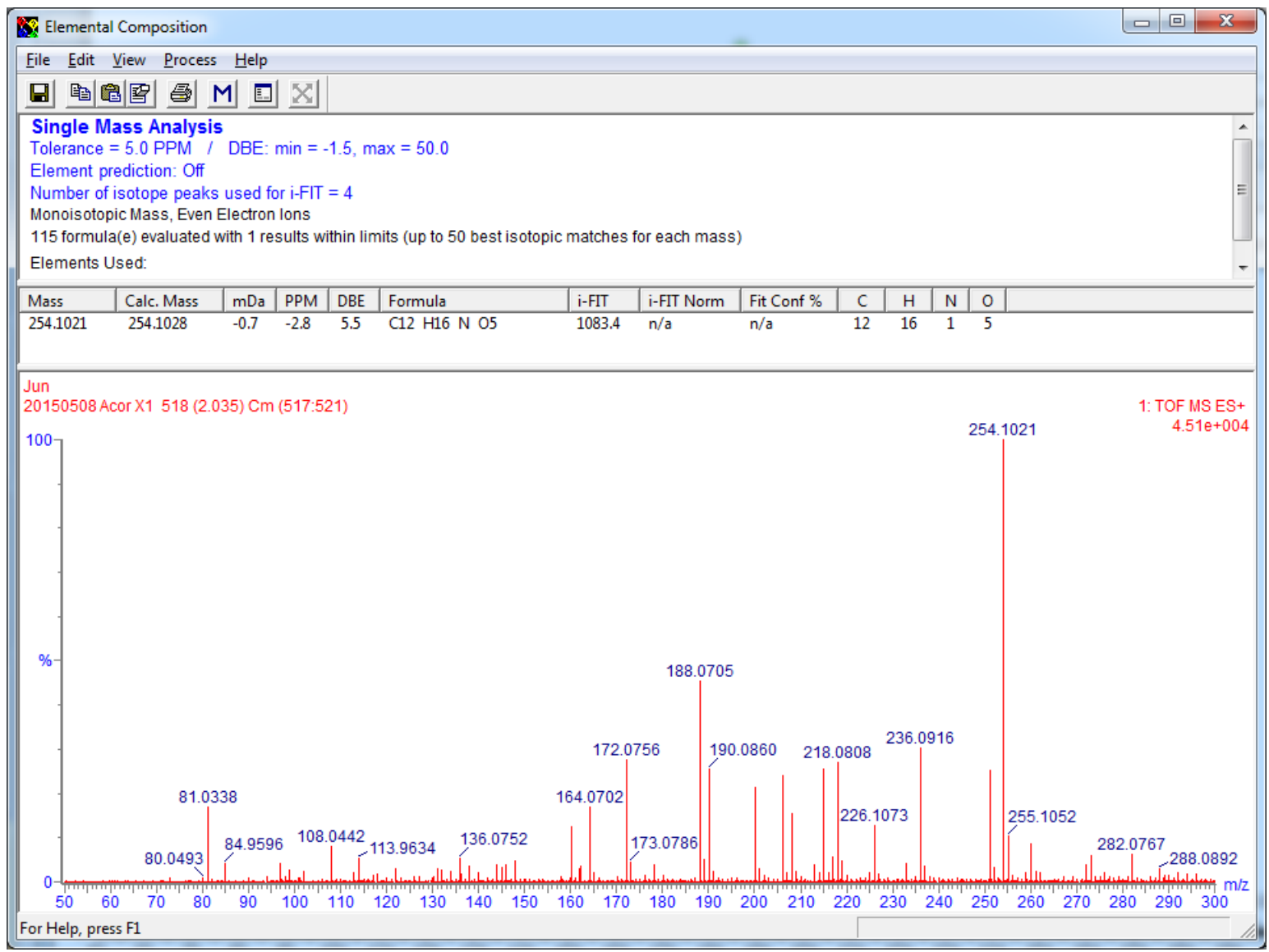

Figure S9. Elemental composition report of the precursor ion

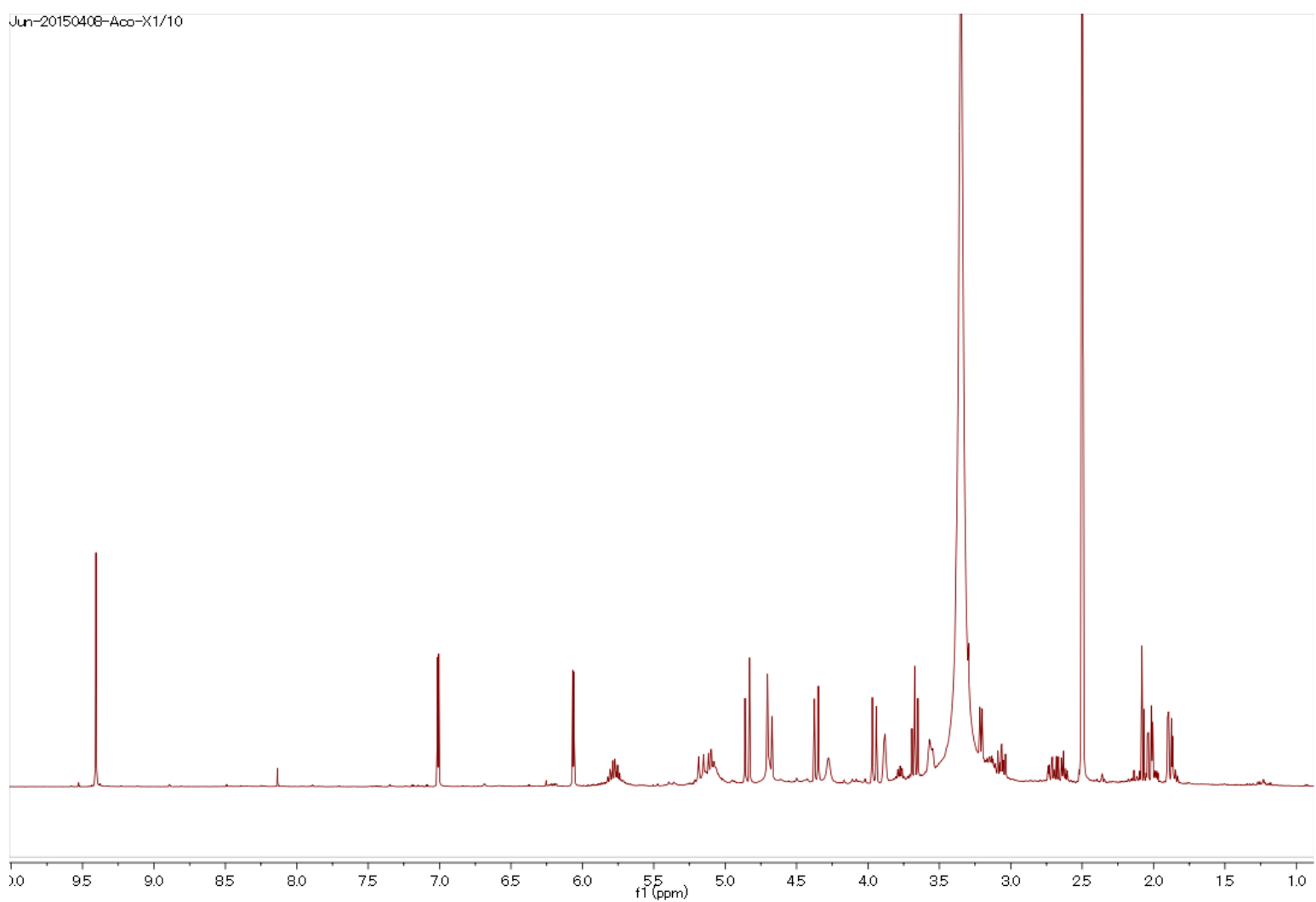

Figure $510 .{ }^{1} \mathrm{H}$ NMR spectrum (DMSO- $d_{6}, 500 \mathrm{MHz}$ ) 


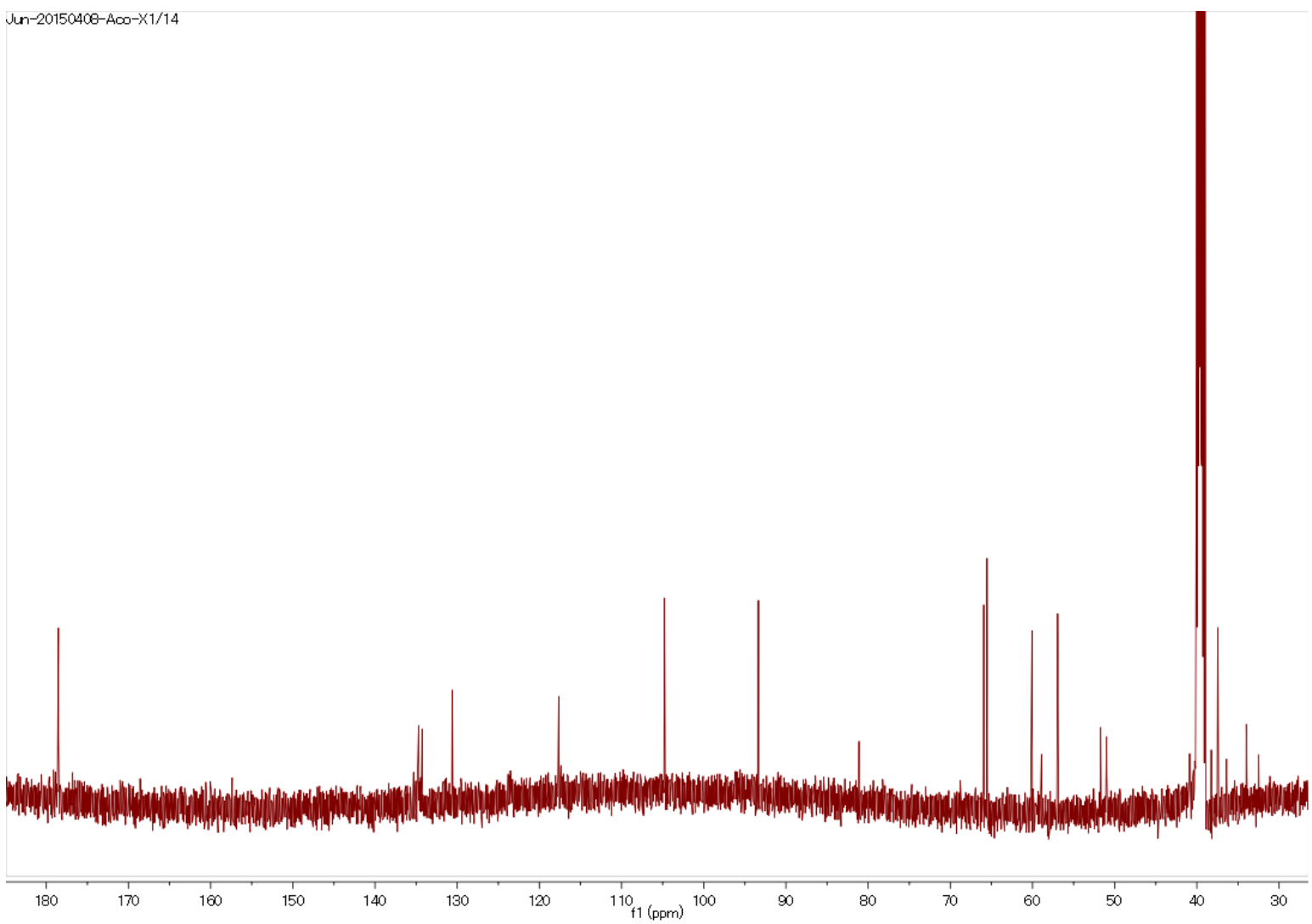

Figure $S 11 .{ }^{13} \mathrm{C}$ NMR spectrum (DMSO- $d_{6}, 125 \mathrm{MHz}$ )

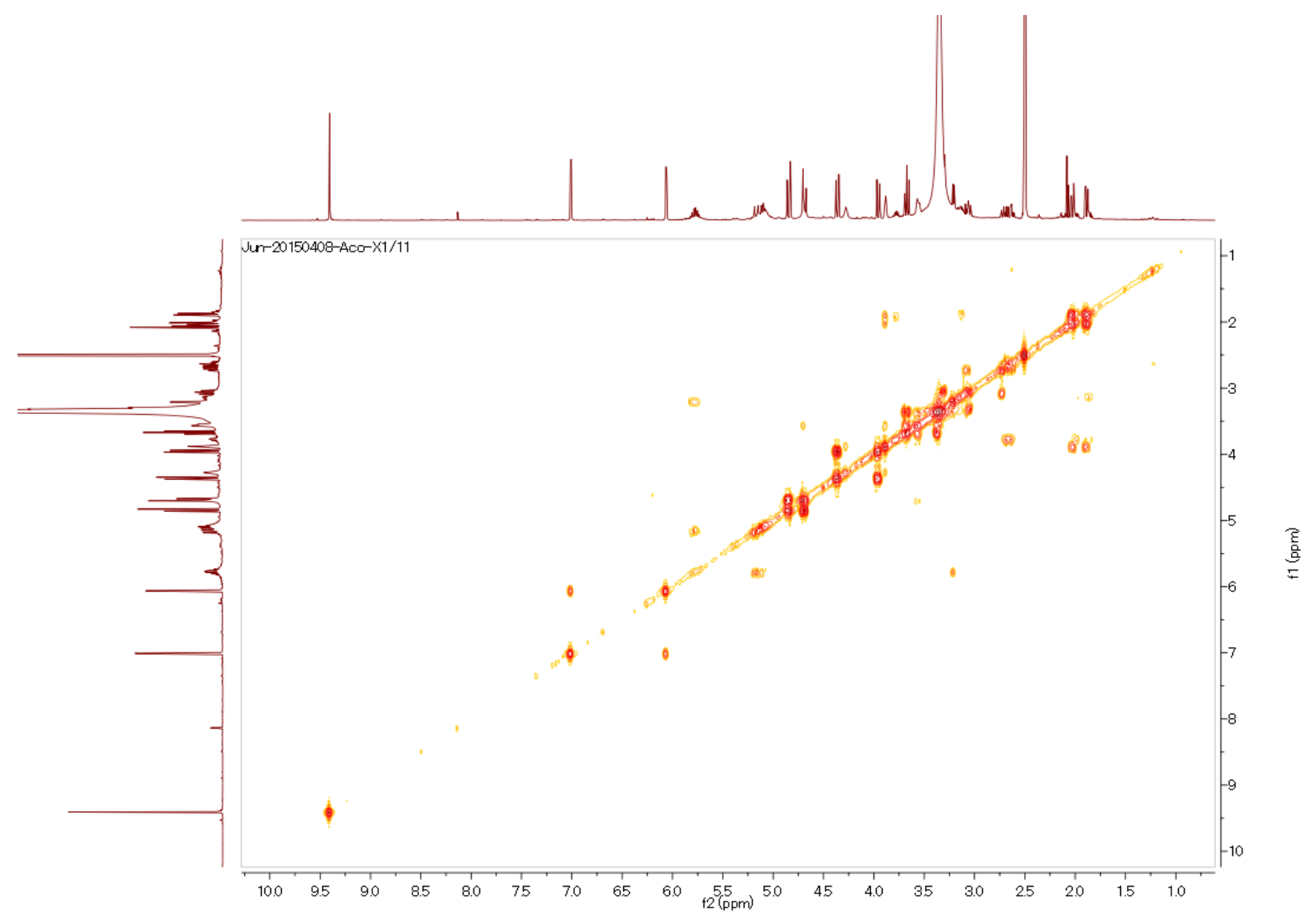

Figure S12. COSY correlations 


$$
I
$$




\section{epi-Acortatarin A (3)}

Jun

30052015_MRGS X1b 492 (1.931) Cm (490:494)

100

$\begin{array}{rr} & \\ 254.1032 & \text { 1: TOF MS ES+ } \\ 2.02 \mathrm{e} 6\end{array}$

Figure S15. HRESIMS (positive) spectrum

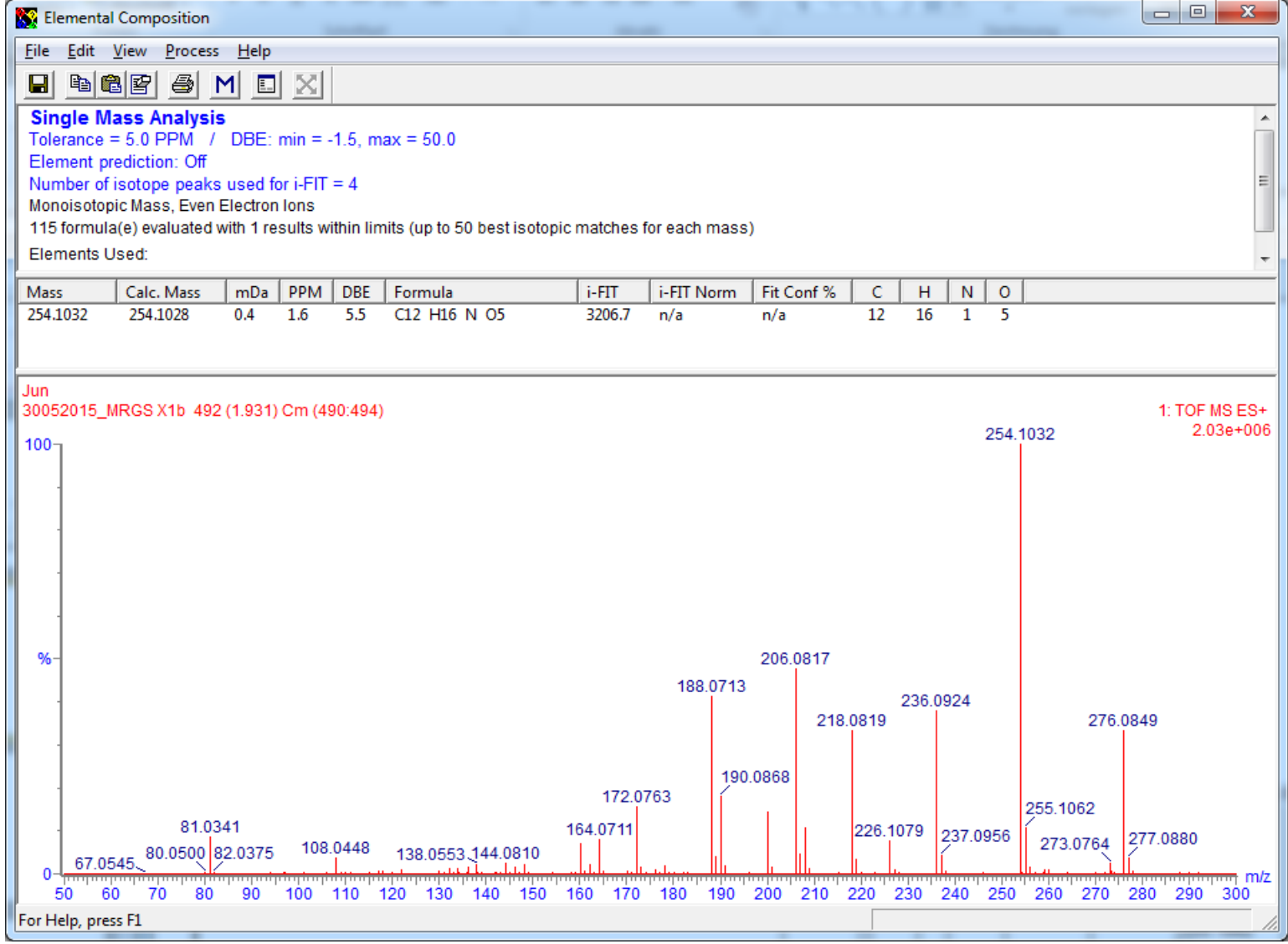

Figure S16. Elemental composition report of the precursor ion 


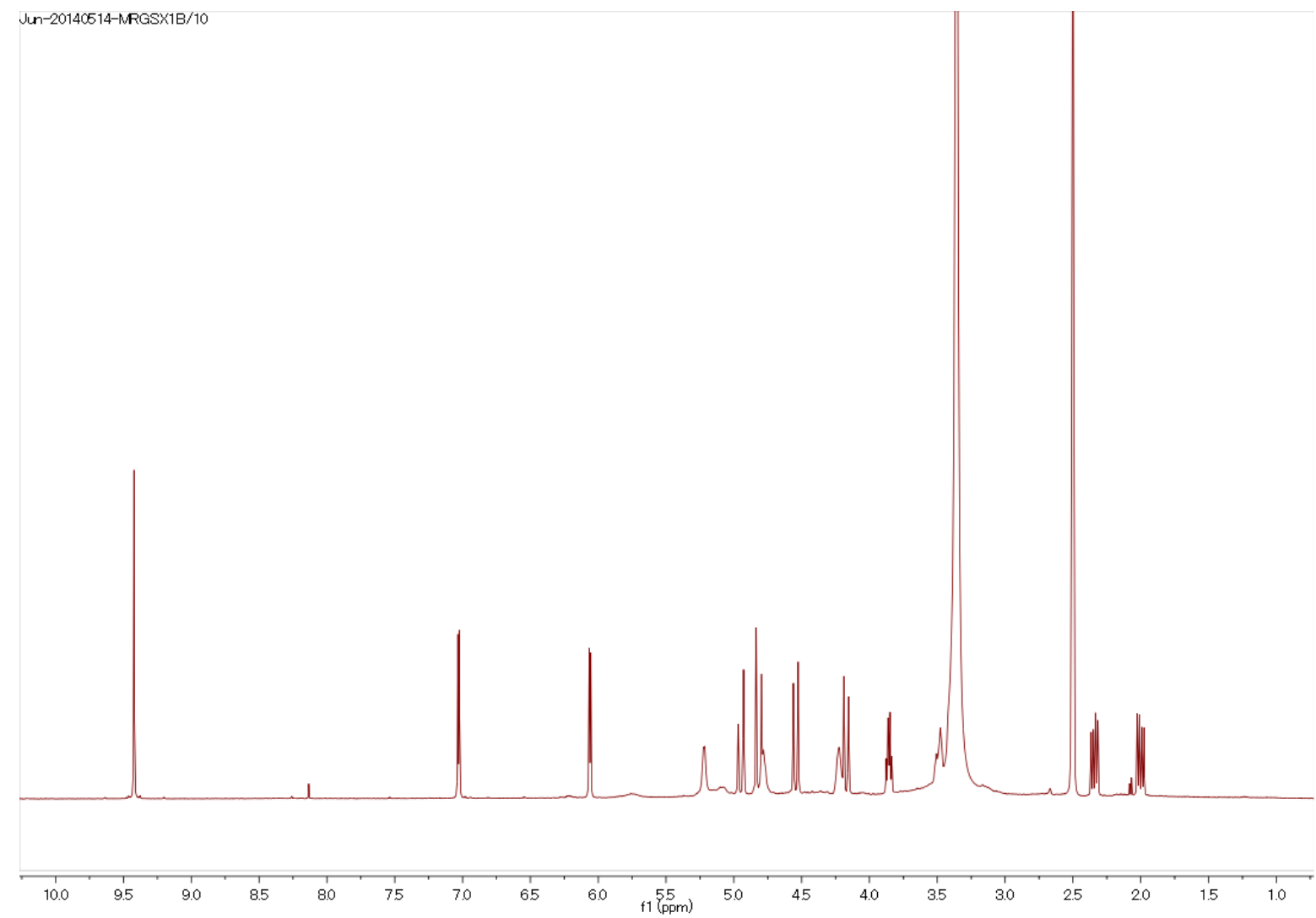

Figure S17. ${ }^{1} \mathrm{H}$ NMR spectrum (DMSO- $d_{6}, 400 \mathrm{MHz}$ )

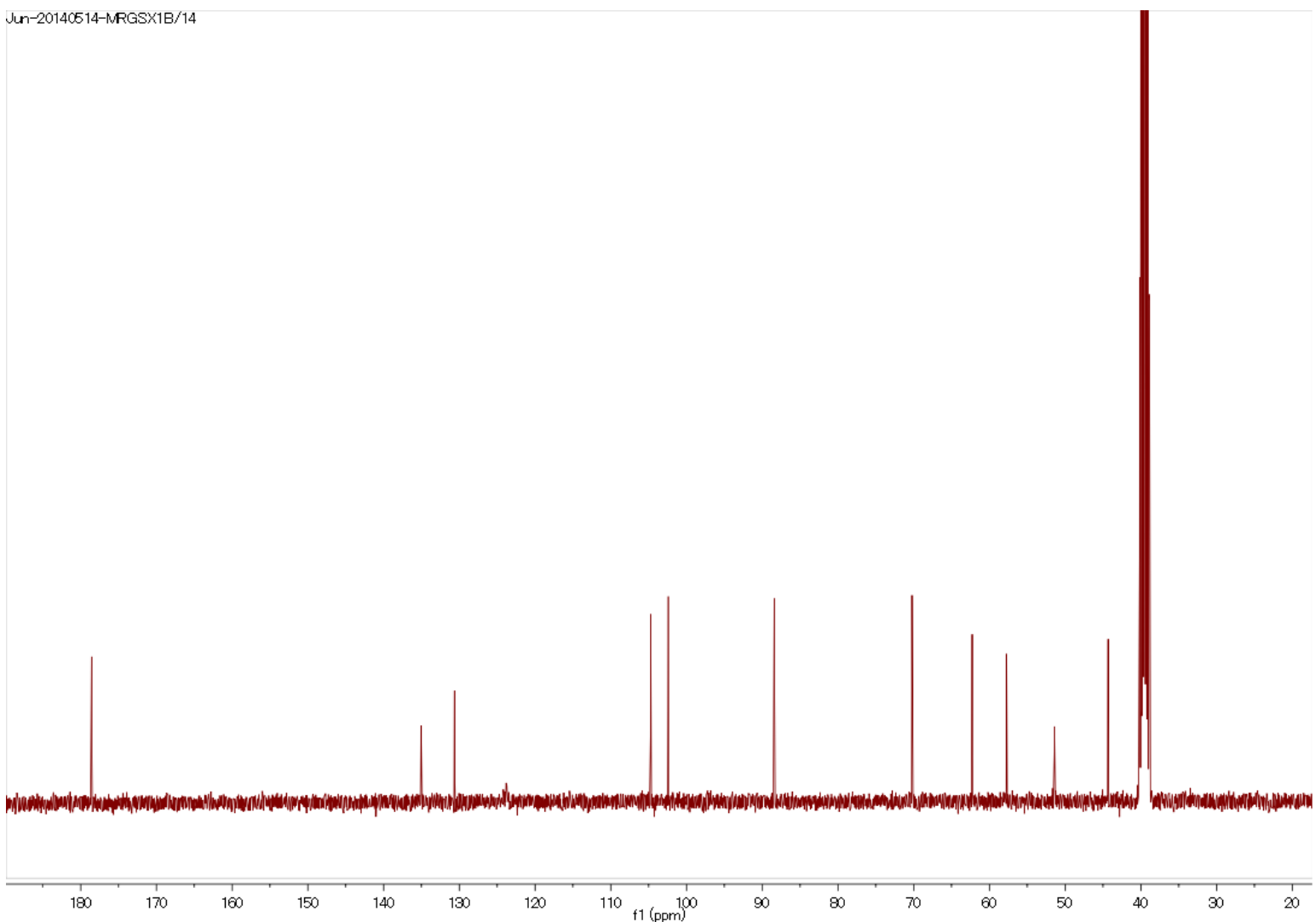

Figure $S 18 .{ }^{13} \mathrm{C}$ NMR spectrum (DMSO- $d_{6}, 100 \mathrm{MHz}$ ) 


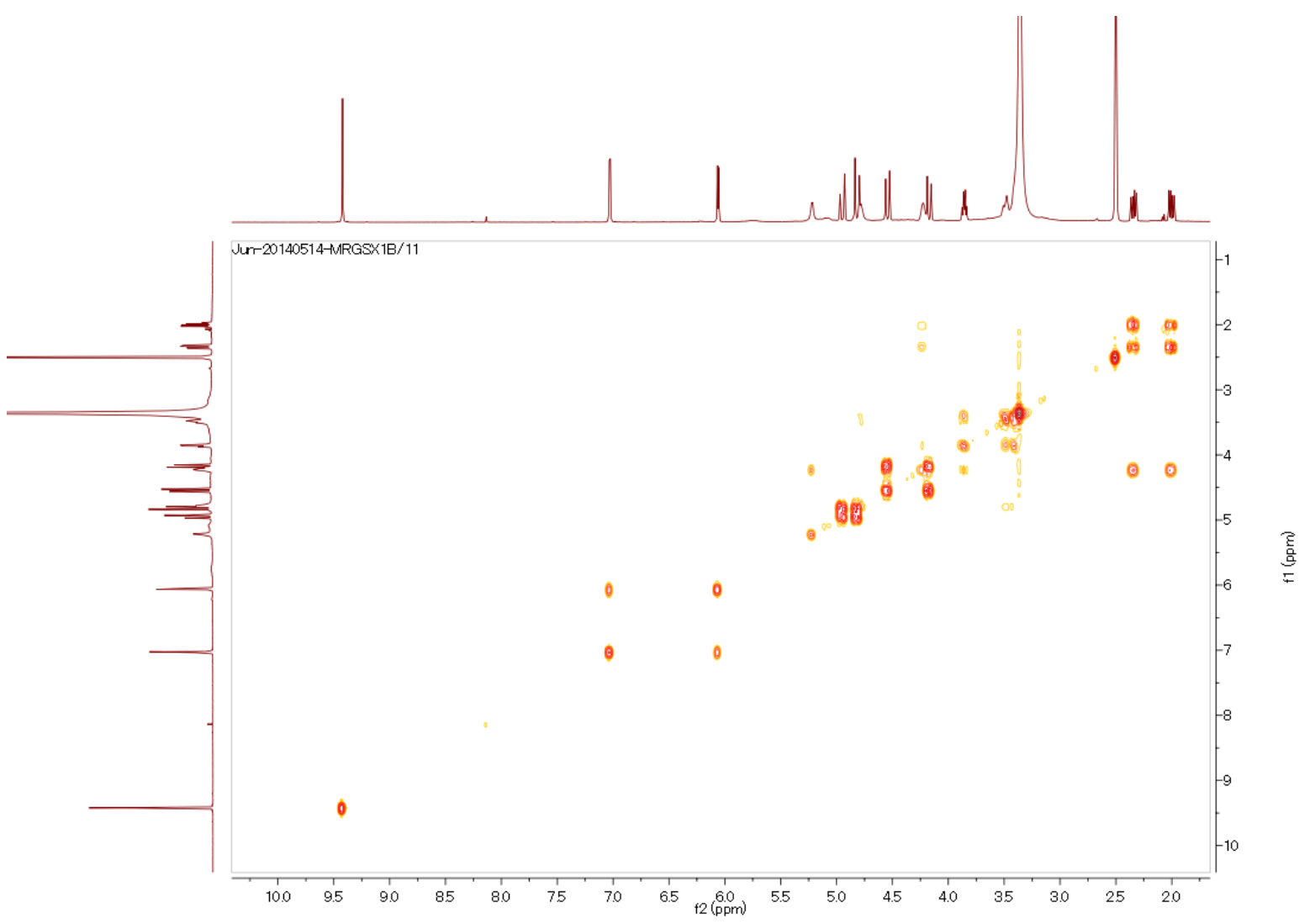

Figure S19. COSY correlations

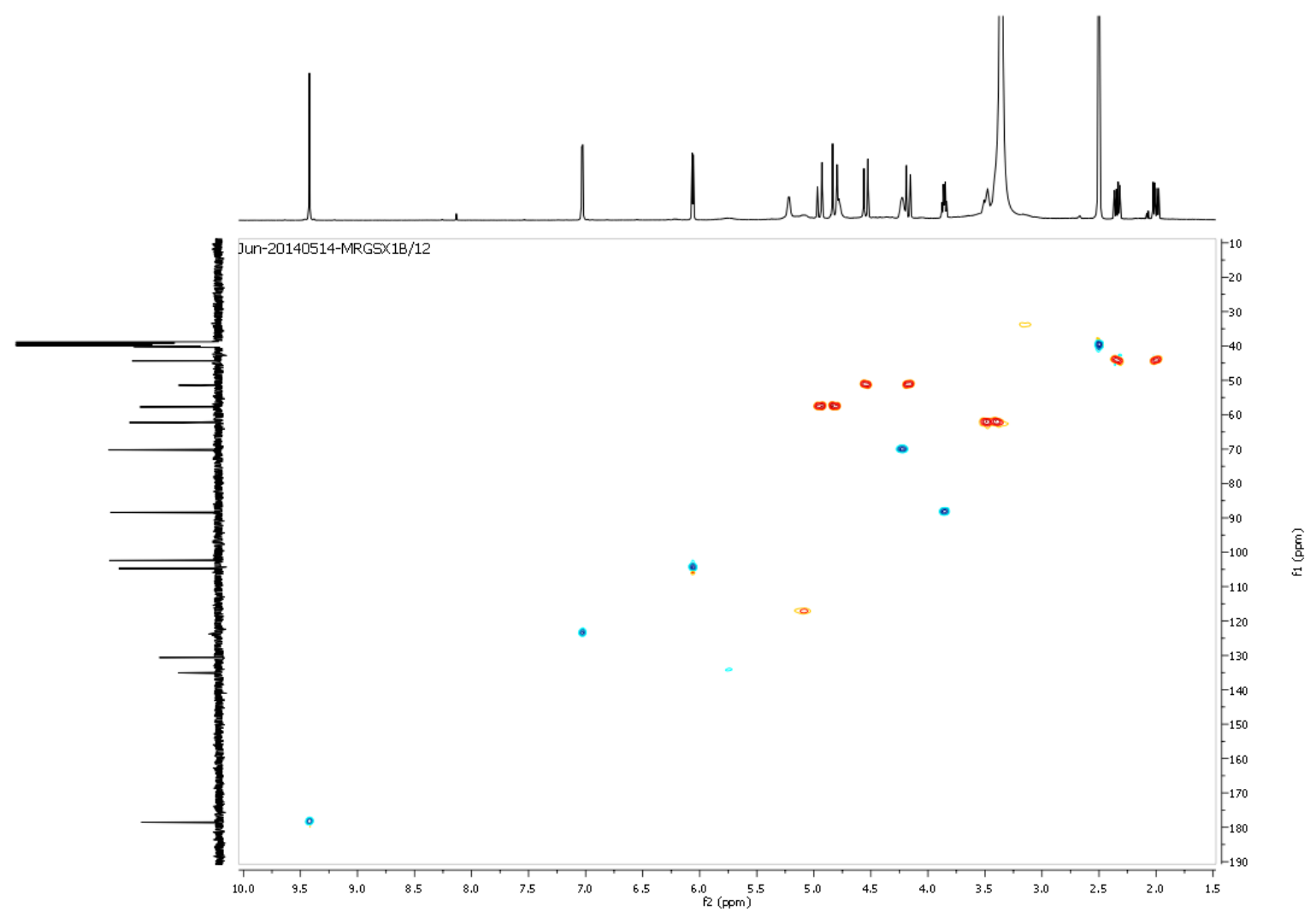

Figure S20. HSQC correlations 


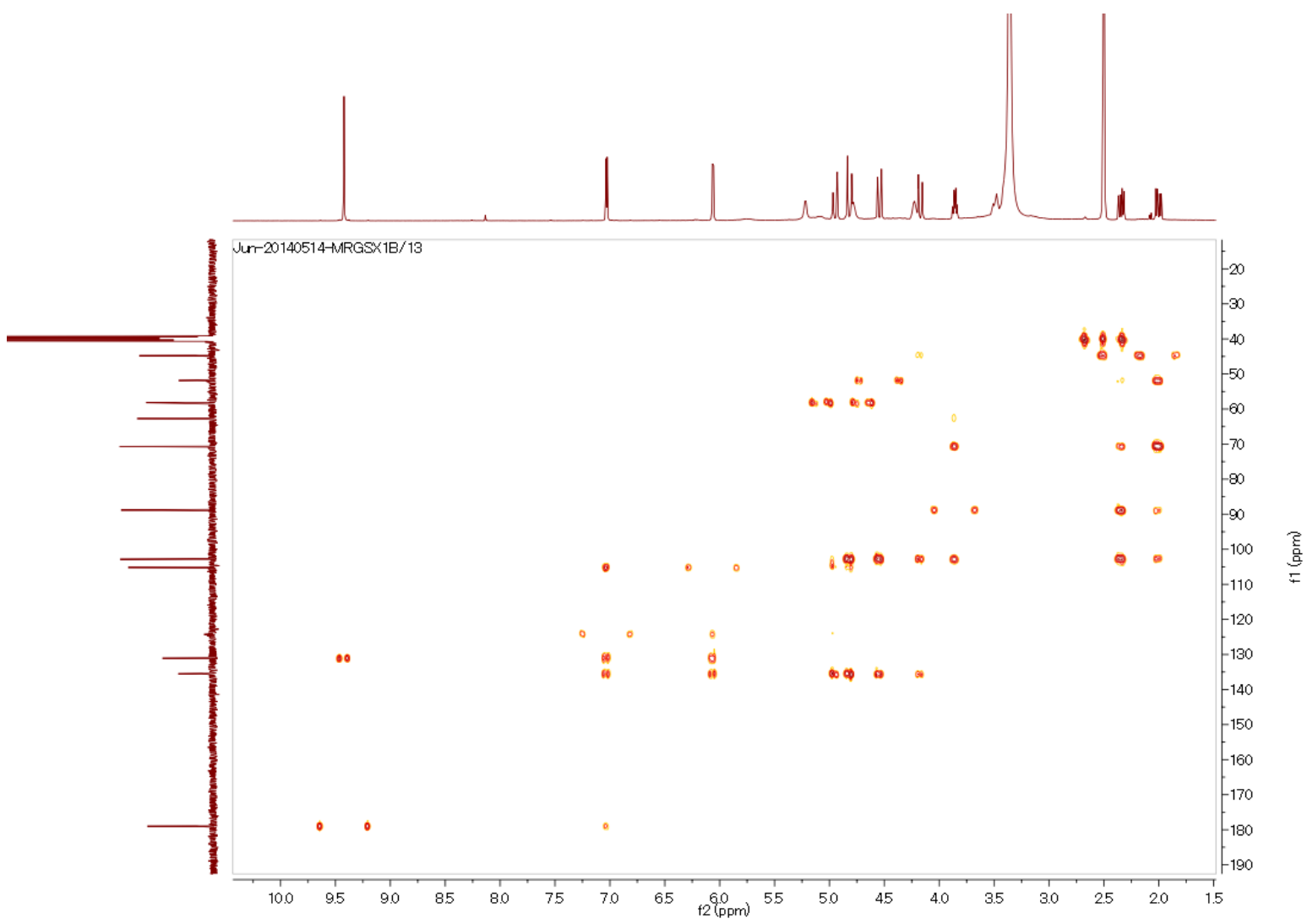

Figure S21. HMBC correlations

\section{Xylapyrroside A (4)}

Jun

20150508 Acor X2 536 (2.103) Cm (536:540)

100

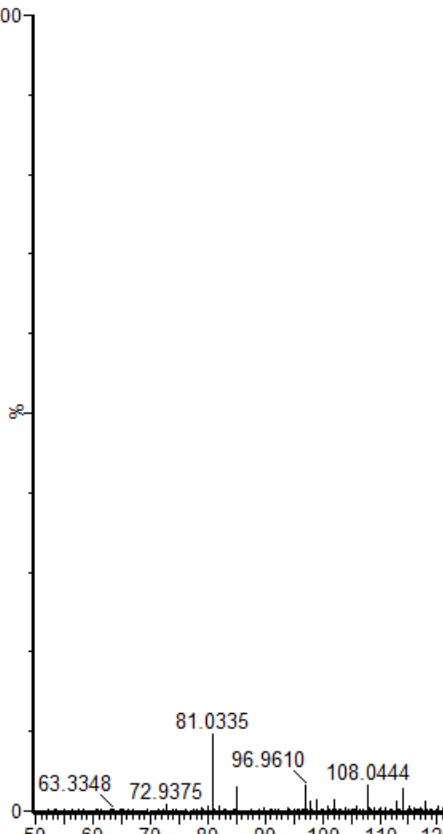

$276.0844^{1:}$ TOF MS ES+ $_{7.34 \mathrm{e}}$

Figure S22. HRESIMS (positive) spectrum 


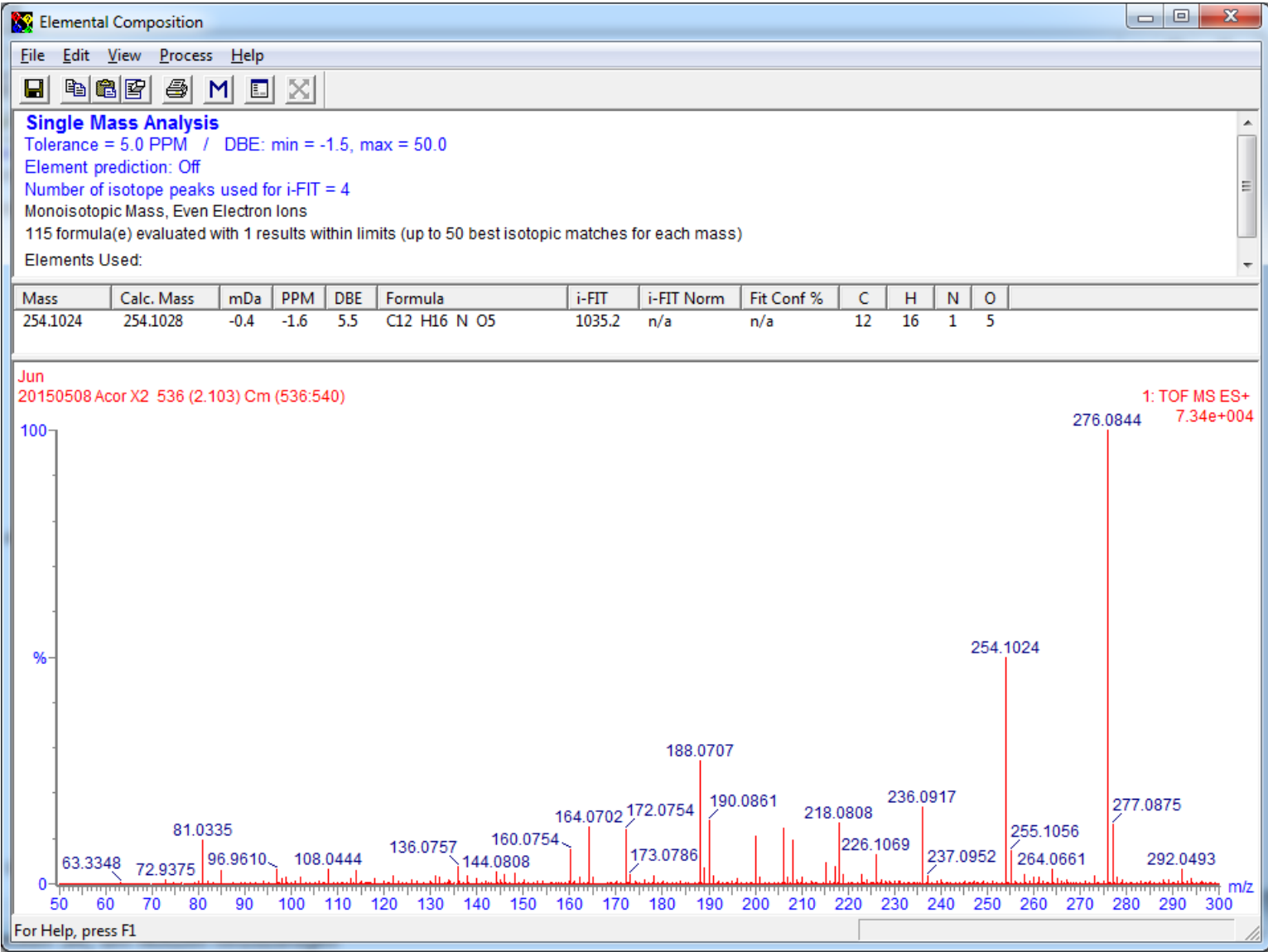

Figure S23. Elemental composition report of the precursor ion 


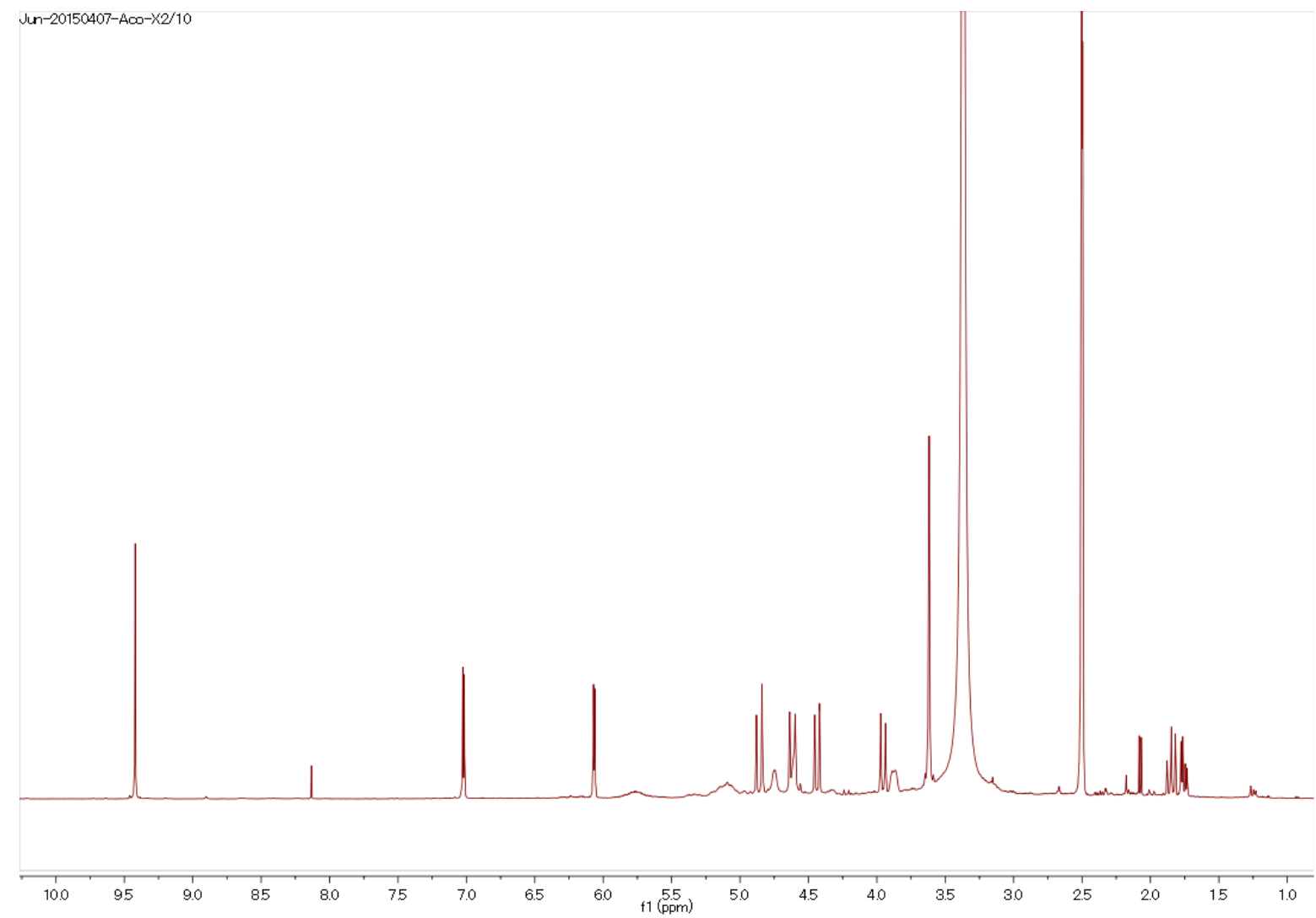

Figure S24. ${ }^{1} \mathrm{H}$ NMR spectrum (DMSO- $d_{6}, 400 \mathrm{MHz}$ )

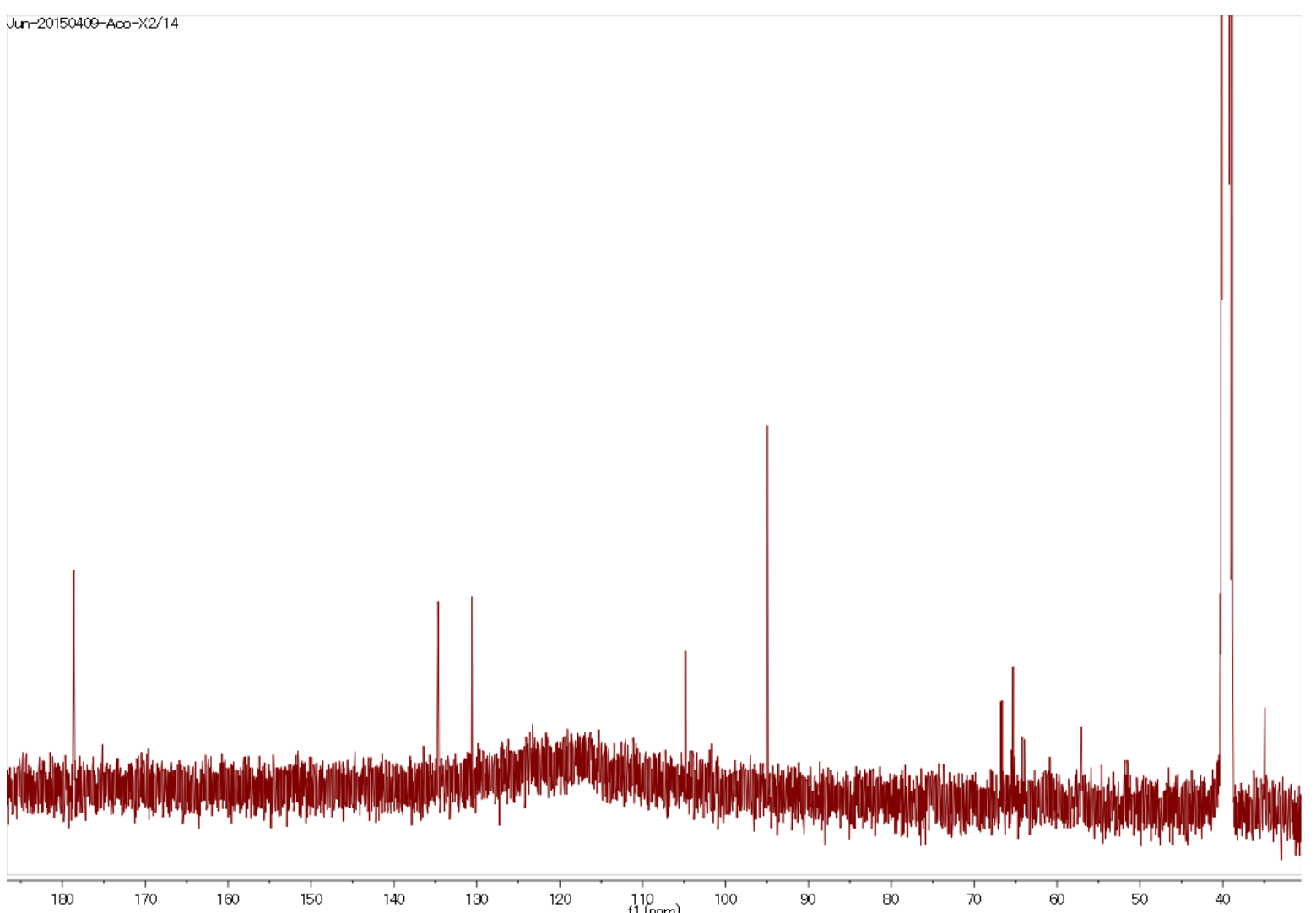

Figure S25. ${ }^{13} \mathrm{C}$ NMR spectrum (DMSO- $d_{6}, 100 \mathrm{MHz}$ ) 


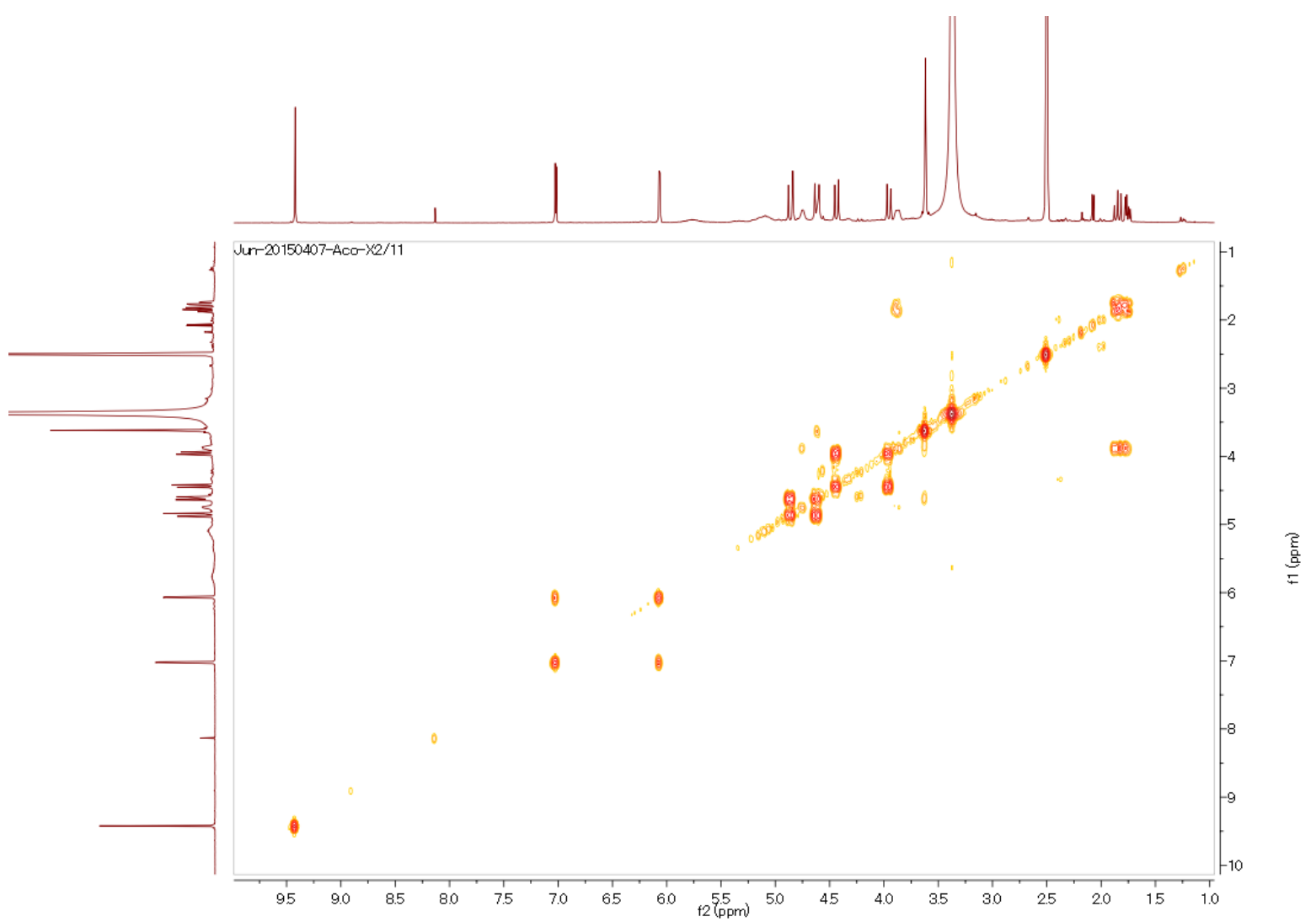

Figure S26. COSY correlations

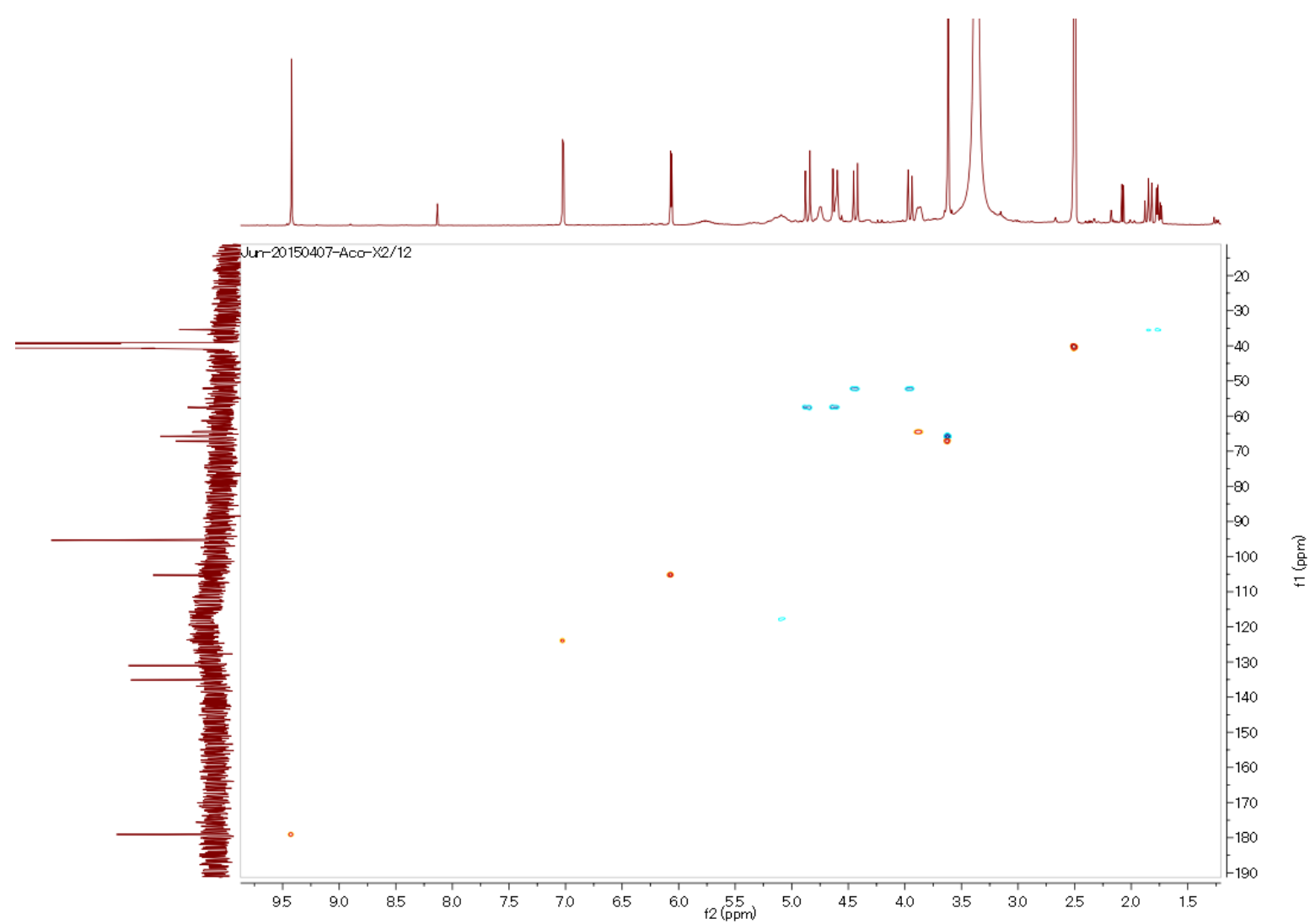

Figure S27. HSQC correlations 


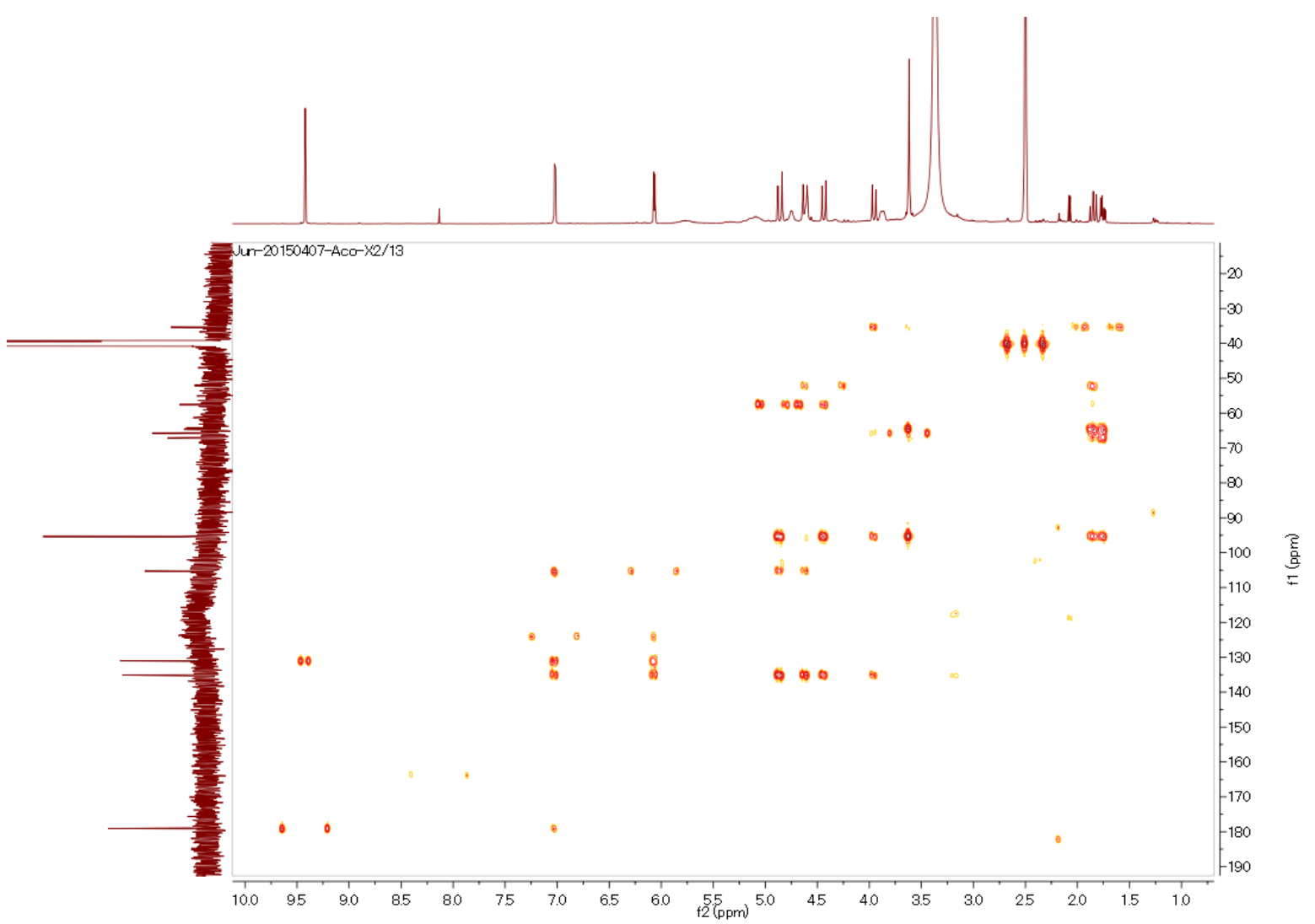

Figure S28. HMBC correlations

$\underline{\text { 5-hydroxymethyl-1-[(5-hydroxymethyl-2-furanyl)methyl]-1H-pyrrole-2-car }}$ baldehyde (5)

Jun
20150508 Acor X3 557 (2.184) Cm (557:563)

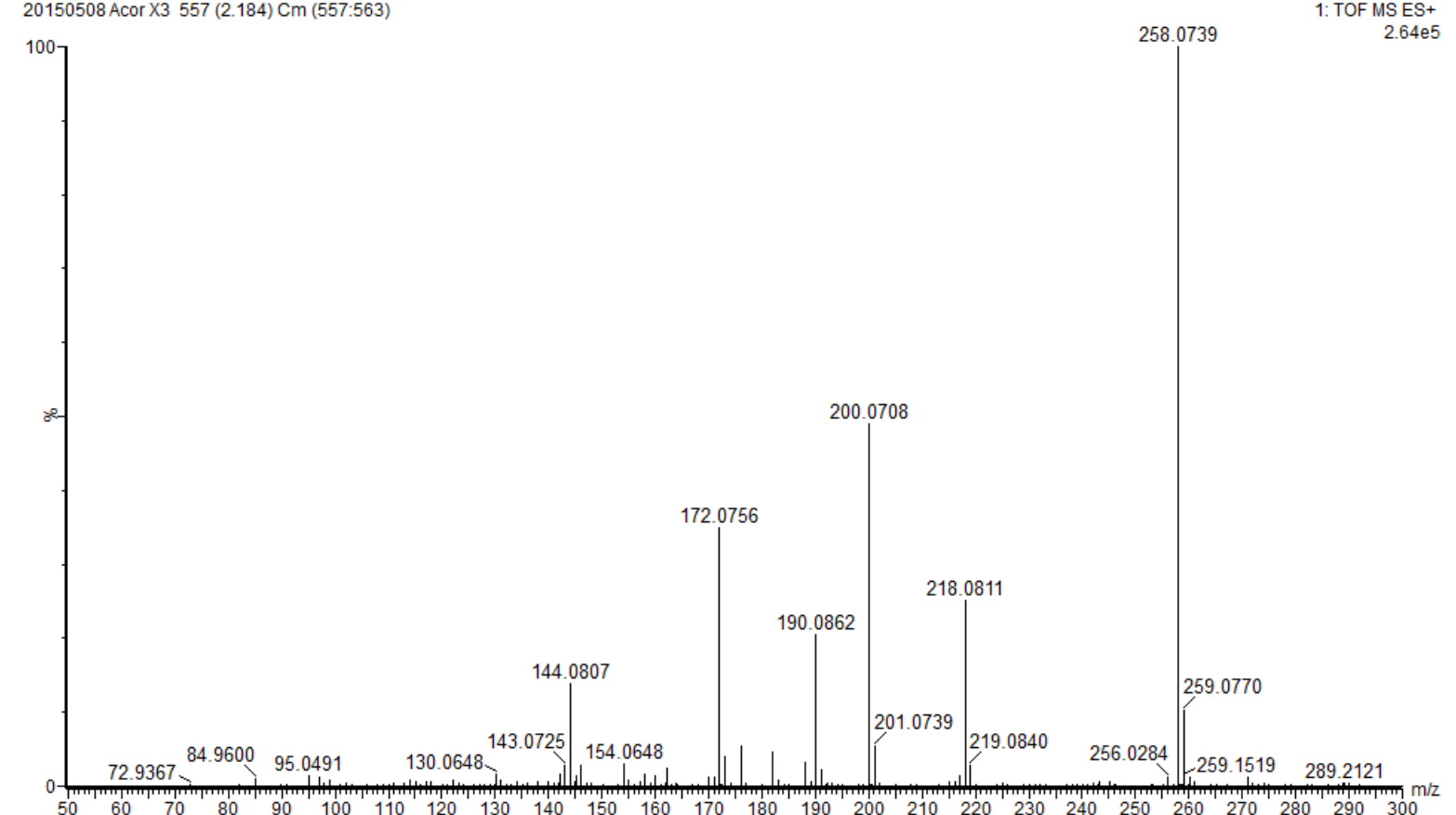

Figure S29. HRESIMS (positive) spectrum 


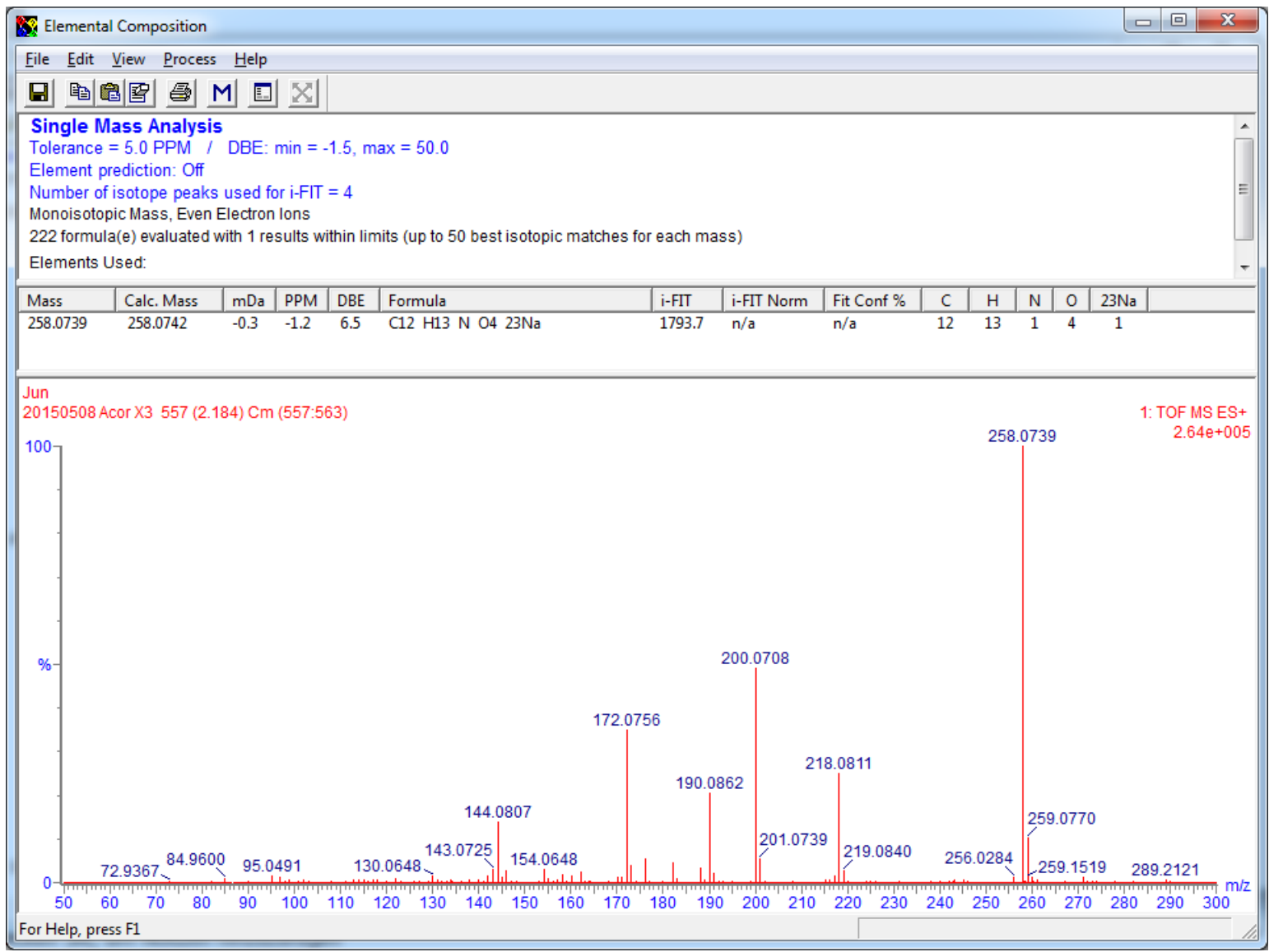

Figure S30. Elemental composition report of the precursor ion

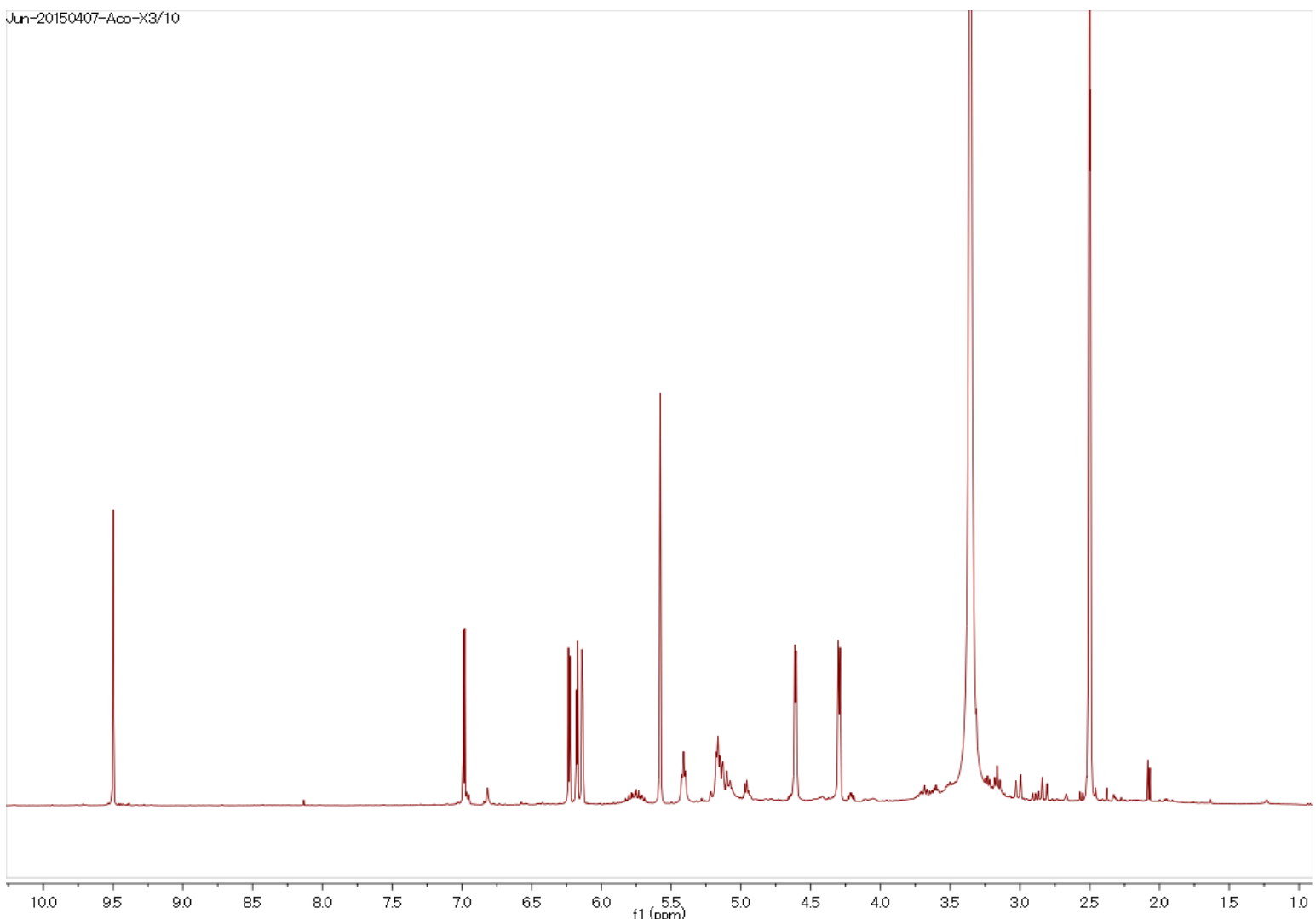

Figure S31. ${ }^{1} \mathrm{H}$ NMR spectrum (DMSO- $d_{6}, 400 \mathrm{MHz}$ ) 


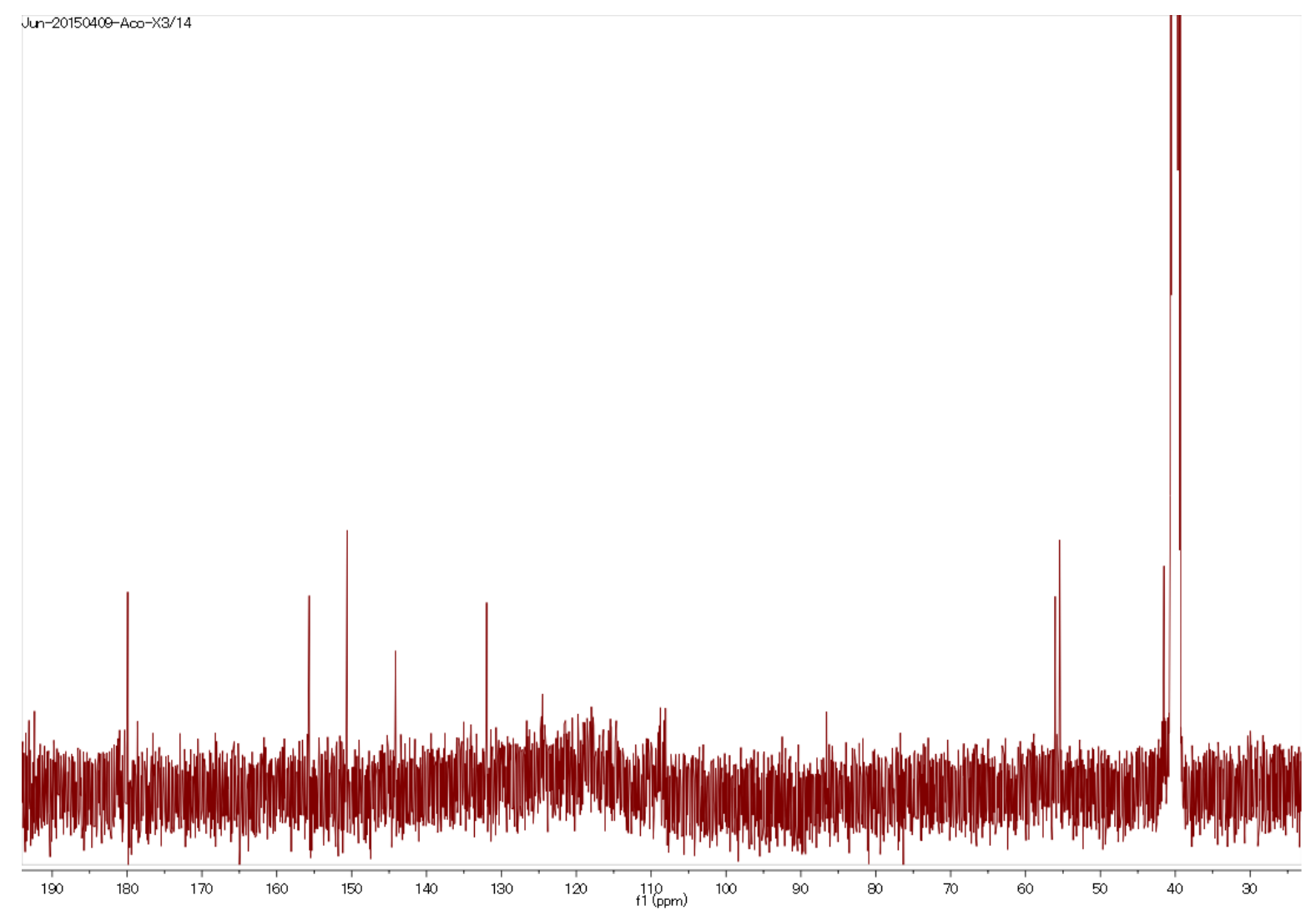

Figure S32. ${ }^{13} \mathrm{C}$ NMR spectrum (DMSO- $d_{6}, 100 \mathrm{MHz}$ )

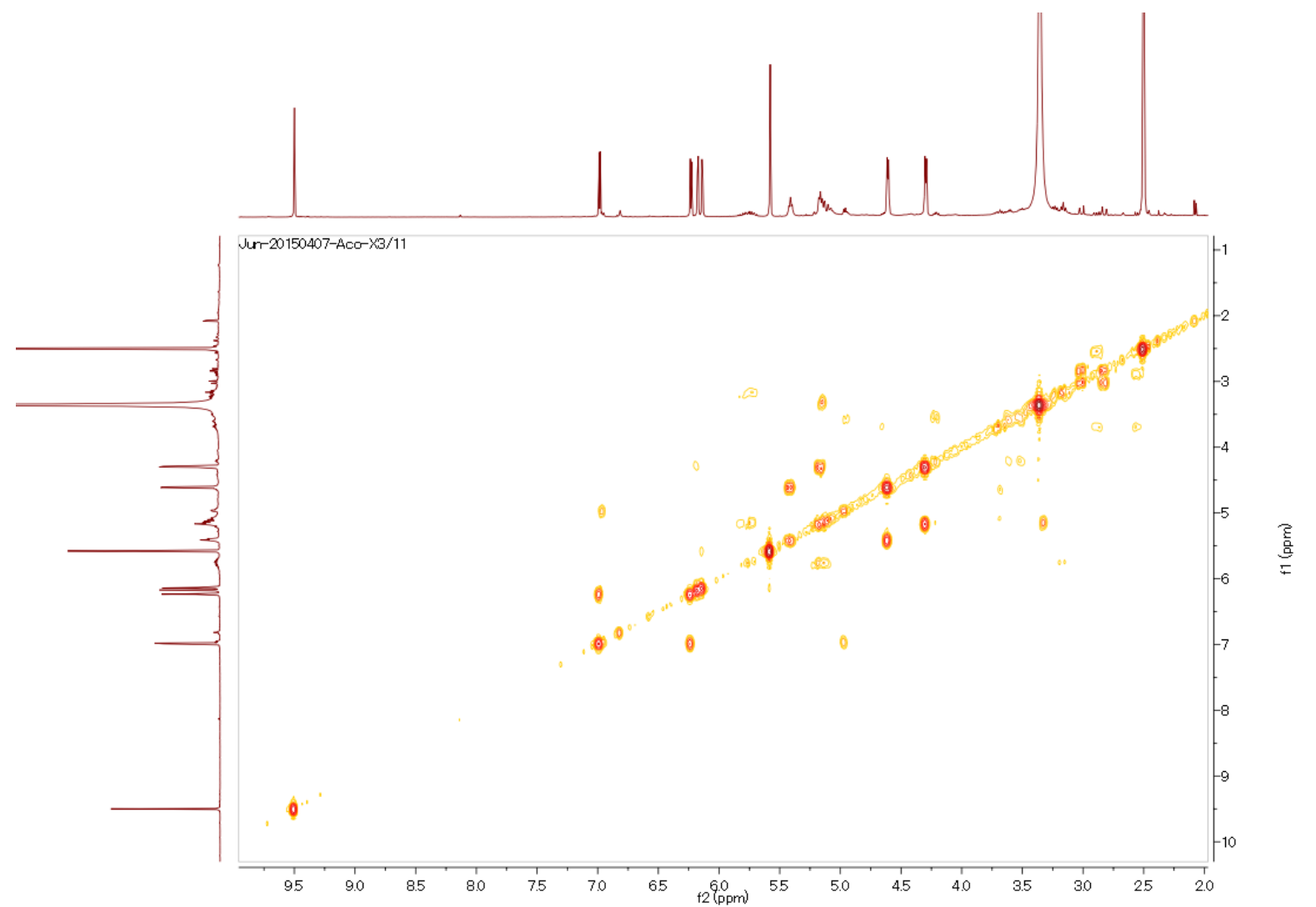

Figure S33. COSY correlations 


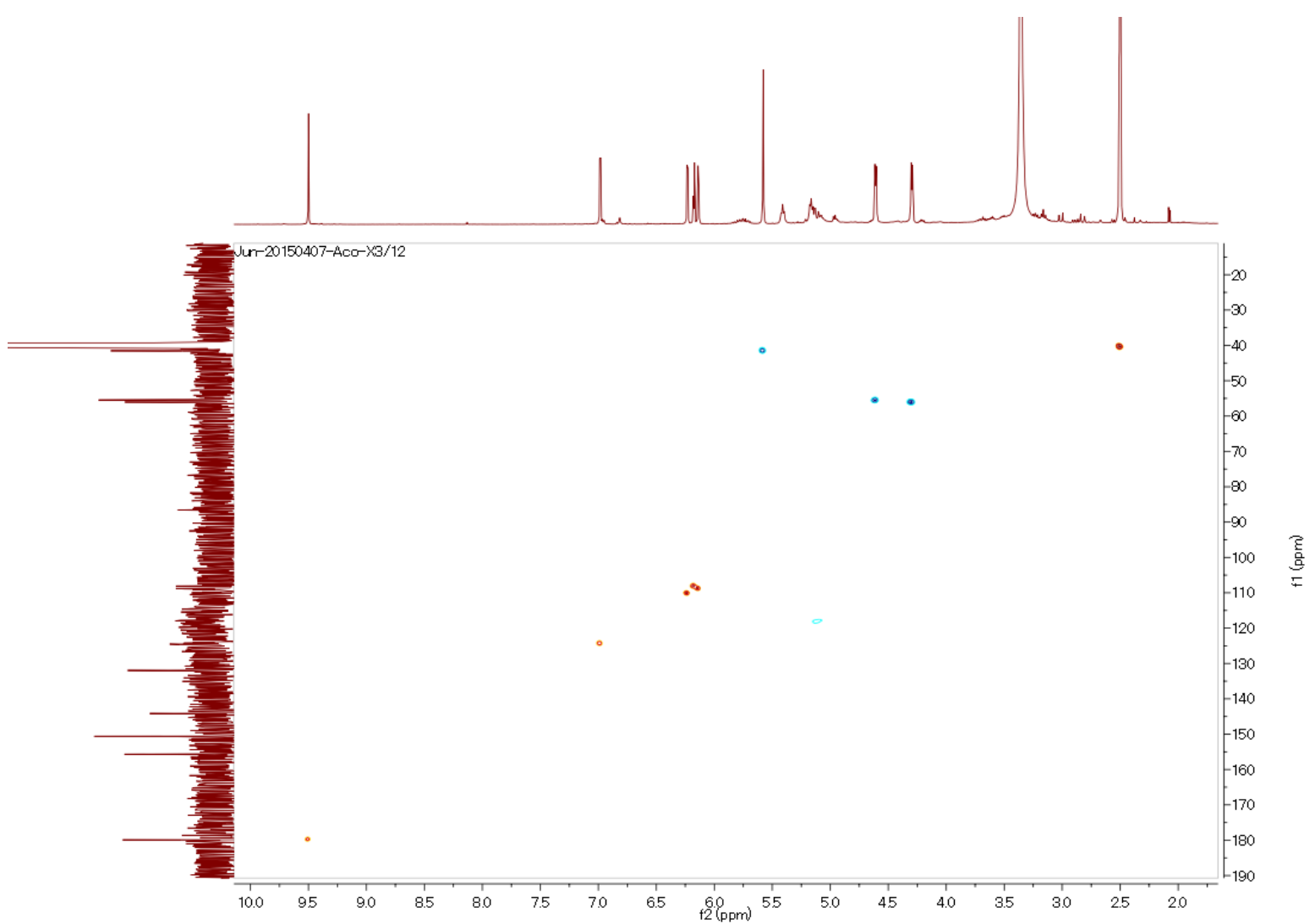

Figure S34. HSQC correlations

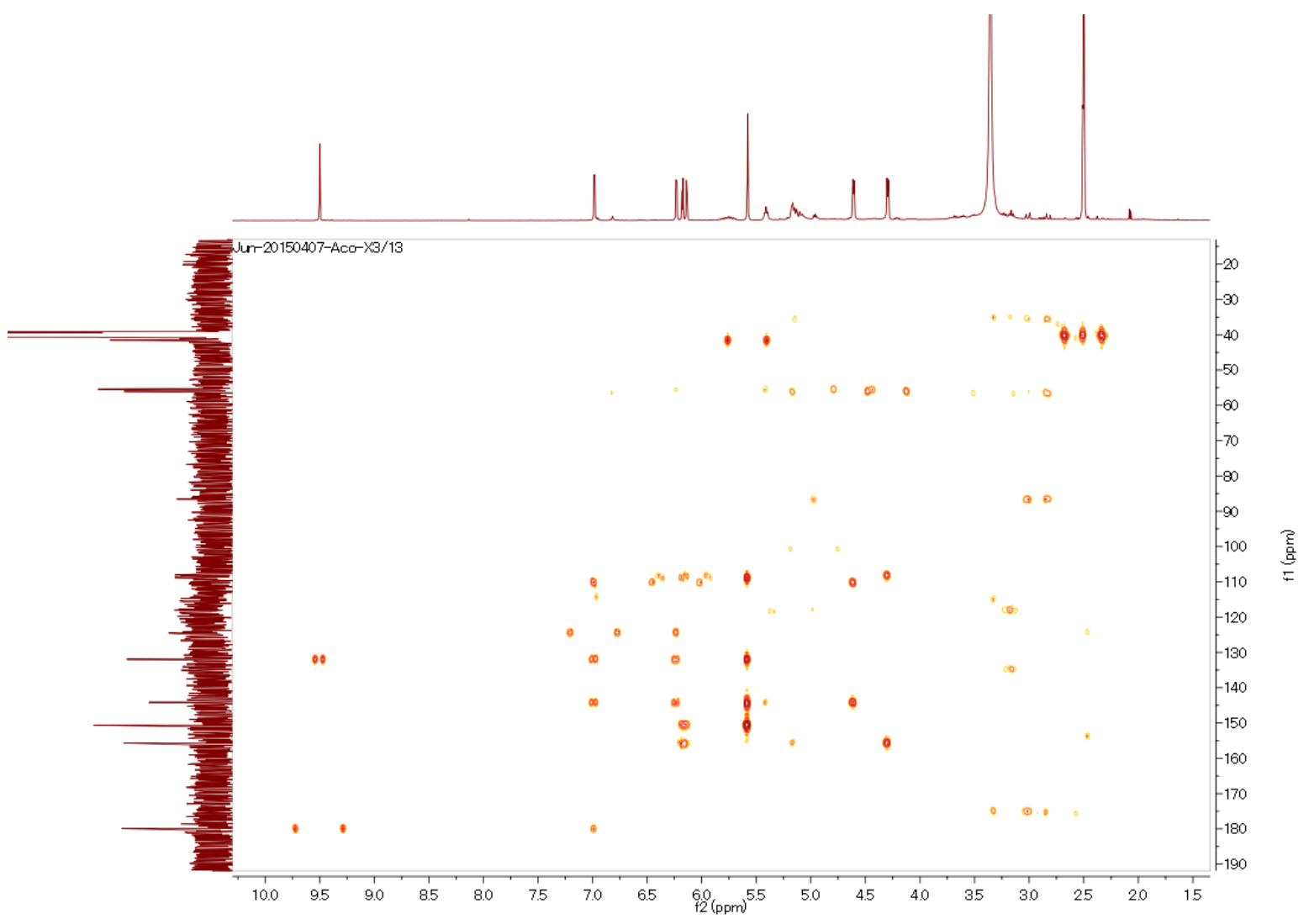

Figure S35. HMBC correlations 


\section{3-(allylthio)-2-(2-formyl-5-hydroxymethyl-1 H-pyrrol-1-yl)propanoic acid (6)}

Jun

03122013 MRFS-2Aneg 426 (1.726) Cm (423:426)

100

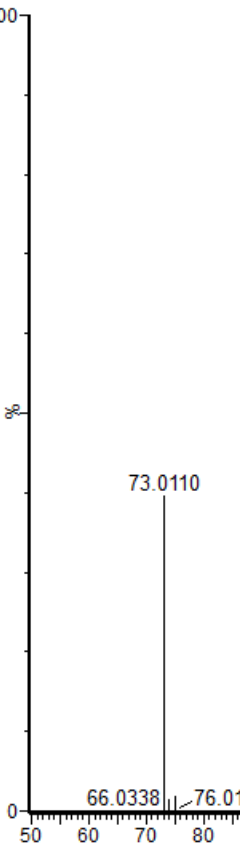

\begin{tabular}{ll|l}
124.0395 & 132.9047 & 151.0584 \\
\hline
\end{tabular}
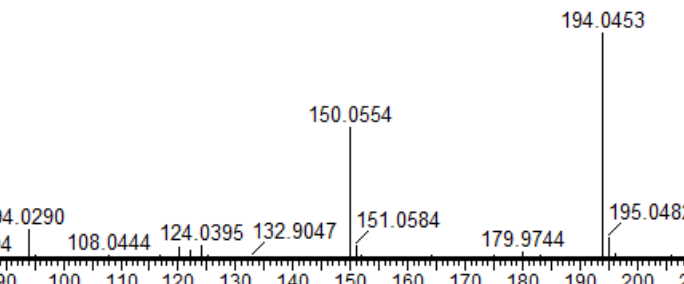

268.0642

1: TOF MS ES-

Figure S36. HRESIMS (positive) spectrum

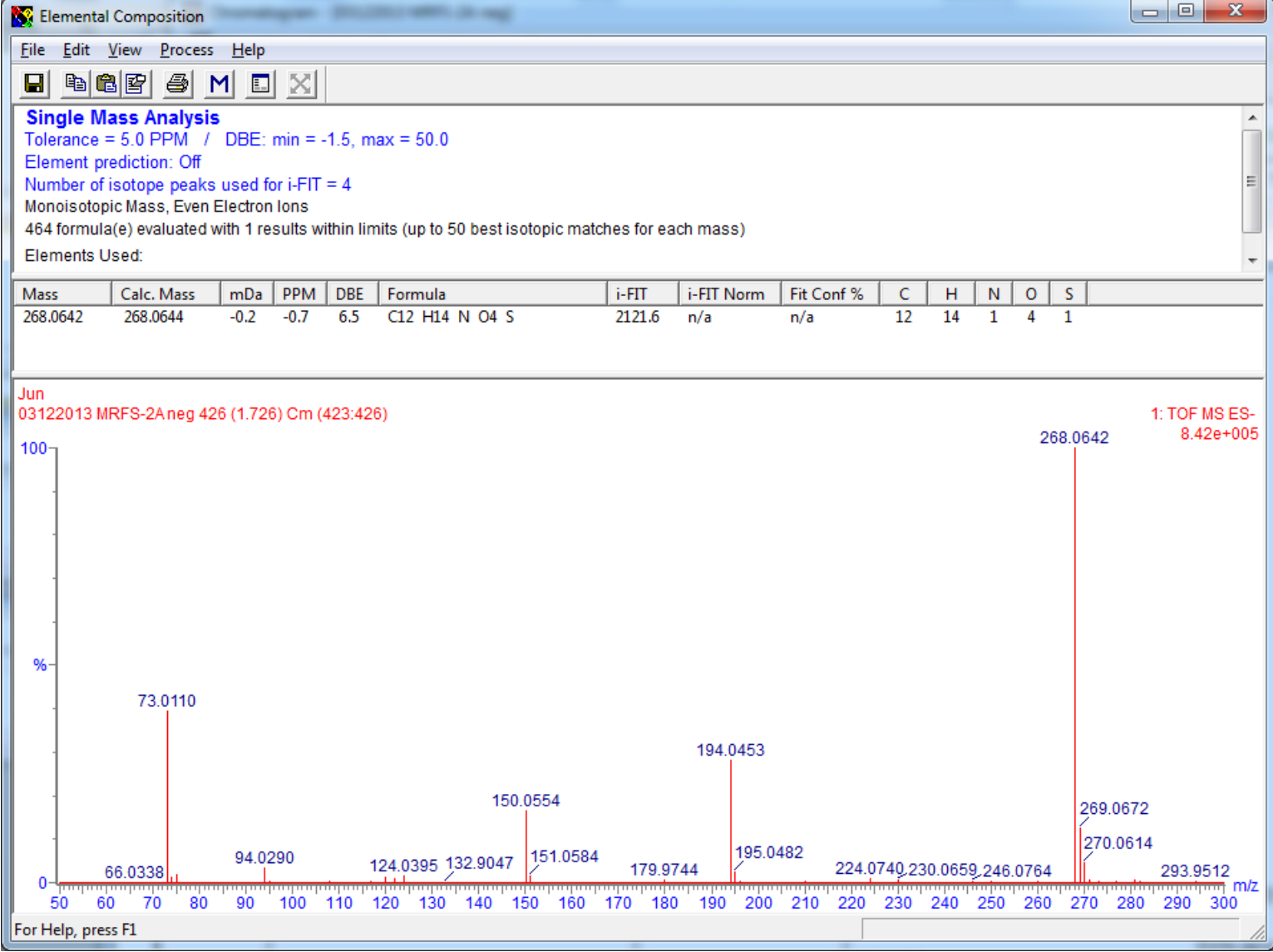

Figure S37. Elemental composition report of the precursor ion 


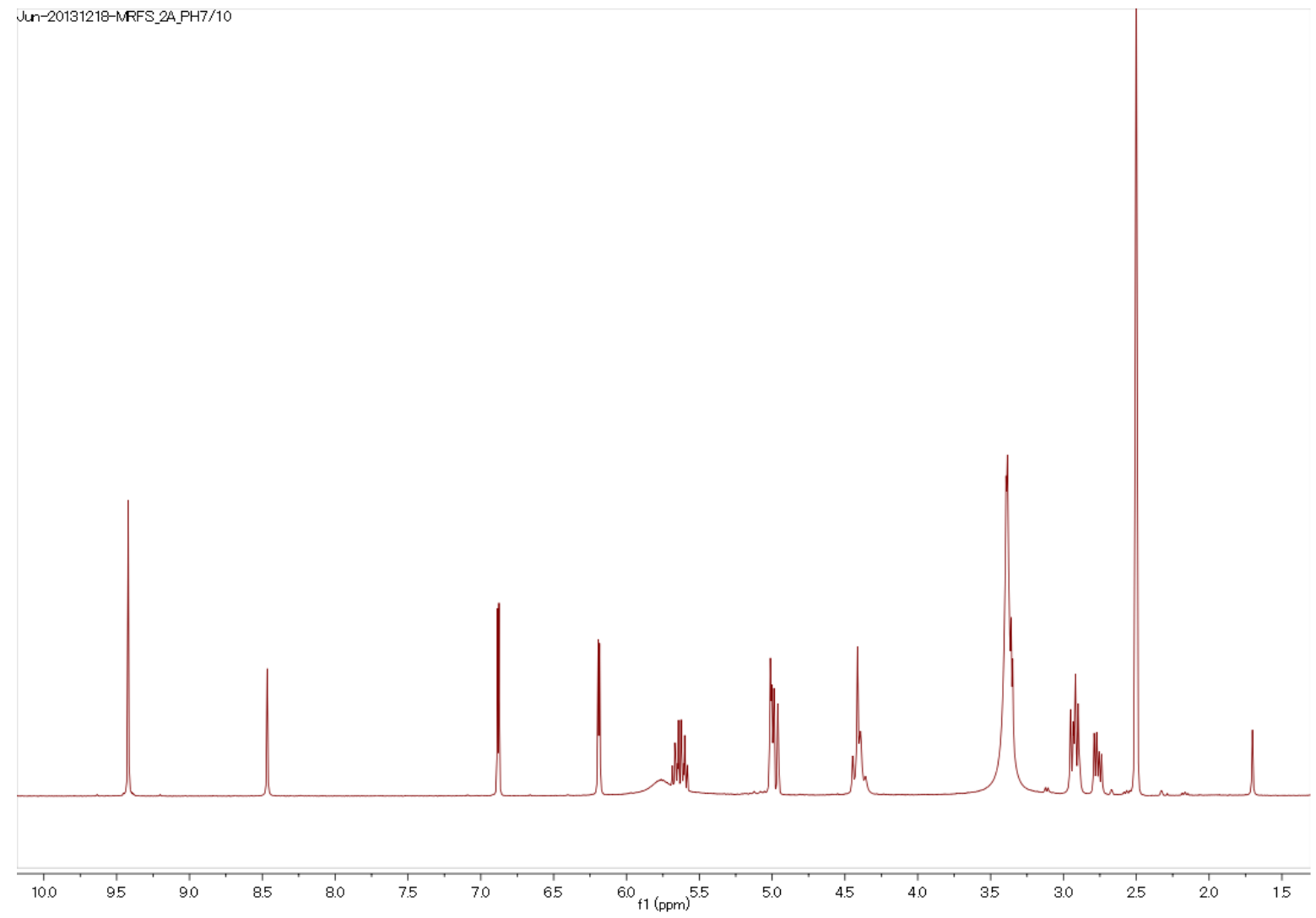

Figure S38. ${ }^{1} \mathrm{H}$ NMR spectrum (DMSO- $d_{6}, 400 \mathrm{MHz}$ )
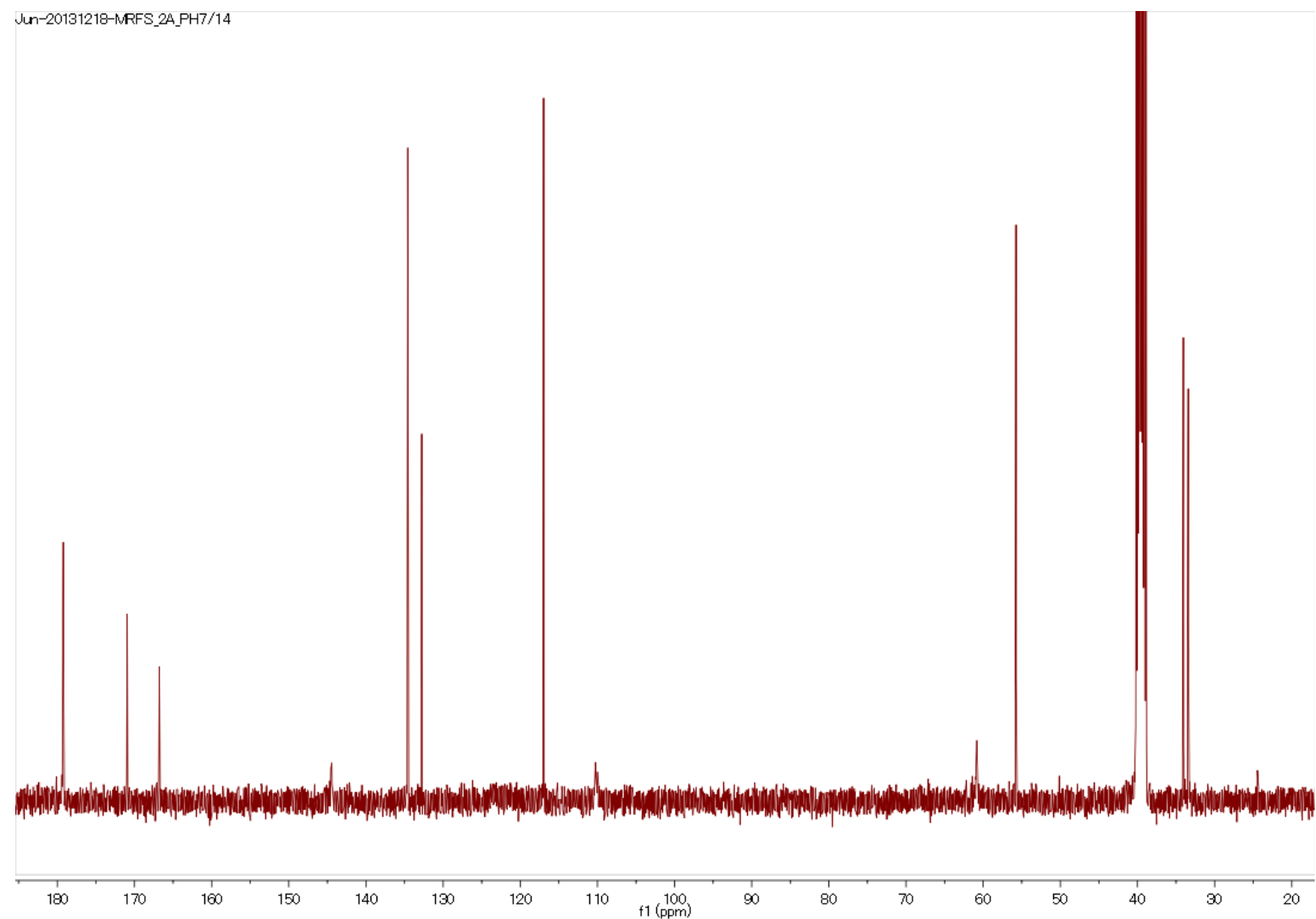

Figure S39. ${ }^{13} \mathrm{C}$ NMR spectrum (DMSO- $d_{6}, 100 \mathrm{MHz}$ ) 


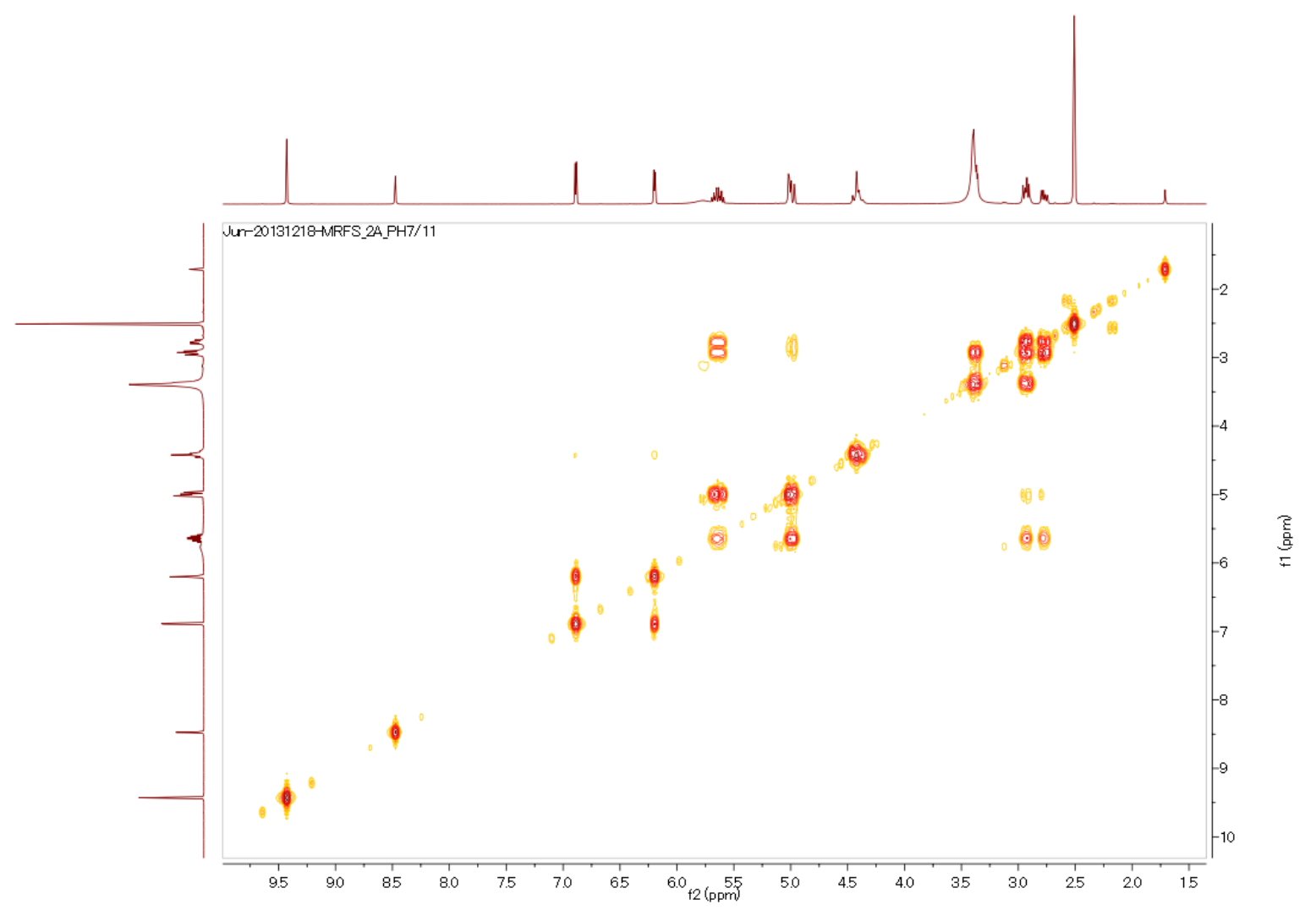

Figure S40. COSY correlations

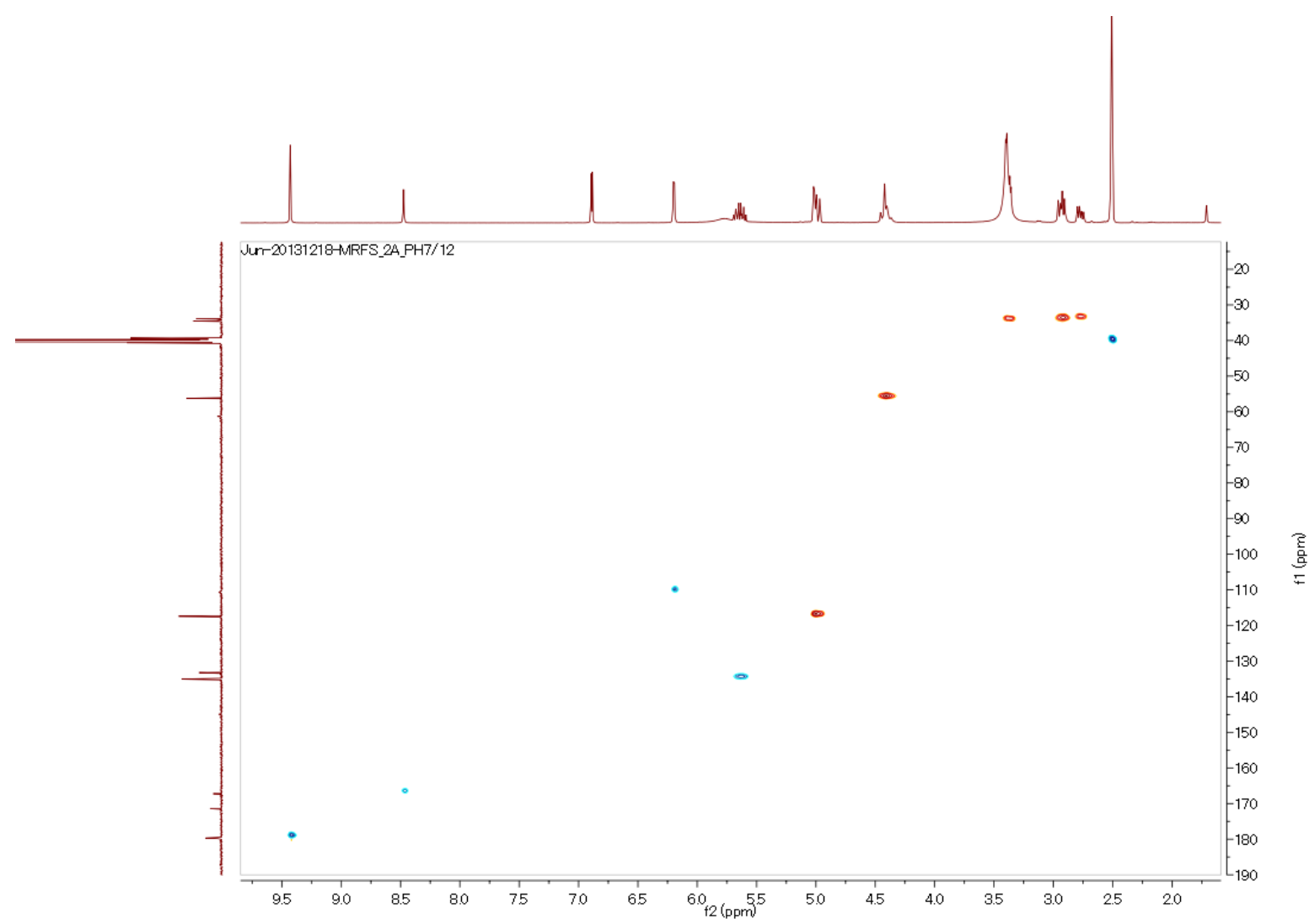

Figure S41. HSQC correlations 


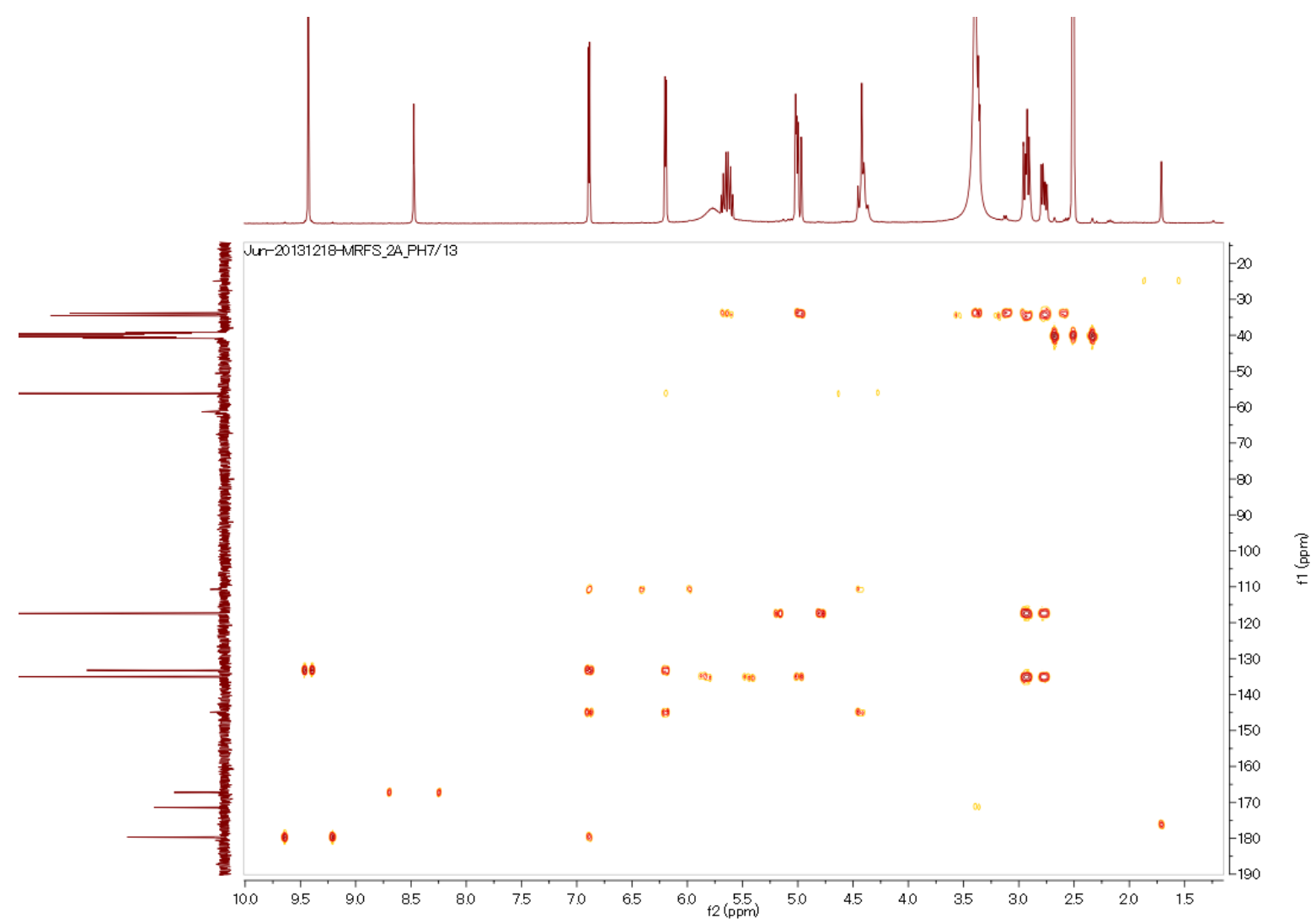

Figure S42. HMBC correlations

(4S)-4-allylthiomethyl-3,4-dihydro-3-oxo-1H-pyrrolo[2,1-c][1,4]oxazine-6carbaldehyde (7)

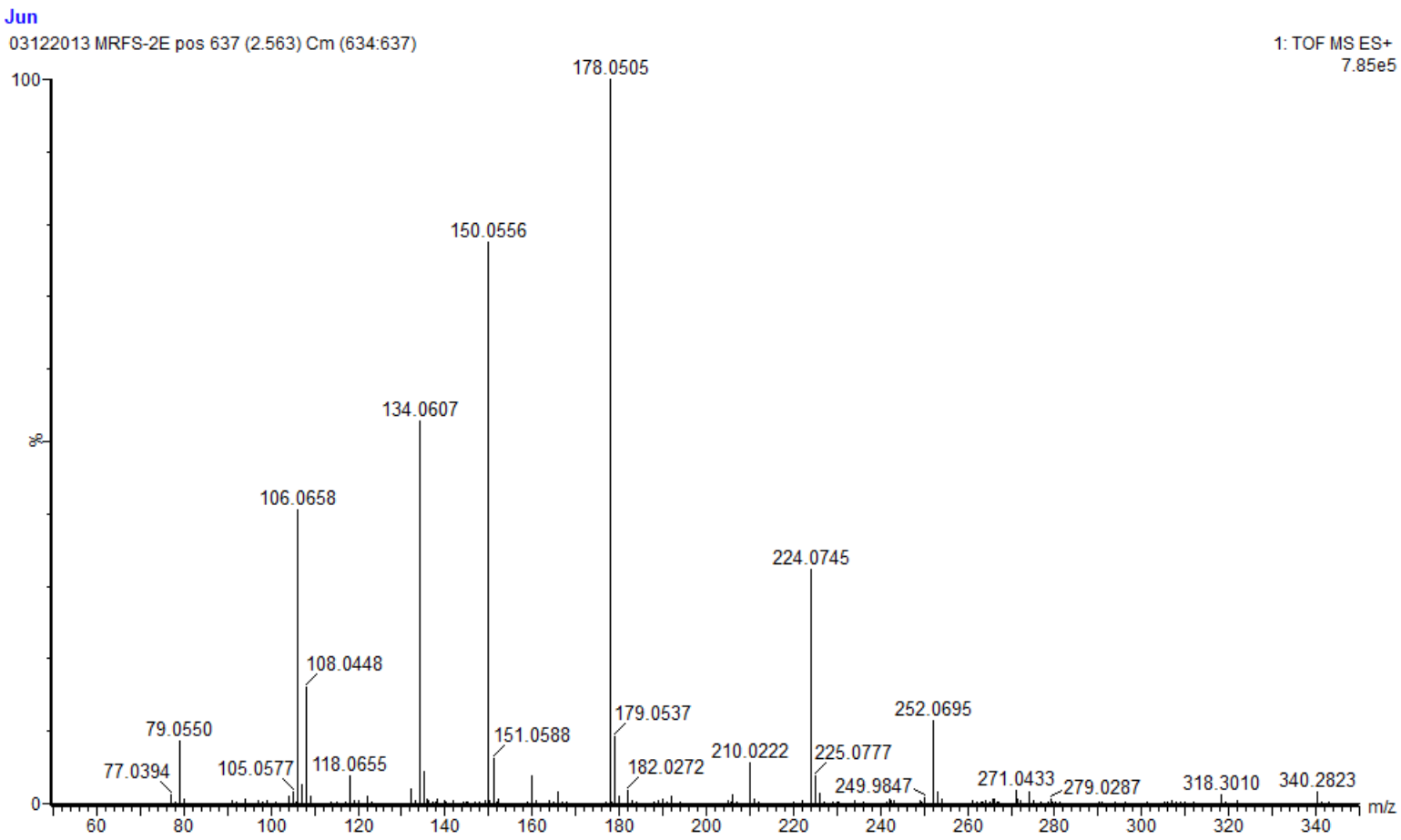

Figure S43. HRESIMS (positive) spectrum 


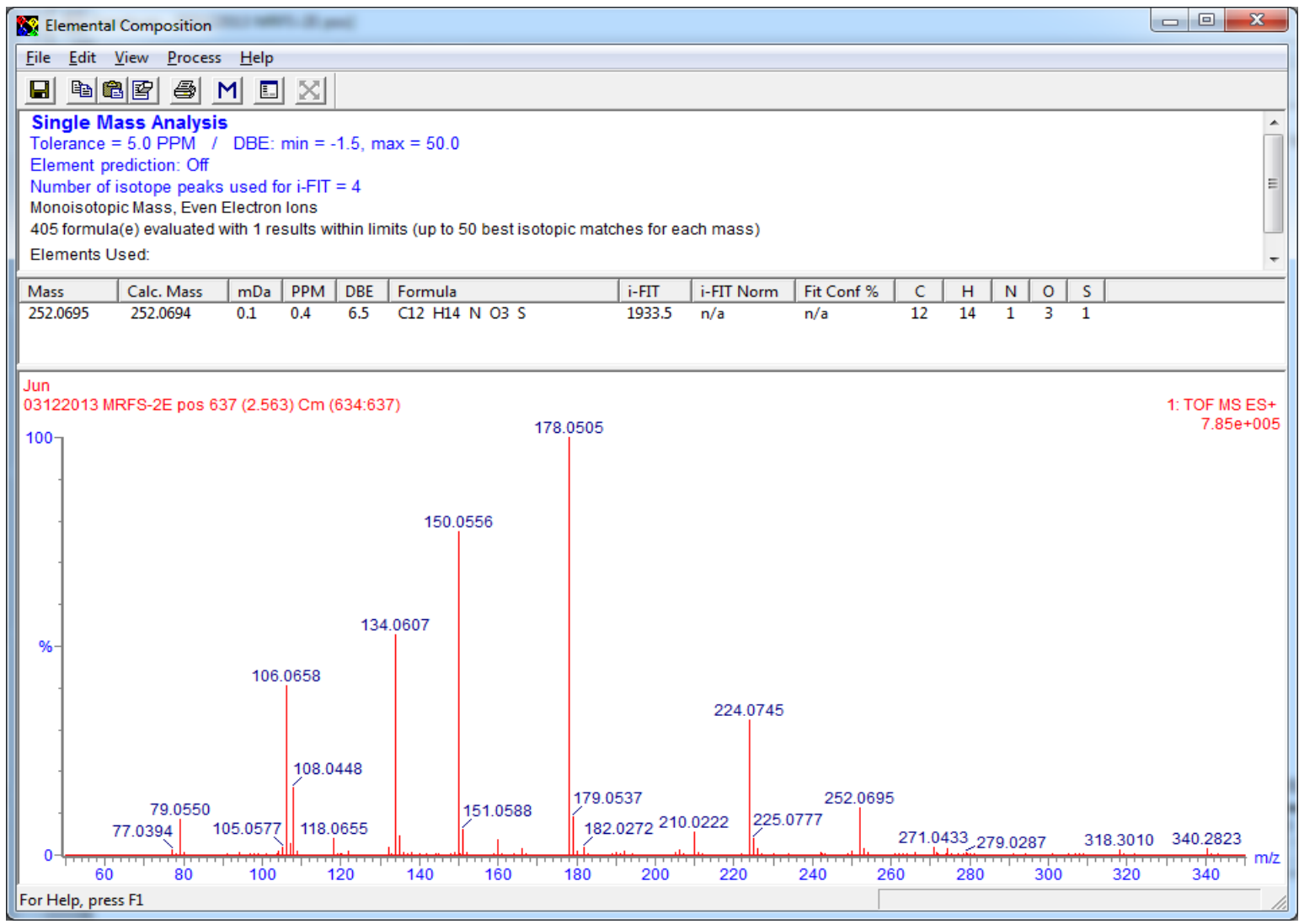

Figure S44. Elemental composition report of the precursor ion

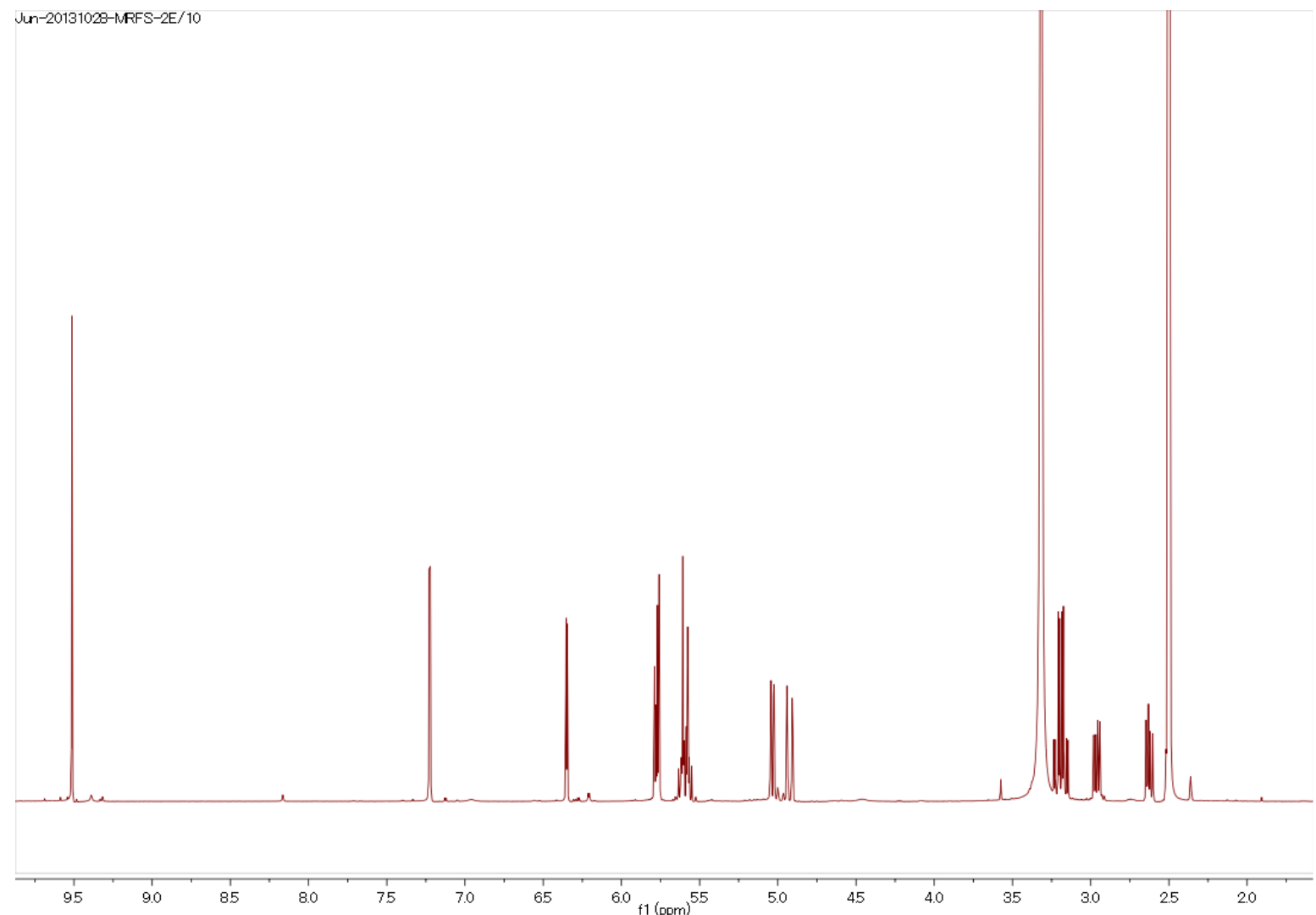

Figure S45. ${ }^{1} \mathrm{H}$ NMR spectrum (DMSO- $d_{6}, 500 \mathrm{MHz}$ ) 

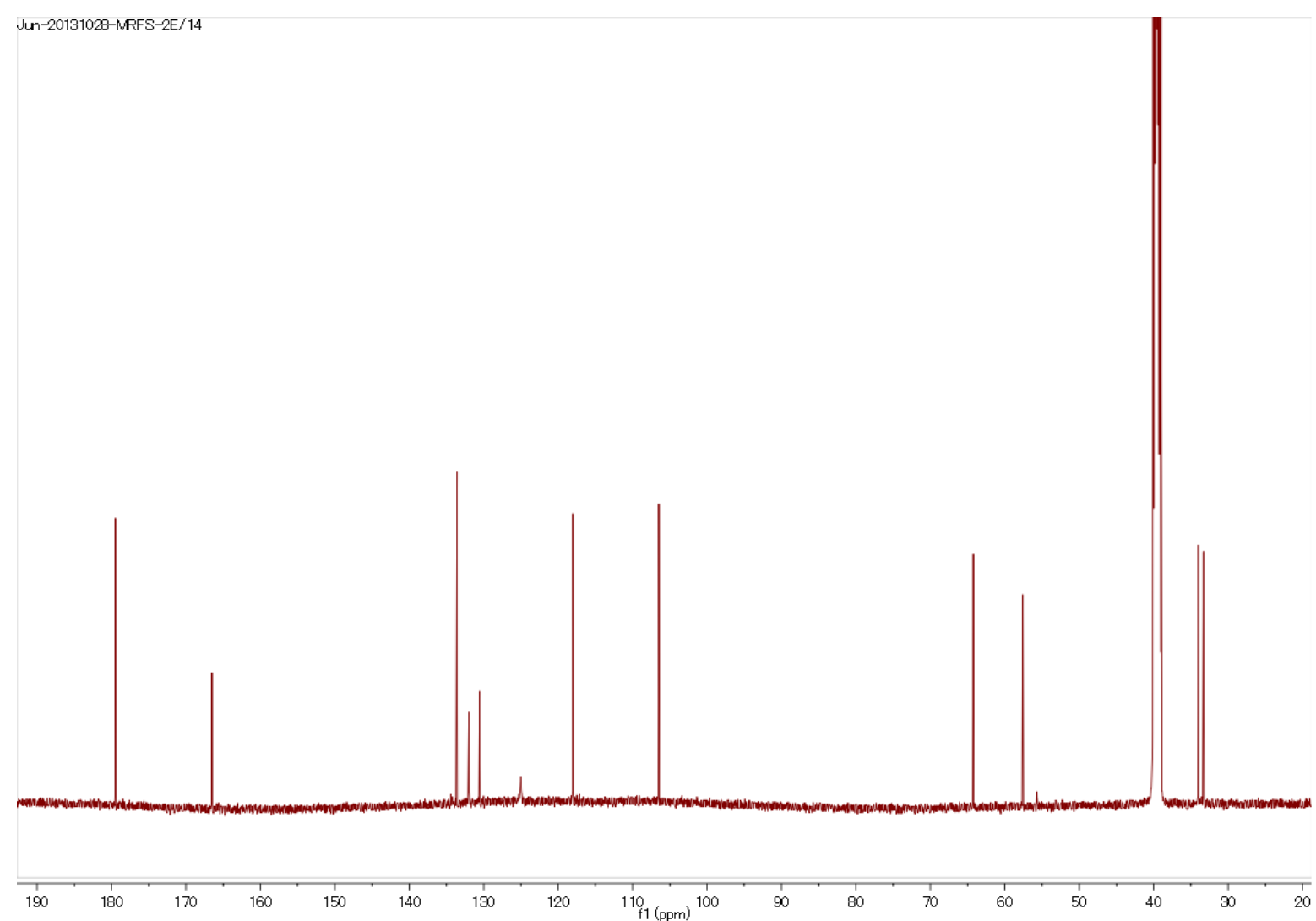

Figure S46. ${ }^{13} \mathrm{C}$ NMR spectrum (DMSO- $d_{6}, 125 \mathrm{MHz}$ )

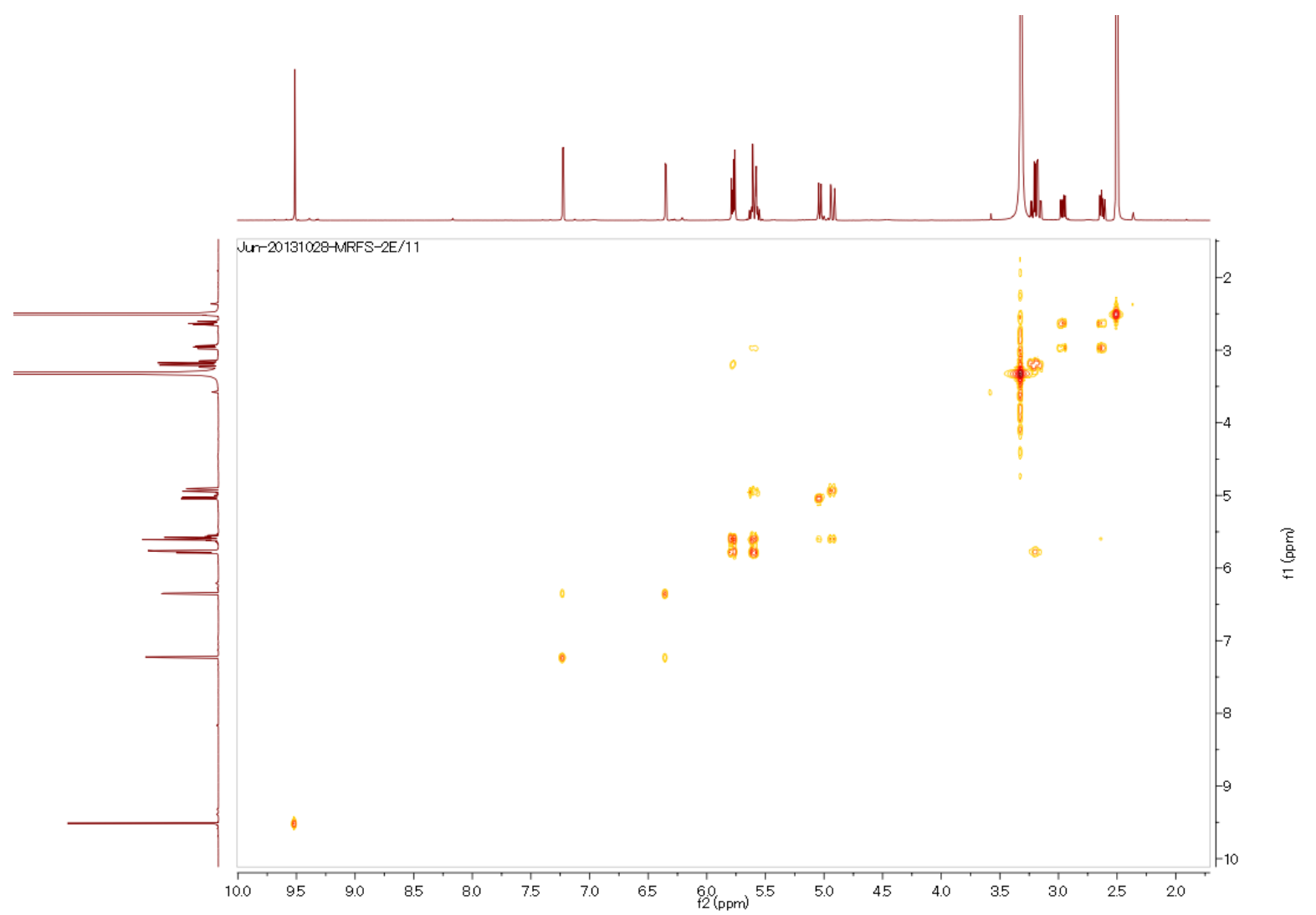

Figure S47. COSY correlations 


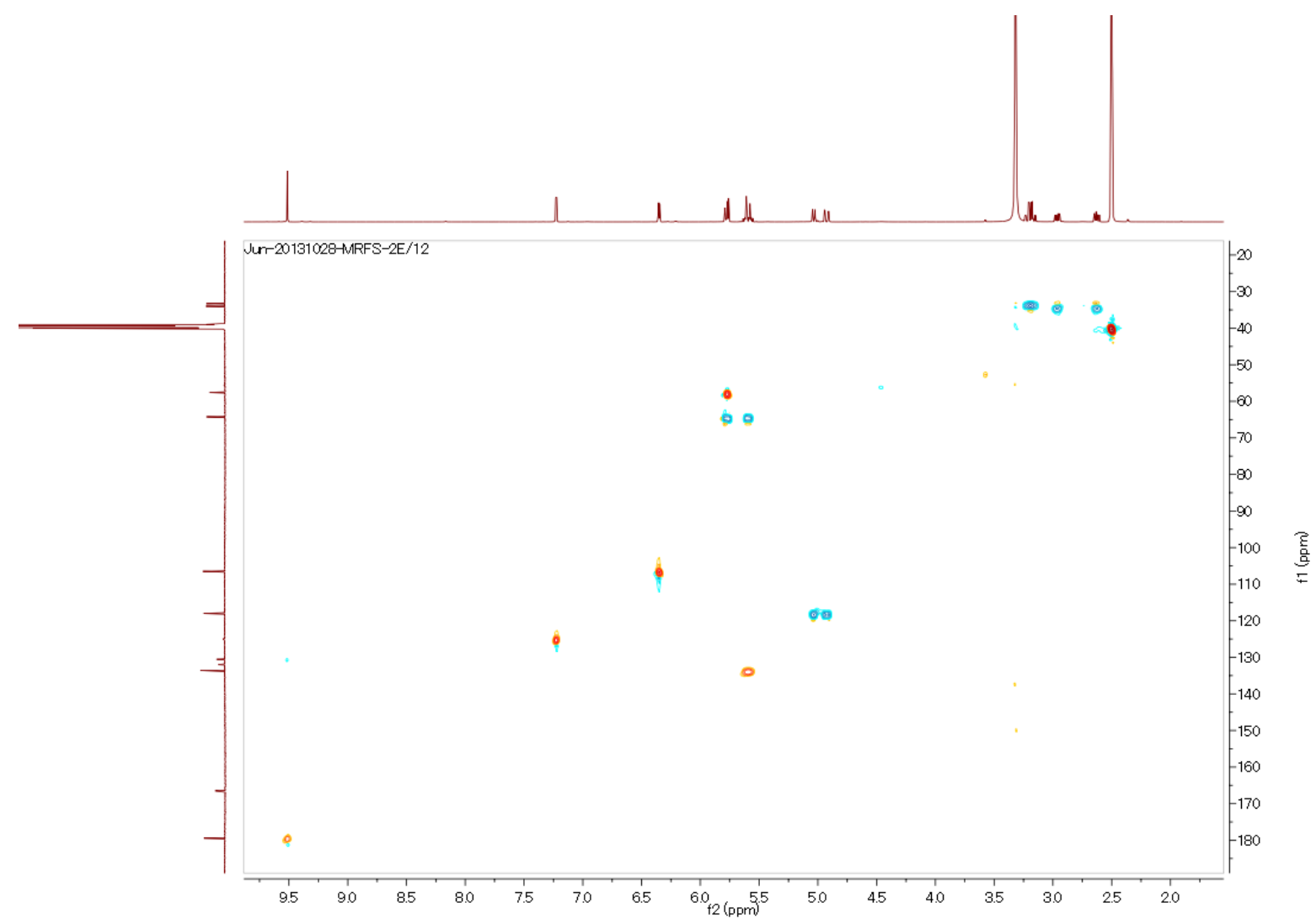

Figure S48. HSQC correlations

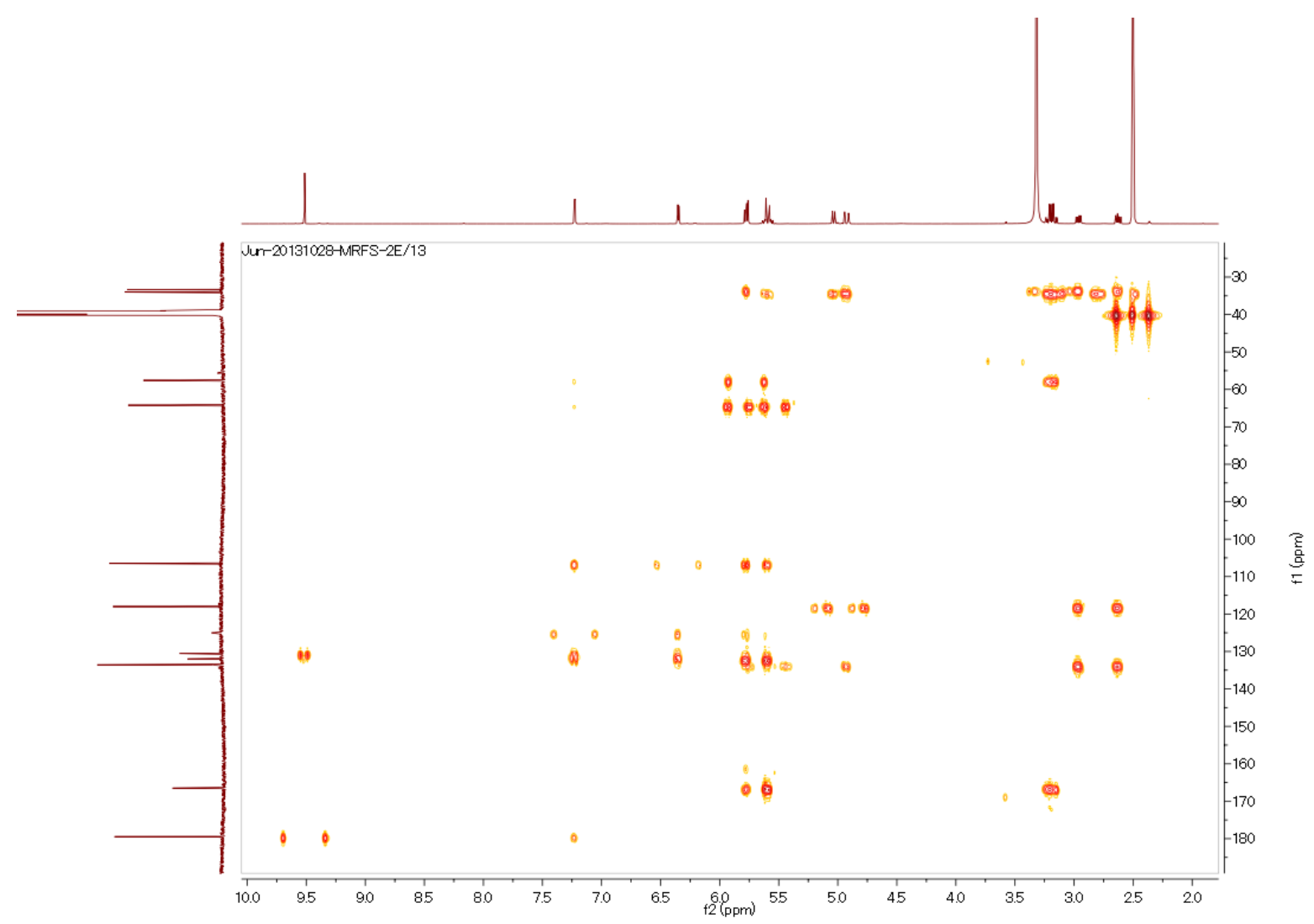

Figure S49. HMBC correlations 
(2R)-3-(allylthio)-2-((4R)-4-(allylthiomethyl)-6-formyl-3-oxo-3,4-dihydropyr rolo[1,2-a]- pyrazin-2(1H)-yl)propanoic acid (8)

Jun

03122013 MRFS-5 neg
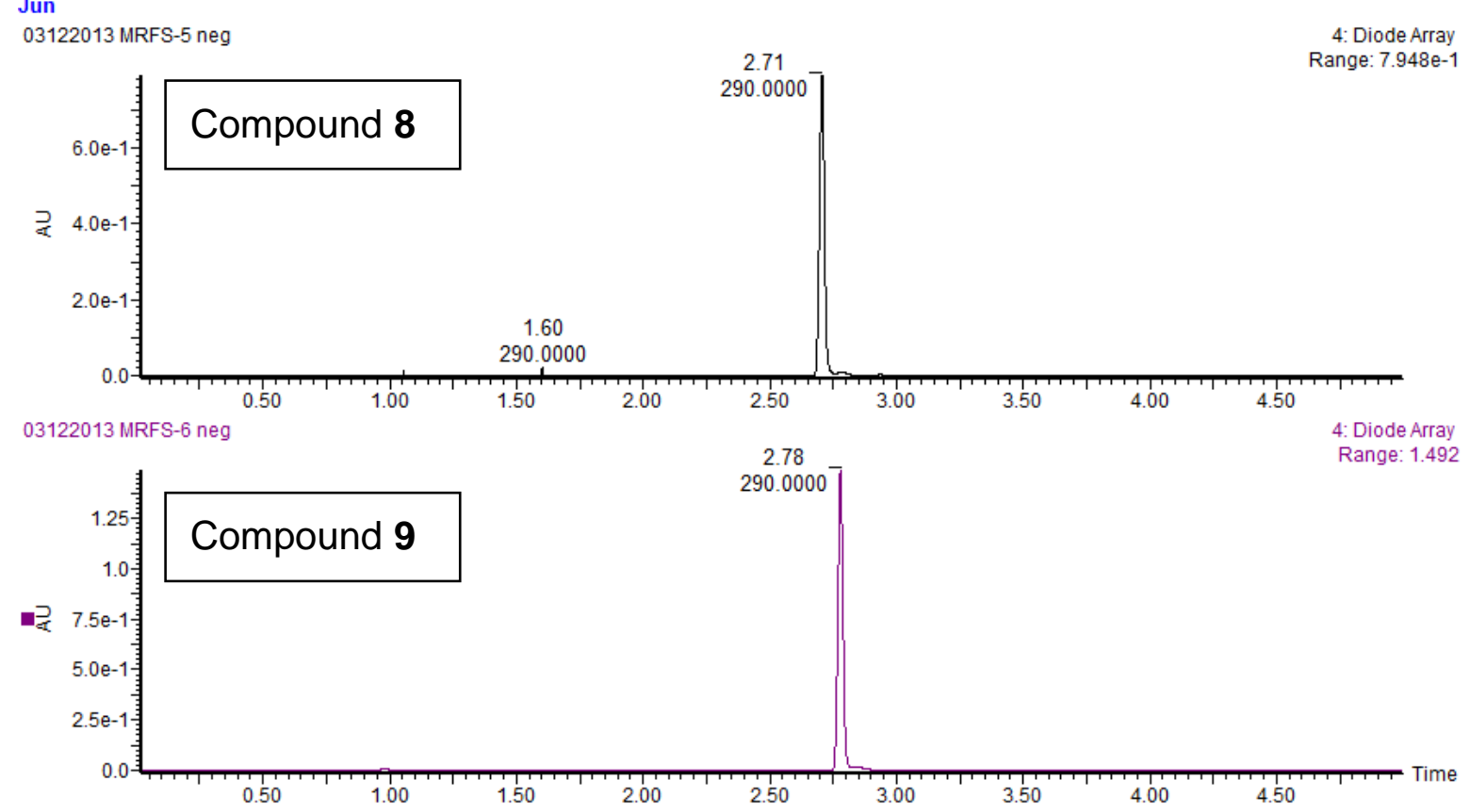

Figure S50. Chromatograms of compounds 8 and $\mathbf{9}$ recorded by UV detection (290 $\mathrm{nm}$ ) using UPLC-TOF-MS

Jun

03122013 MRFS-5 neg 684 (2.757)

${ }^{100}$

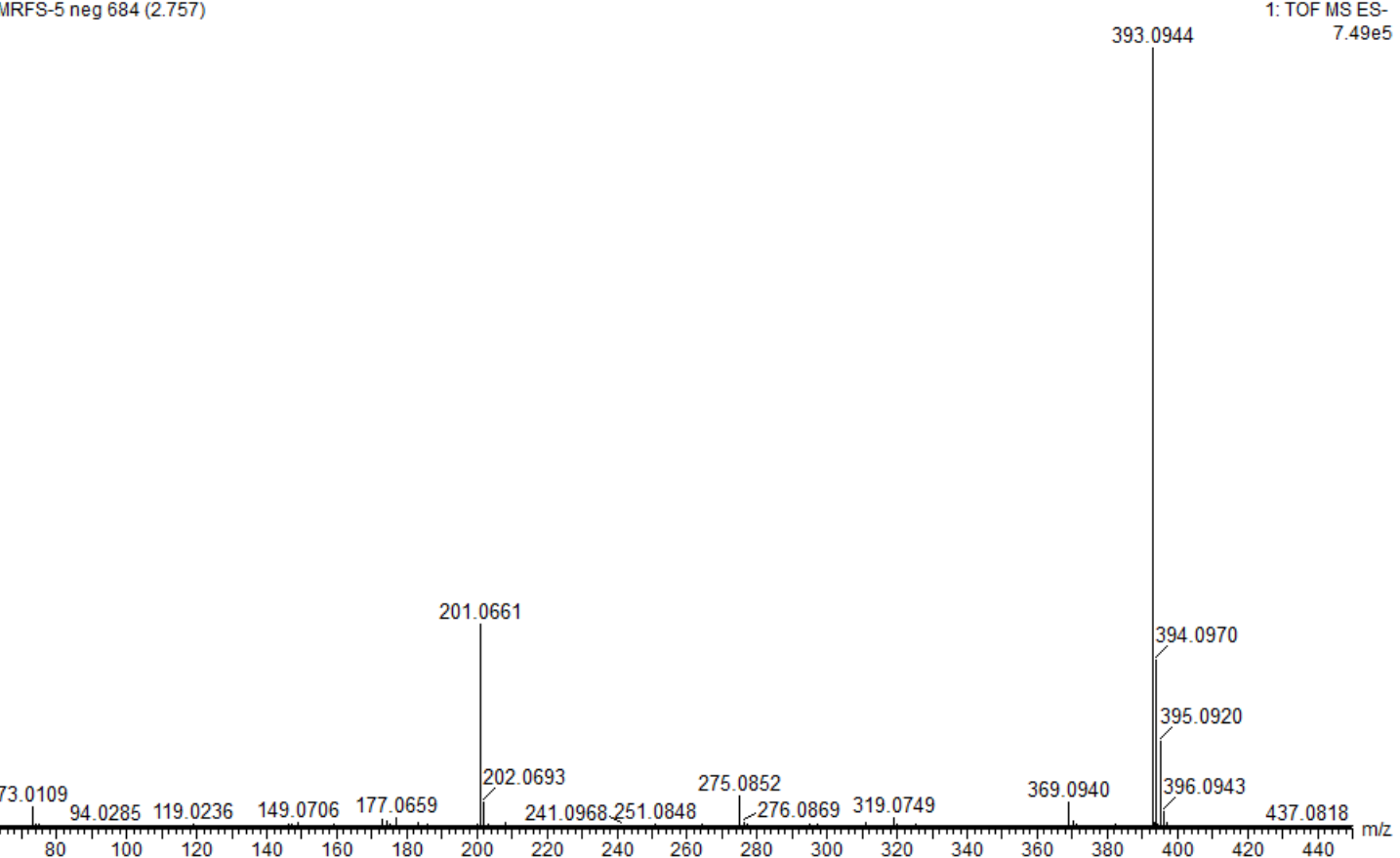

Figure S51. HRESIMS (negative) spectrum 


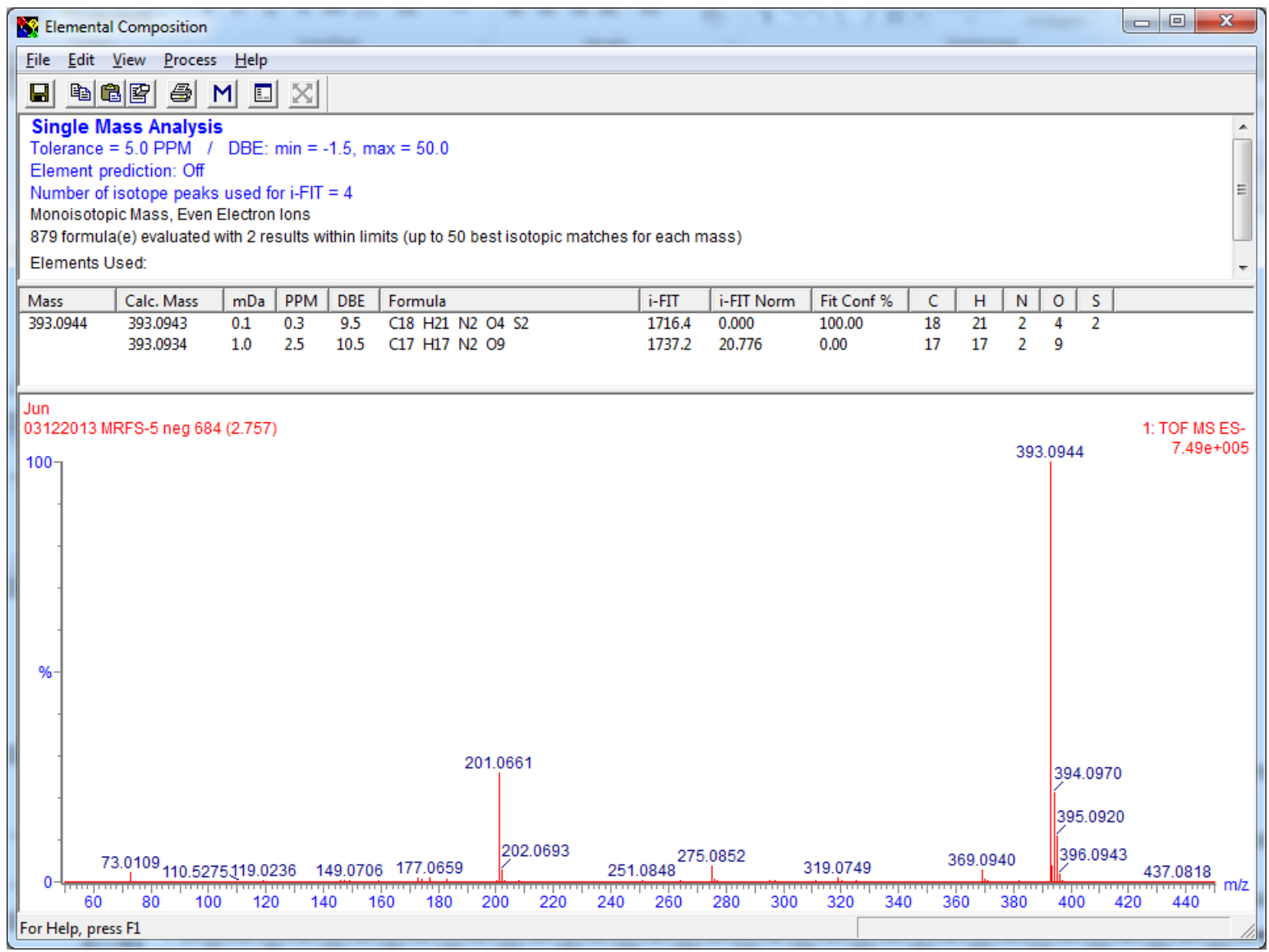

Figure S52. Elemental composition report of the precursor ion

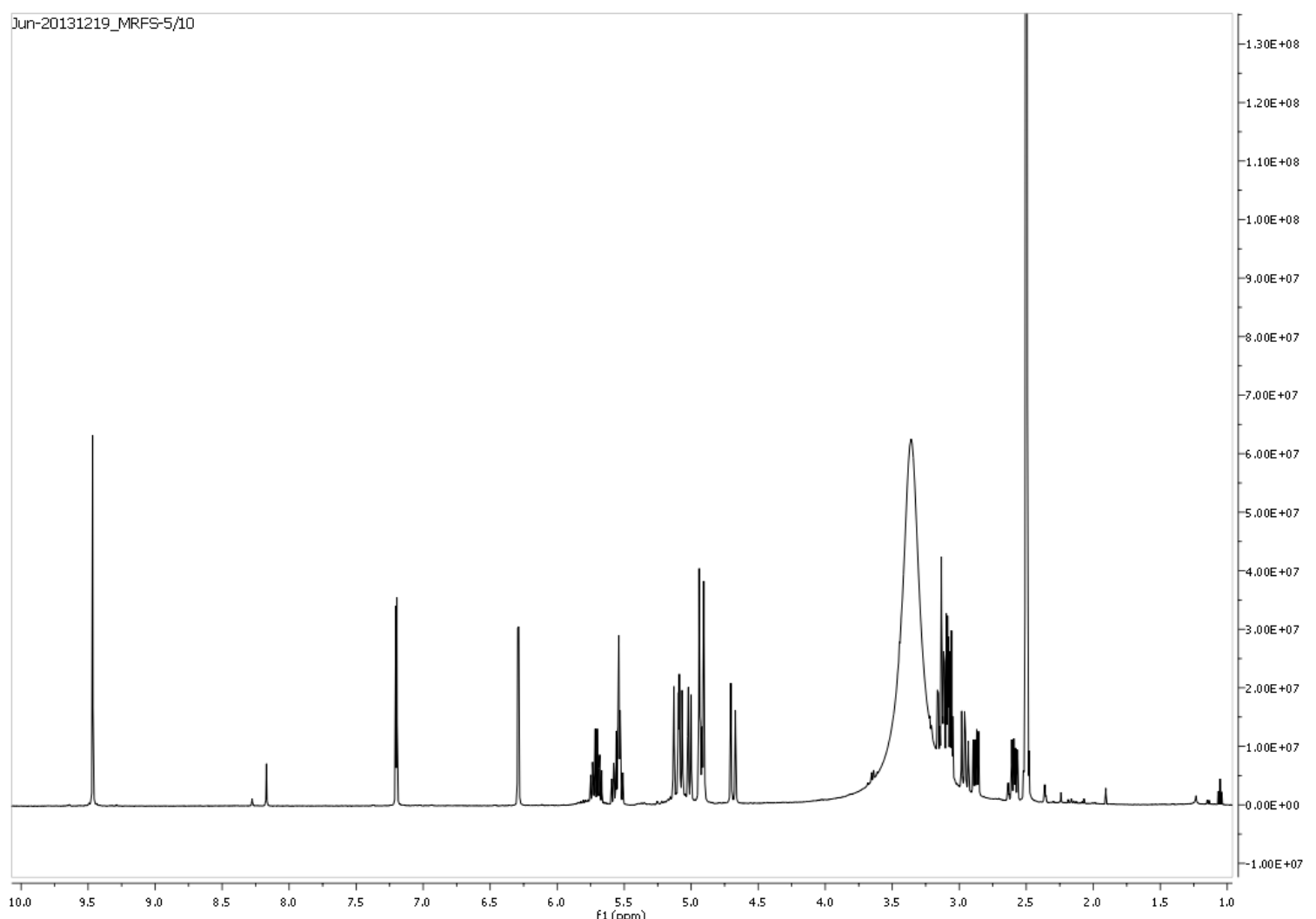

Figure S53. ${ }^{1} \mathrm{H}$ NMR spectrum (DMSO- $d_{6}, 500 \mathrm{MHz}$ ) 


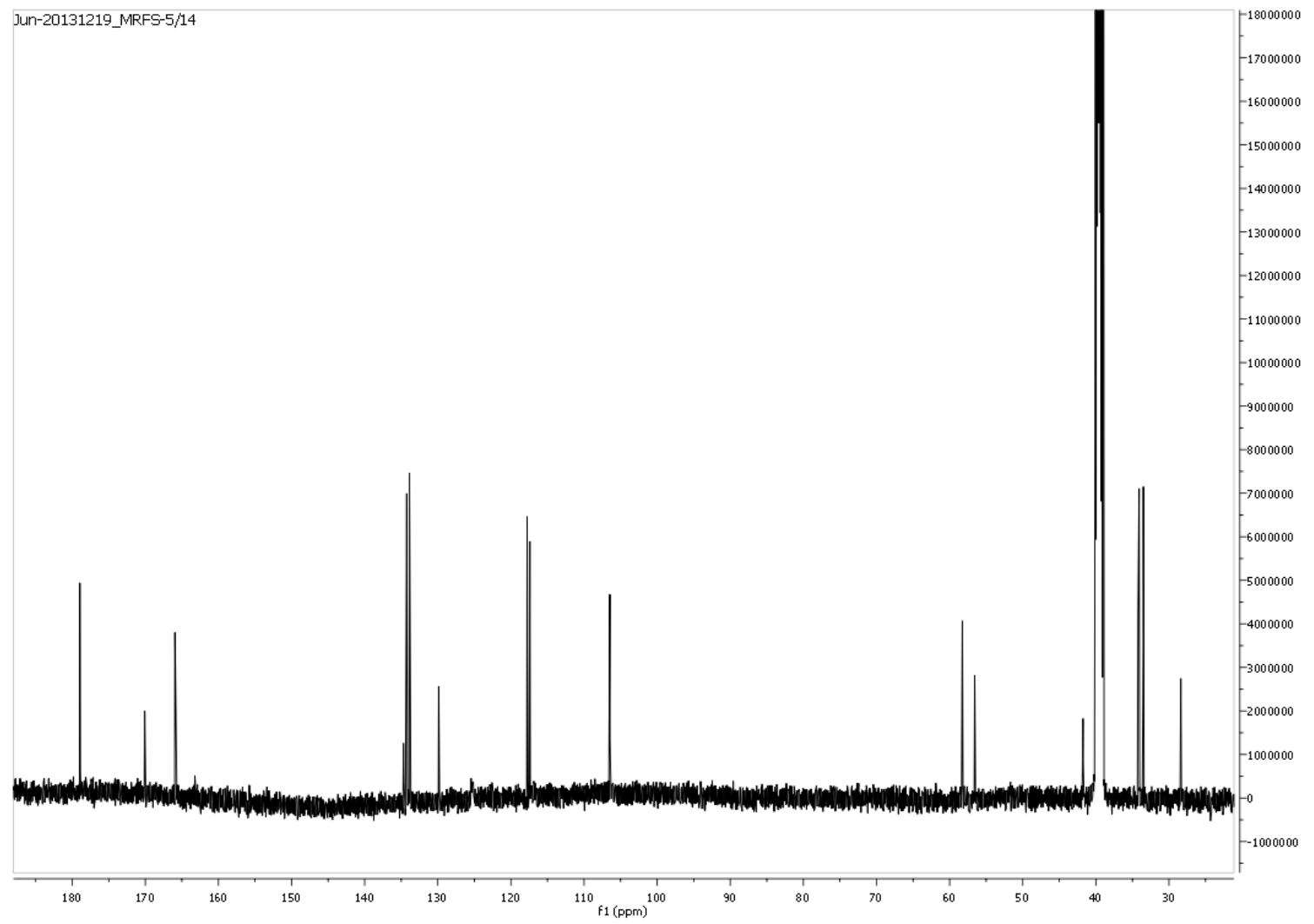

Figure S54. ${ }^{13} \mathrm{C}$ NMR spectrum (DMSO- $d_{6}, 125 \mathrm{MHz}$ )

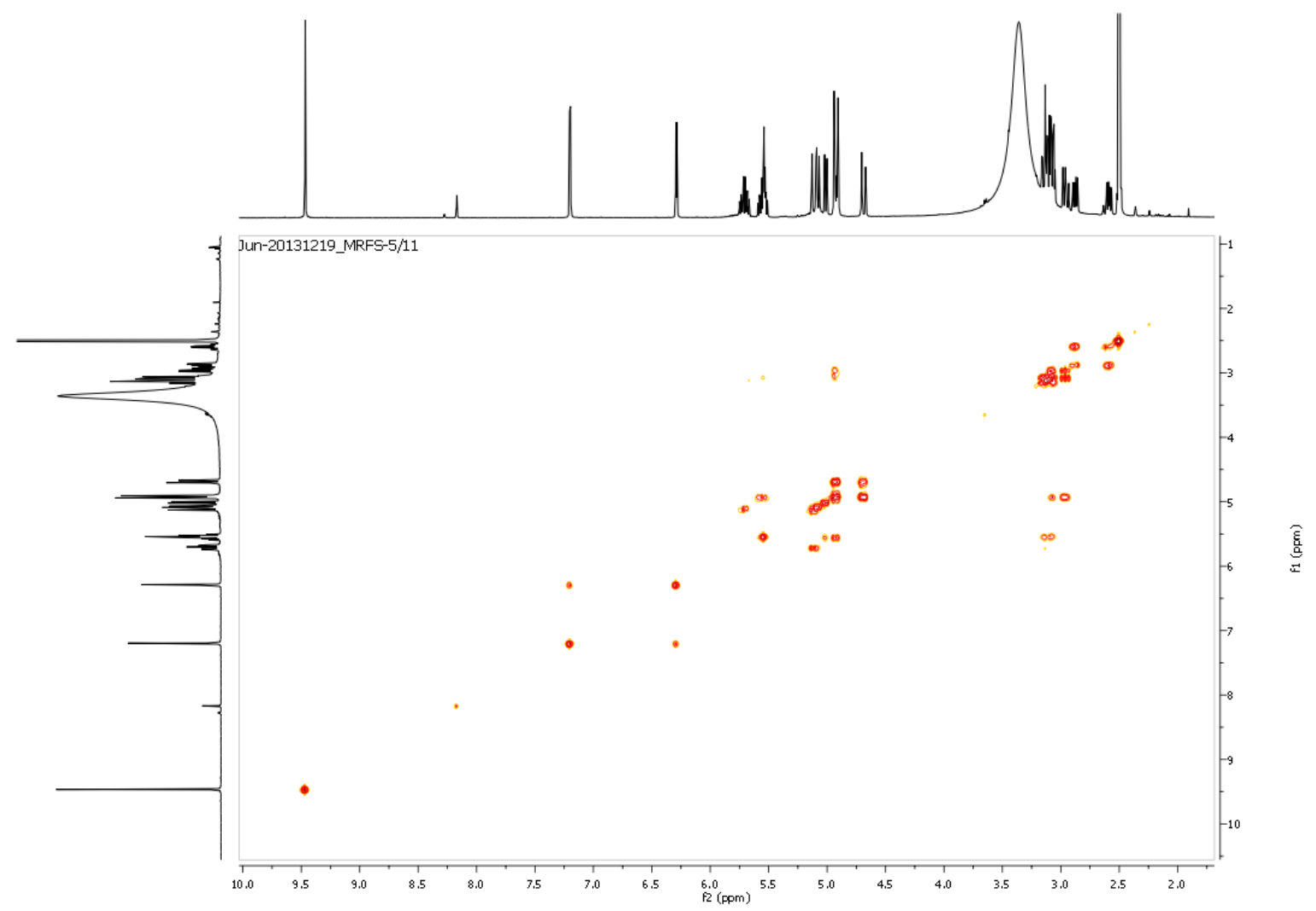

Figure S55. COSY correlations 


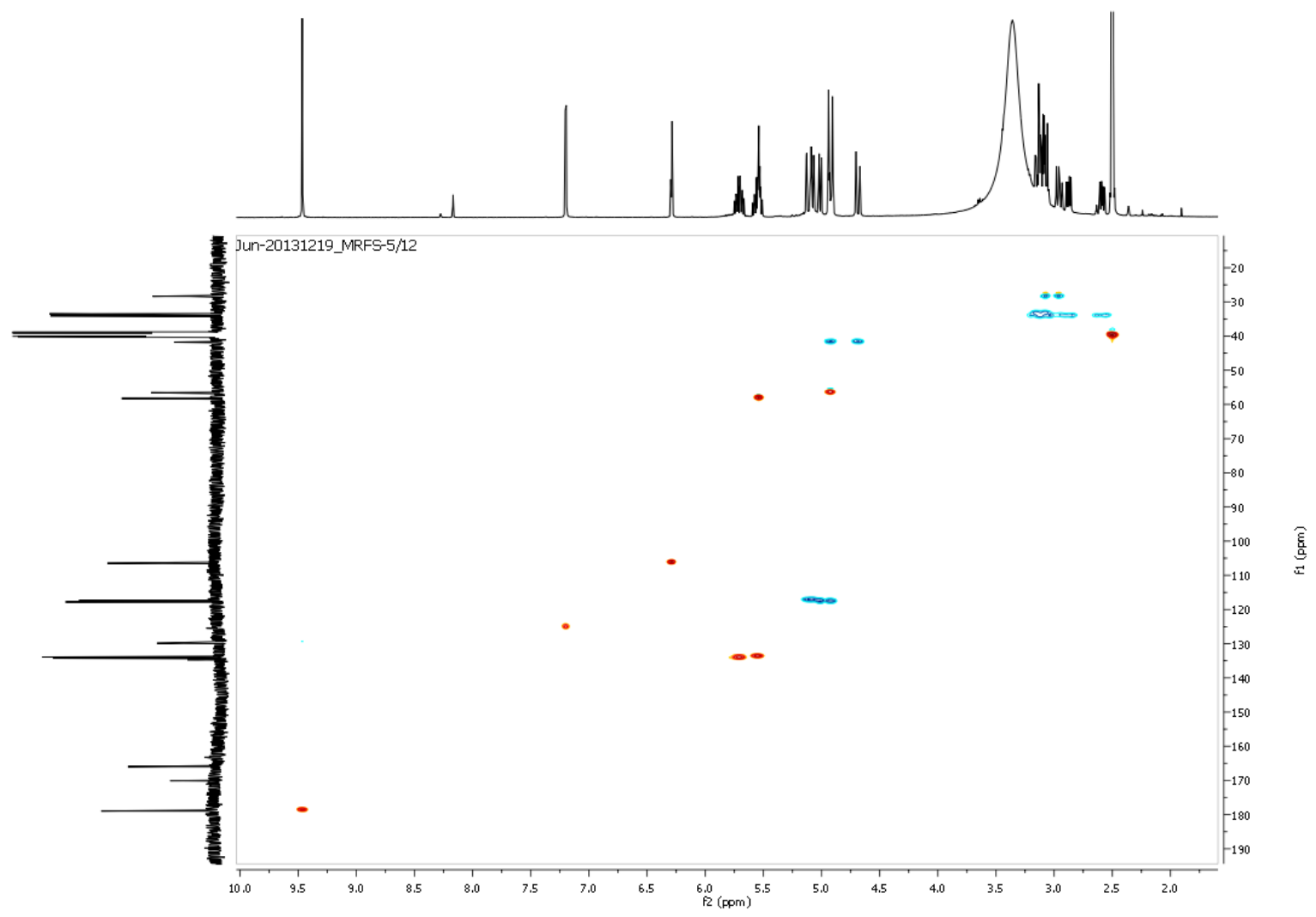

Figure S56. HSQC correlations

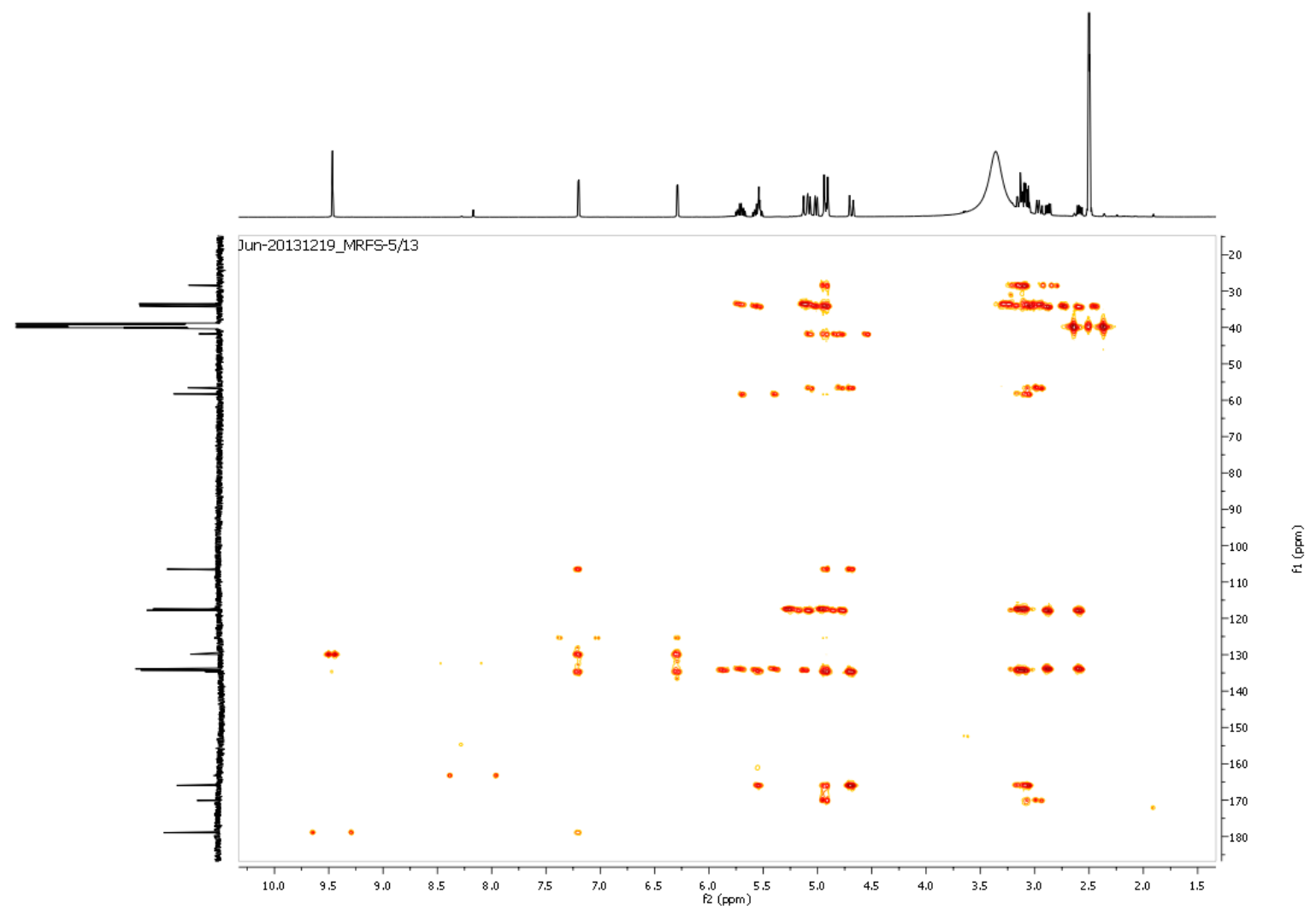

Figure S57. HMBC correlations 
(2R)-3-(allylthio)-2-((4S)-4-(allylthiomethyl)-6-formyl-3-oxo-3,4-dihydropyr rolo[1,2-a]- pyrazin-2(1H)-yl)propanoic acid (9)

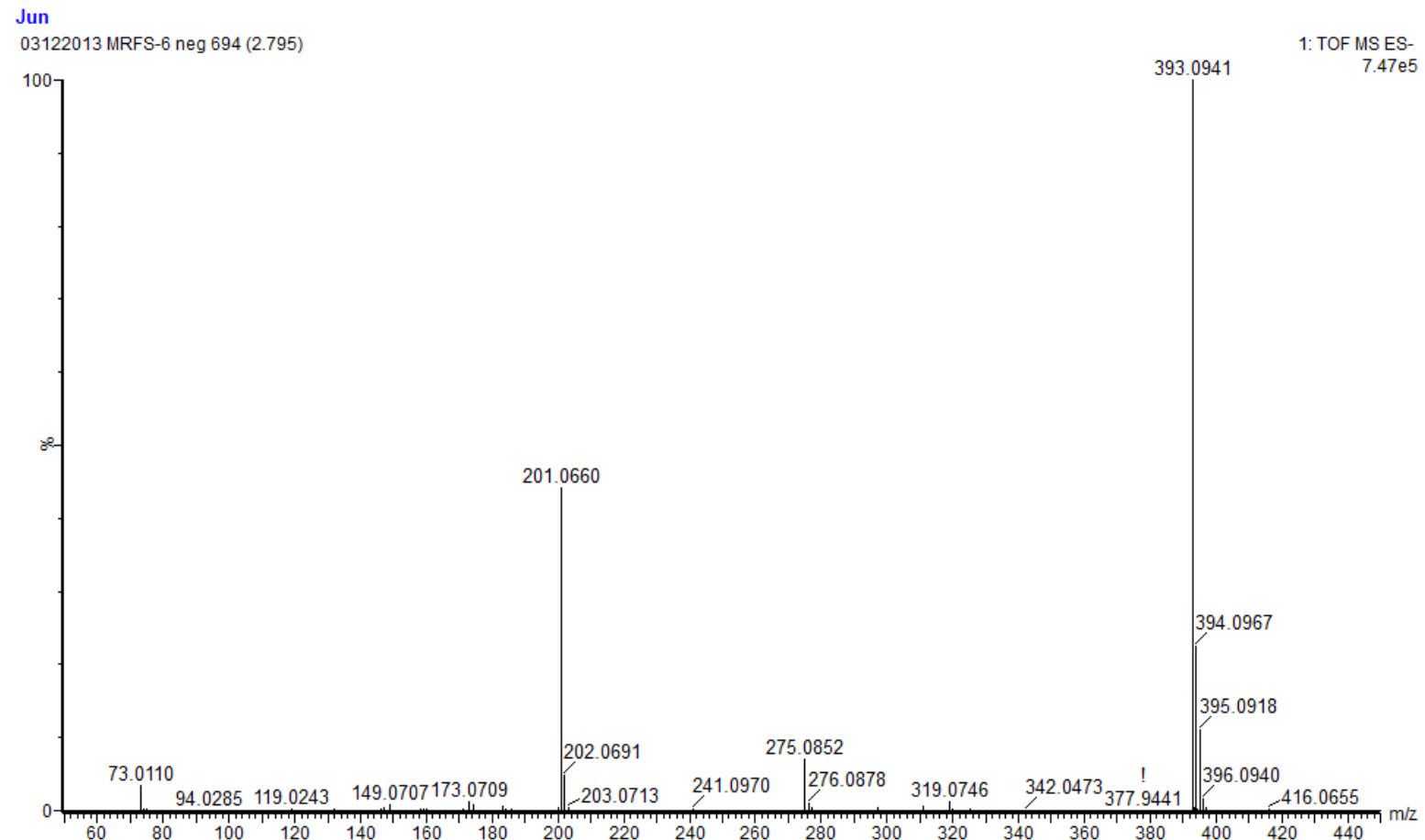

Figure S58. HRESIMS (negative) spectrum

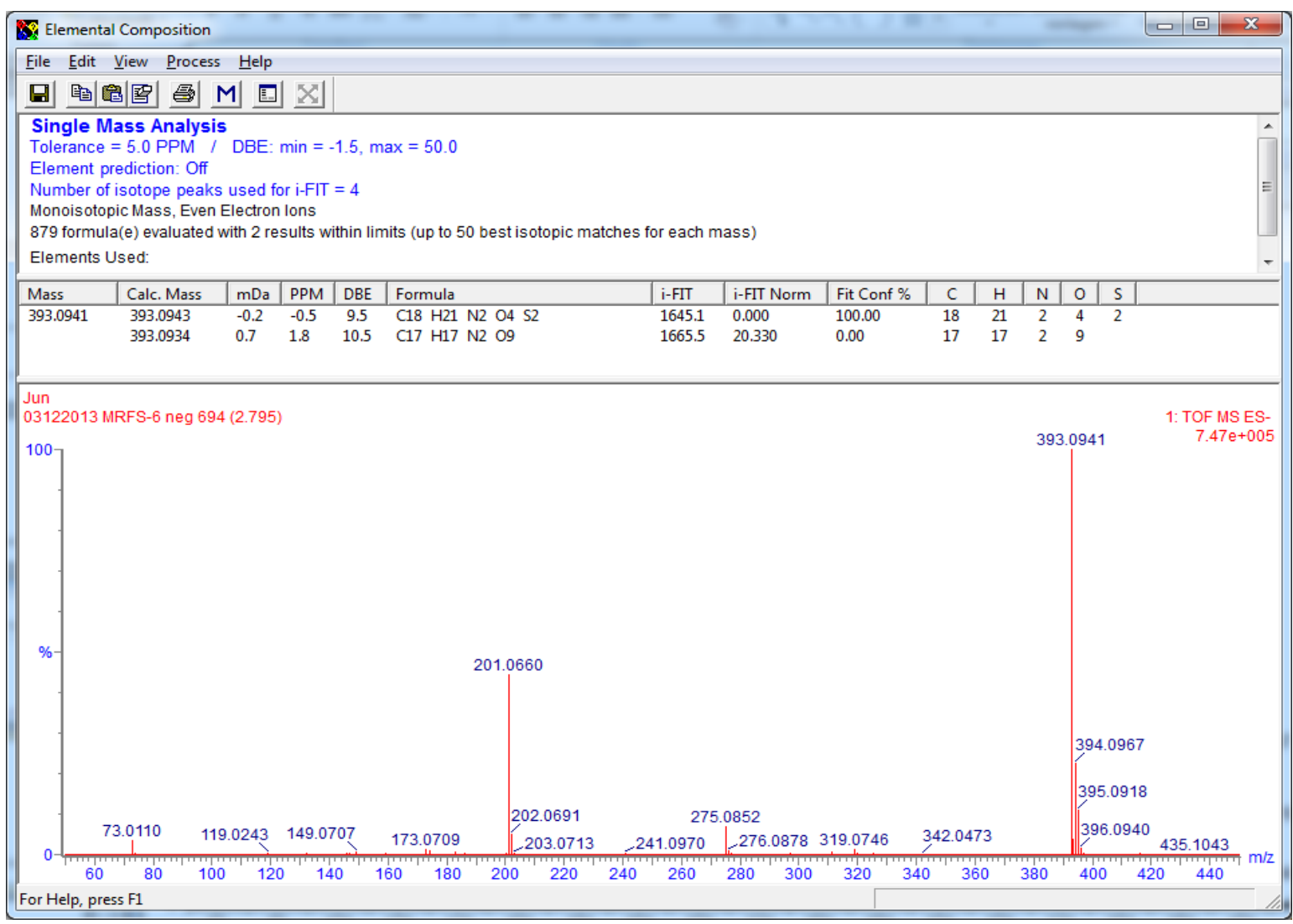

Figure S59. Elemental composition report of the precursor ion 


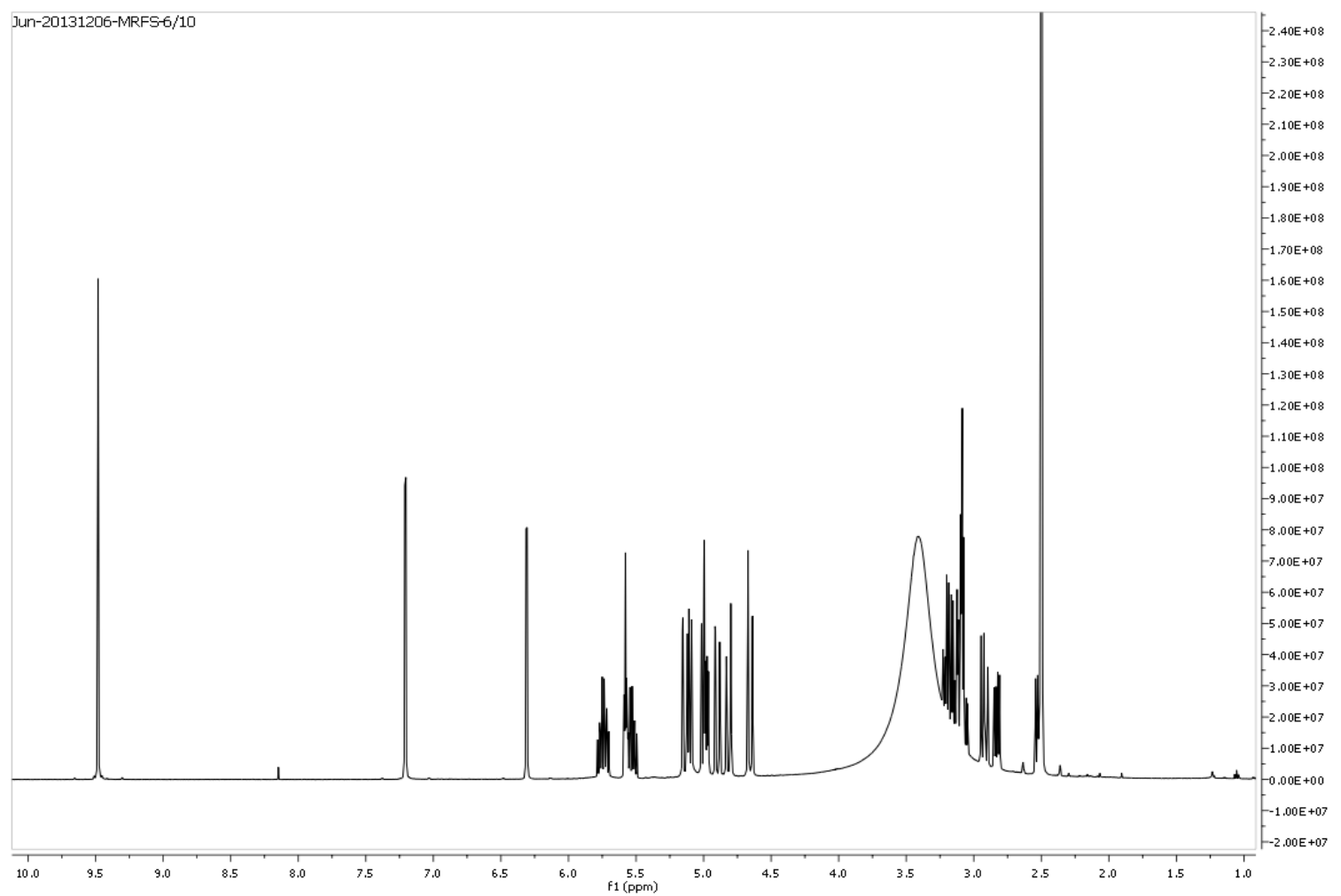

Figure S60. ${ }^{1} \mathrm{H}$ NMR spectrum (DMSO- $d_{6}, 500 \mathrm{MHz}$ )

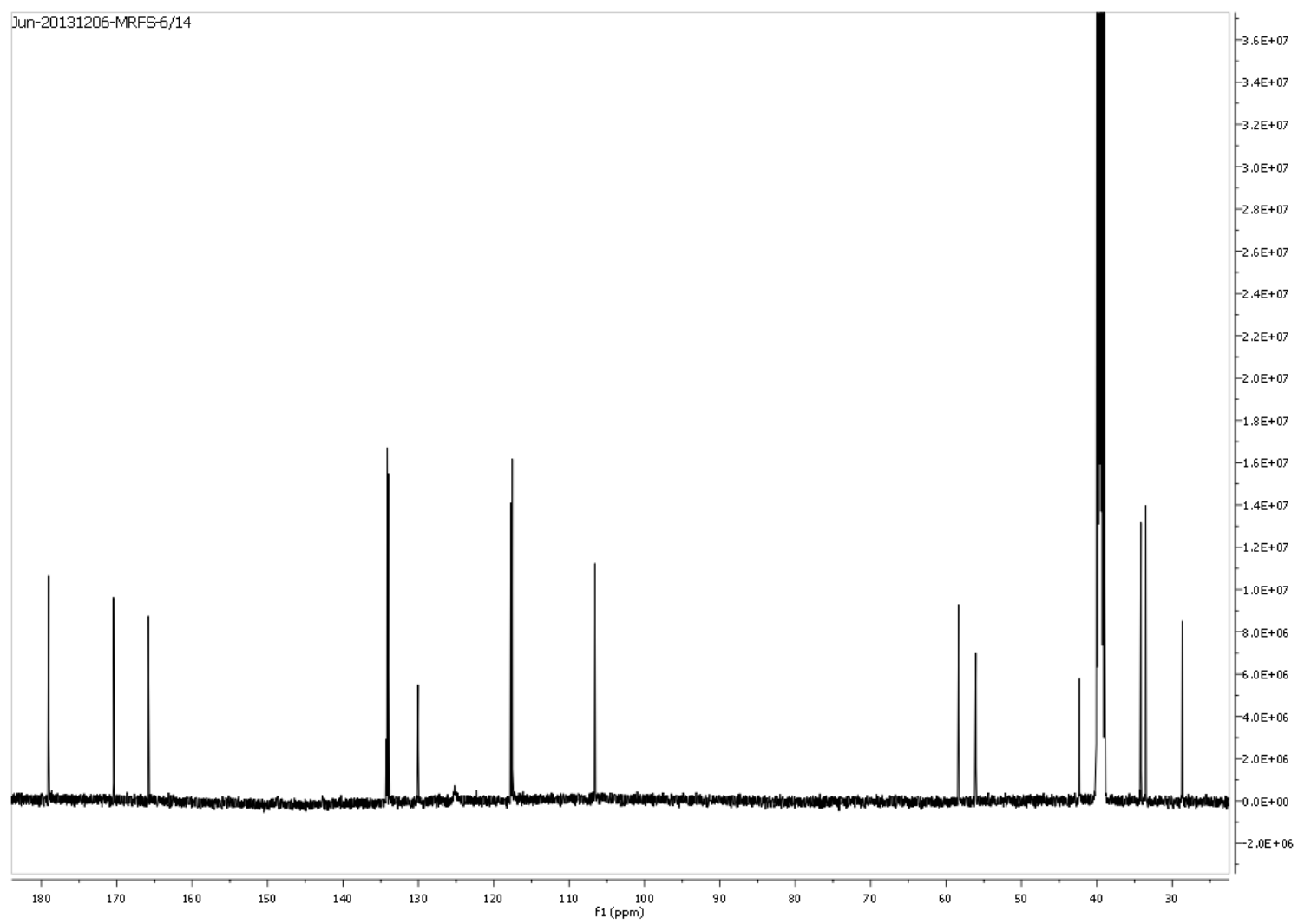

Figure S61. ${ }^{13} \mathrm{C}$ NMR spectrum (DMSO- $d_{6}, 125 \mathrm{MHz}$ ) 


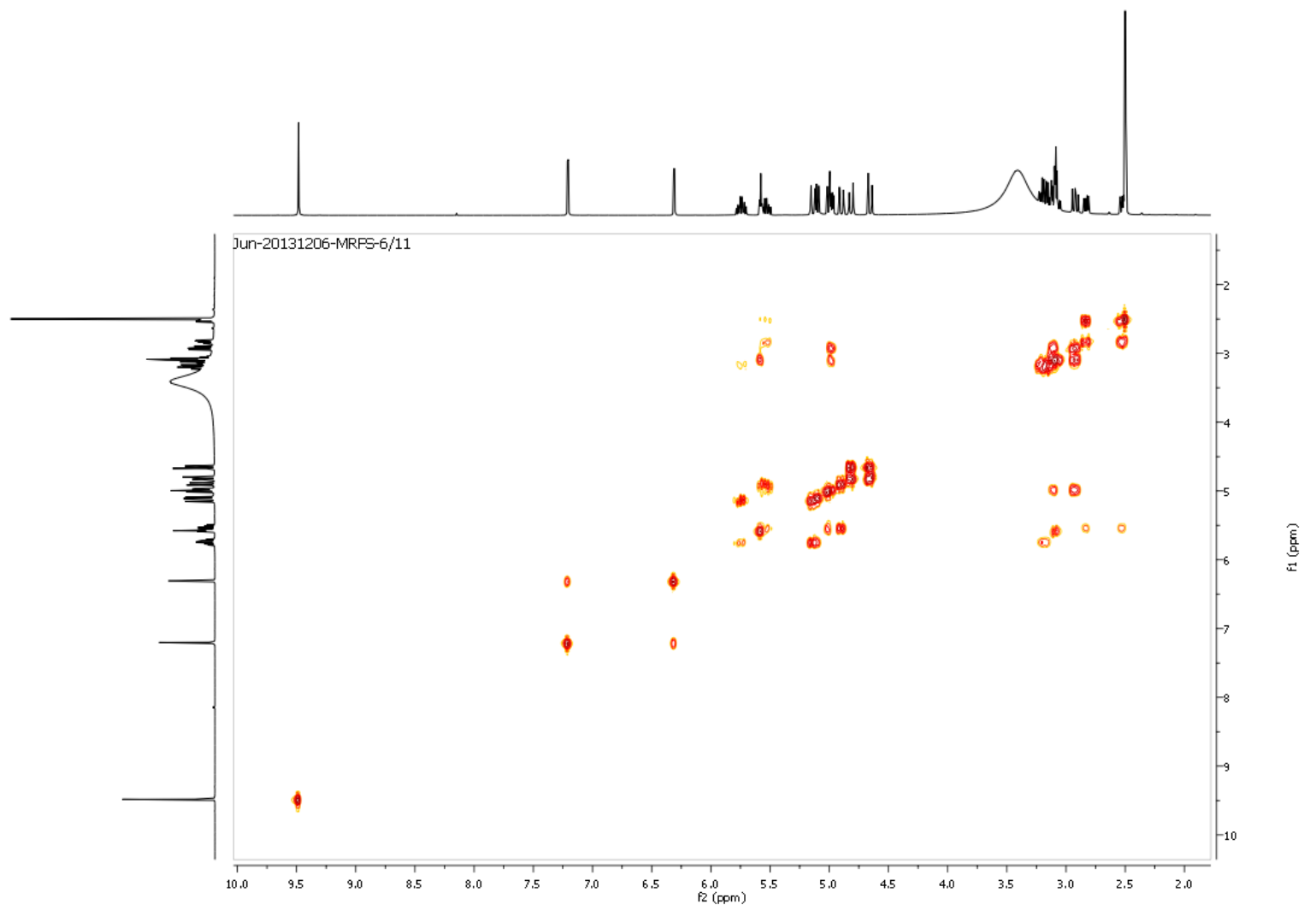

Figure S62. COSY correlations

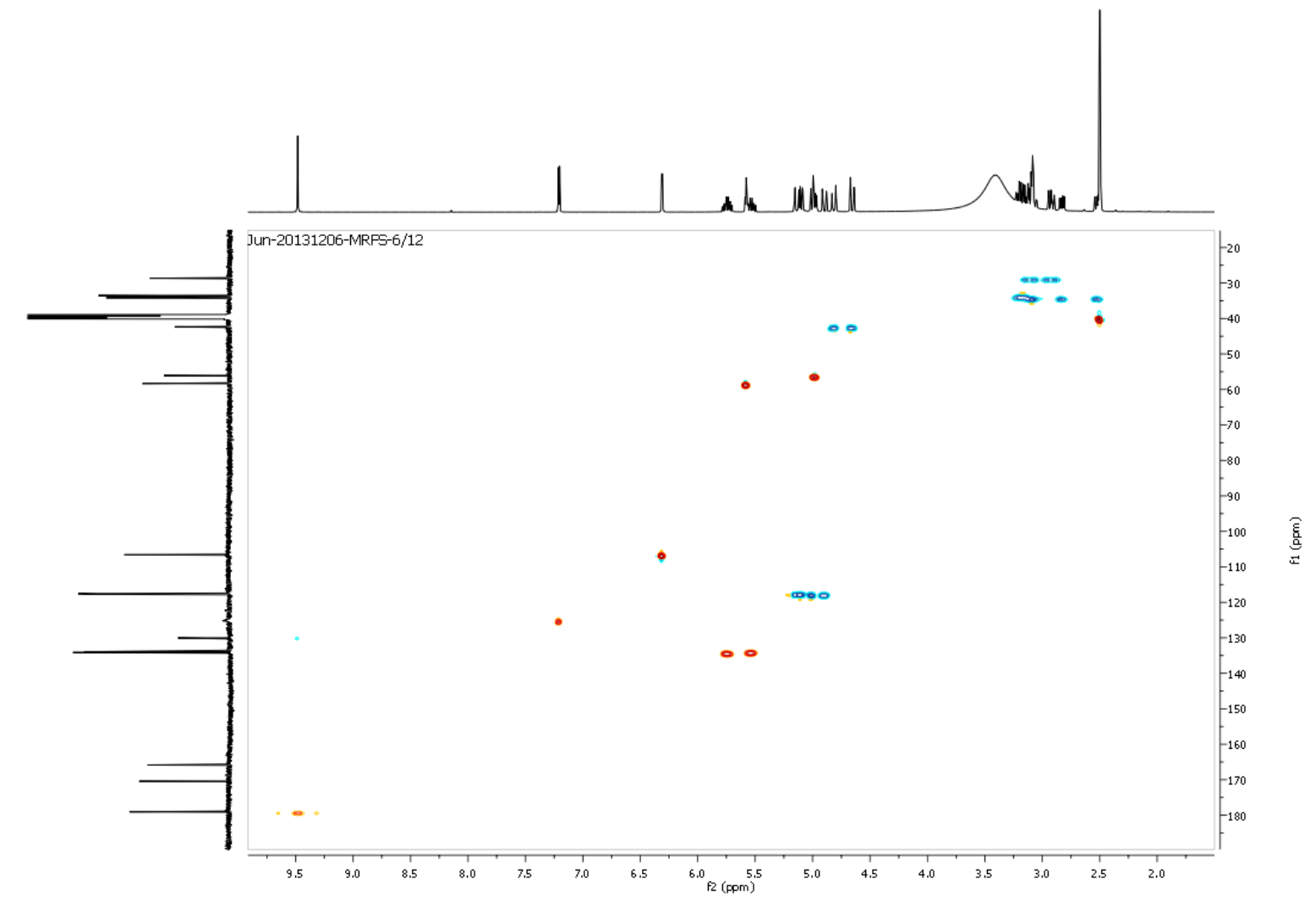

Figure S63. HSQC correlations 


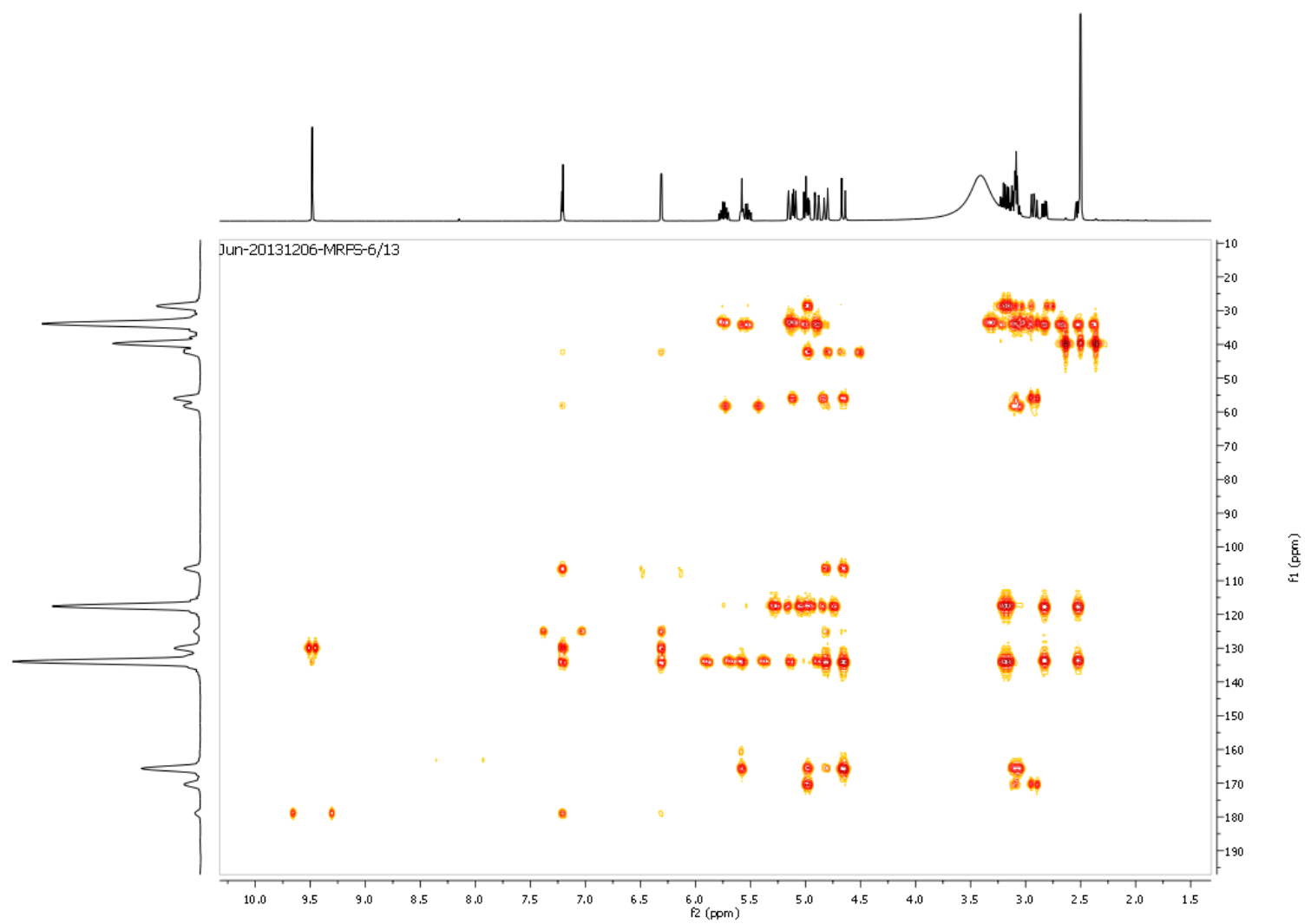

Figure S64. HMBC correlations

2-(allylthioethyl)-4-(allylthiomethyl)-3-oxo-3,4-dihydropyrrolo[1,2a]pyrazin -2(1H)-6-carbaldehyde (racemate of compound 10 and 11)

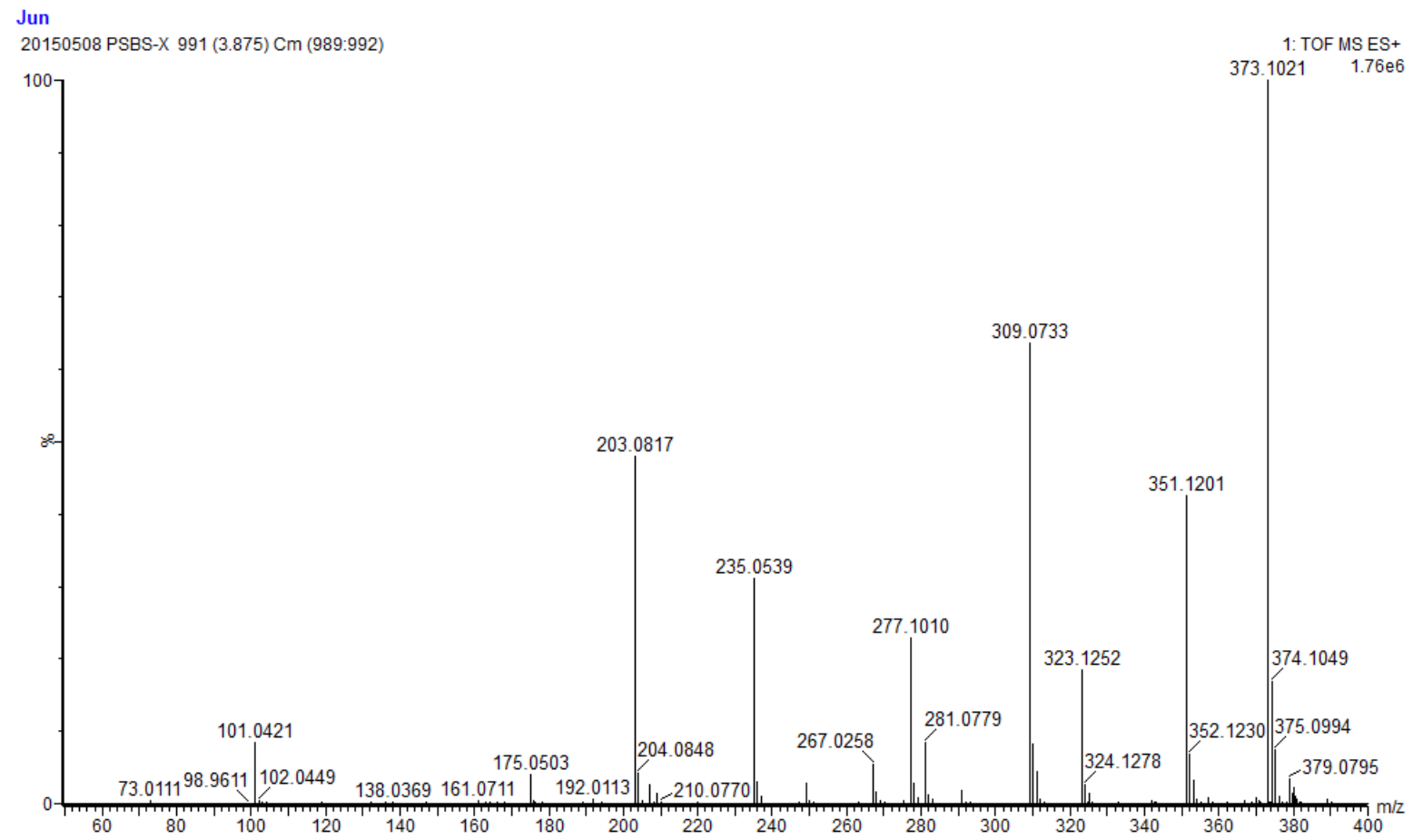

Figure S64. HRESIMS (negative) spectrum 


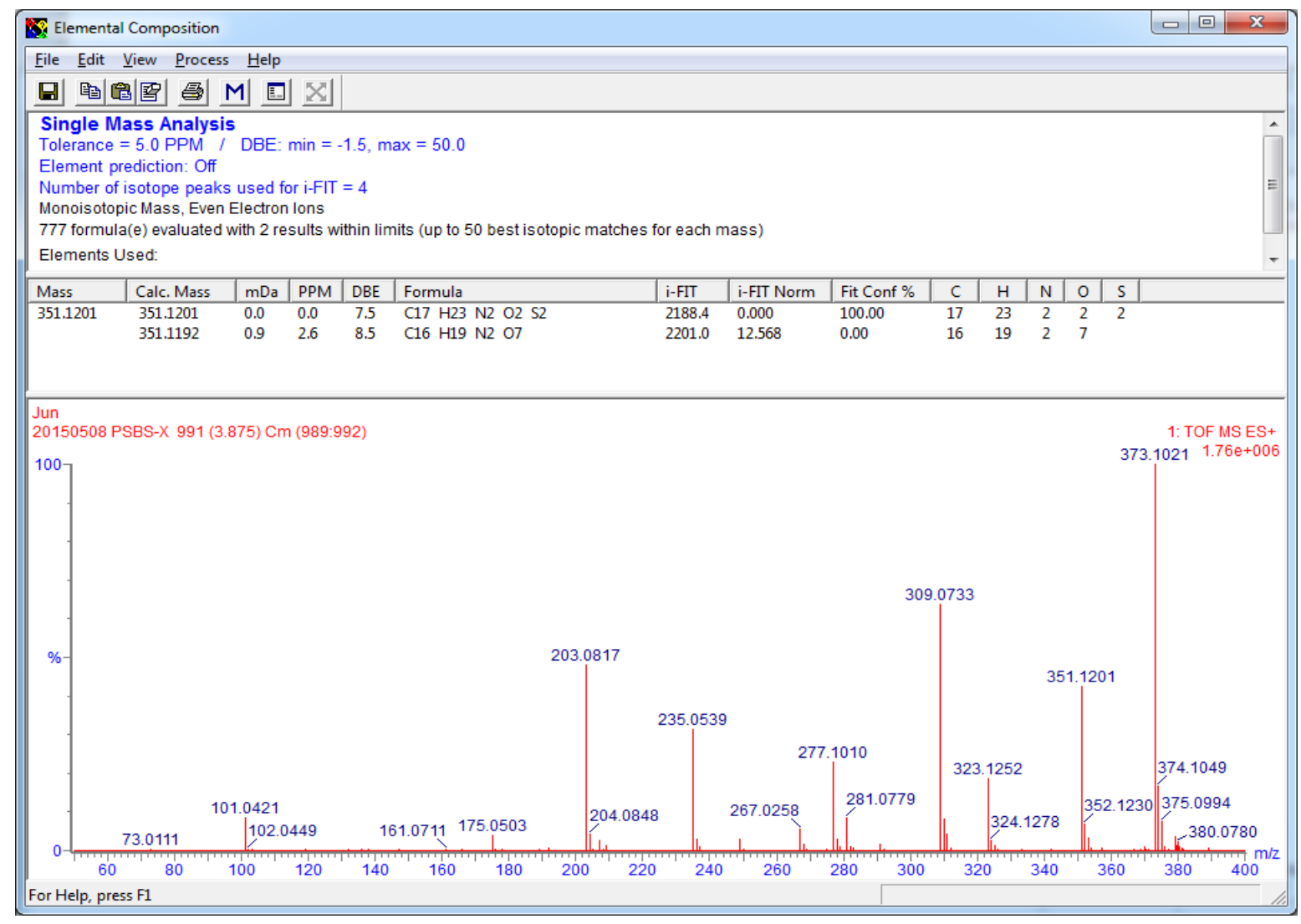

Figure S65. Elemental composition report of the precursor ion 


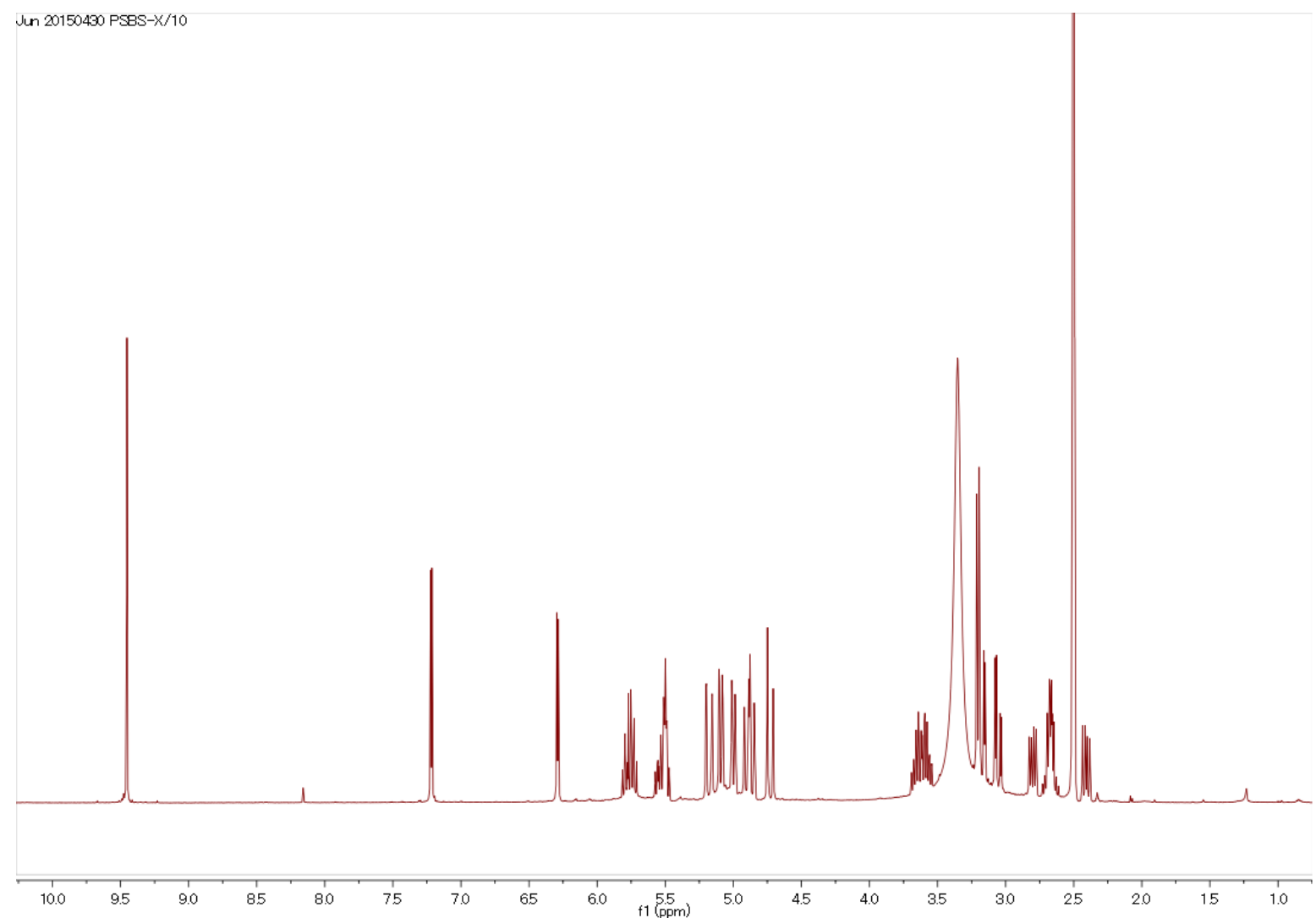

Figure S66. ${ }^{1} \mathrm{H}$ NMR spectrum (DMSO- $d_{6}, 400 \mathrm{MHz}$ )

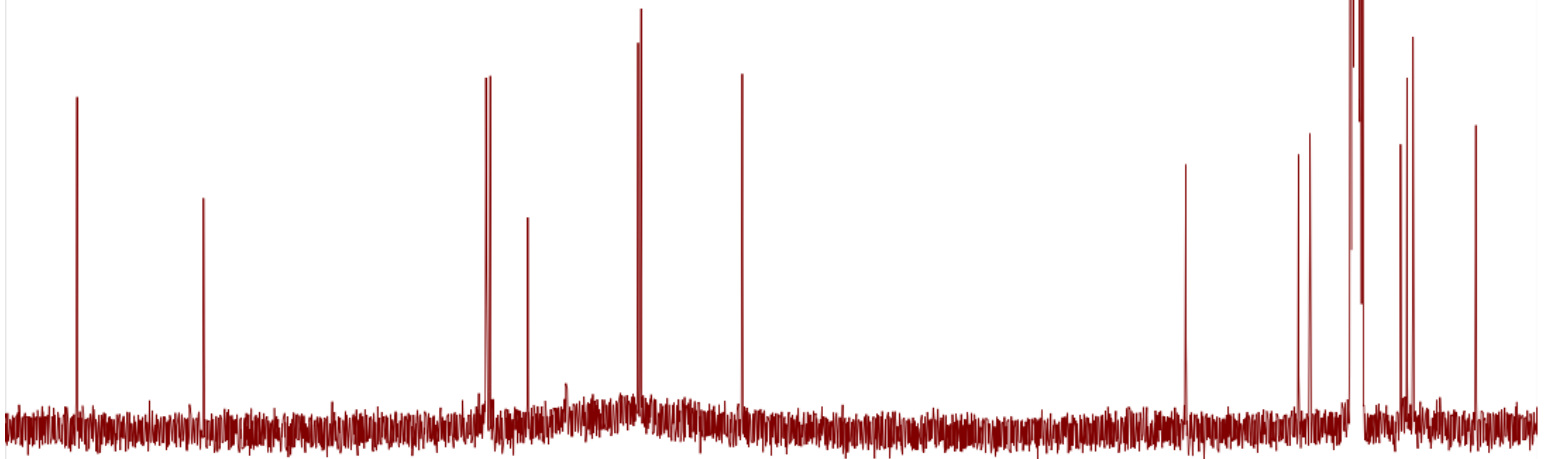

Figure $567 .{ }^{13} \mathrm{C}$ NMR spectrum (DMSO- $d_{6}, 100 \mathrm{MHz}$ ) 


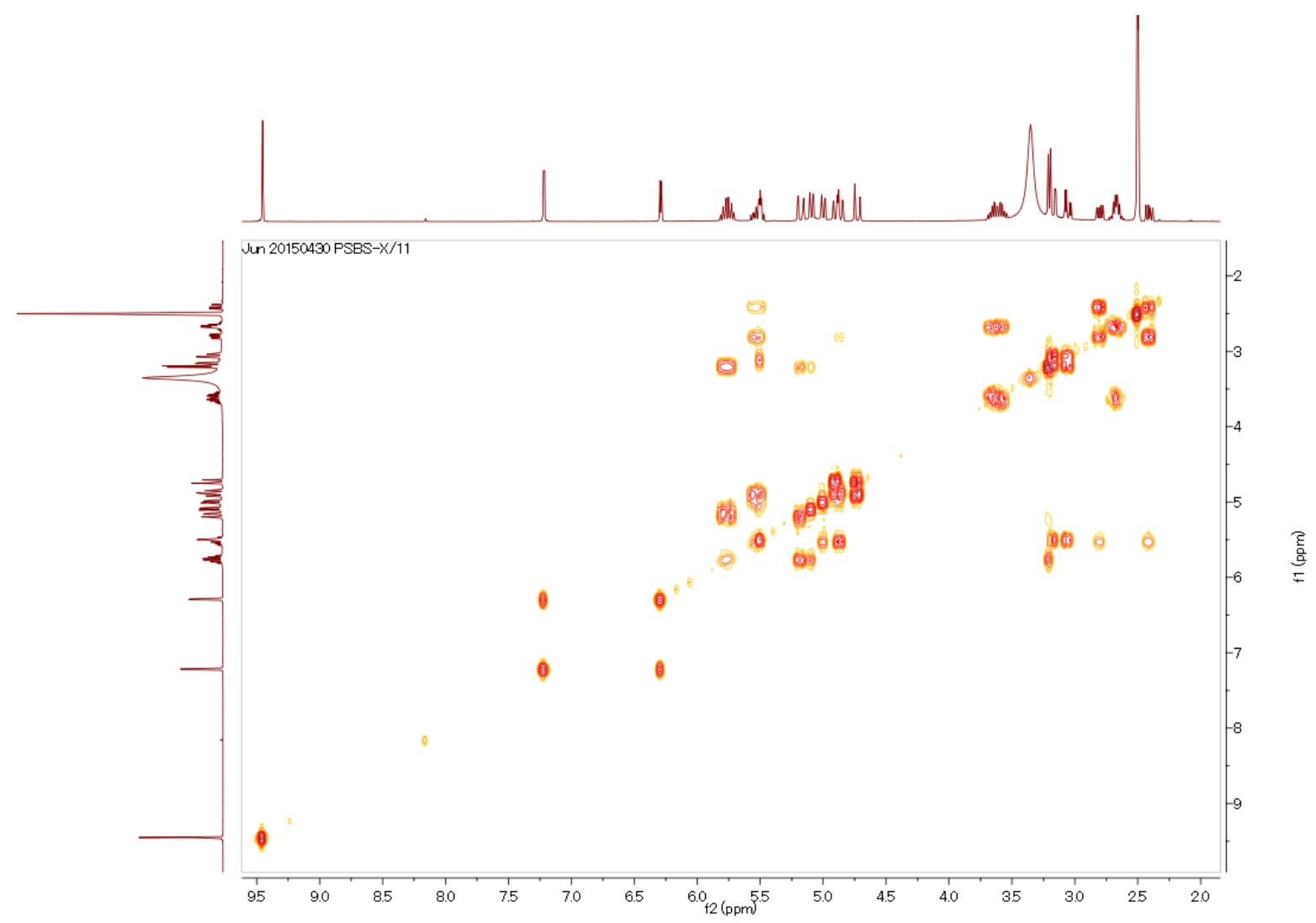

Figure S68. COSY correlations

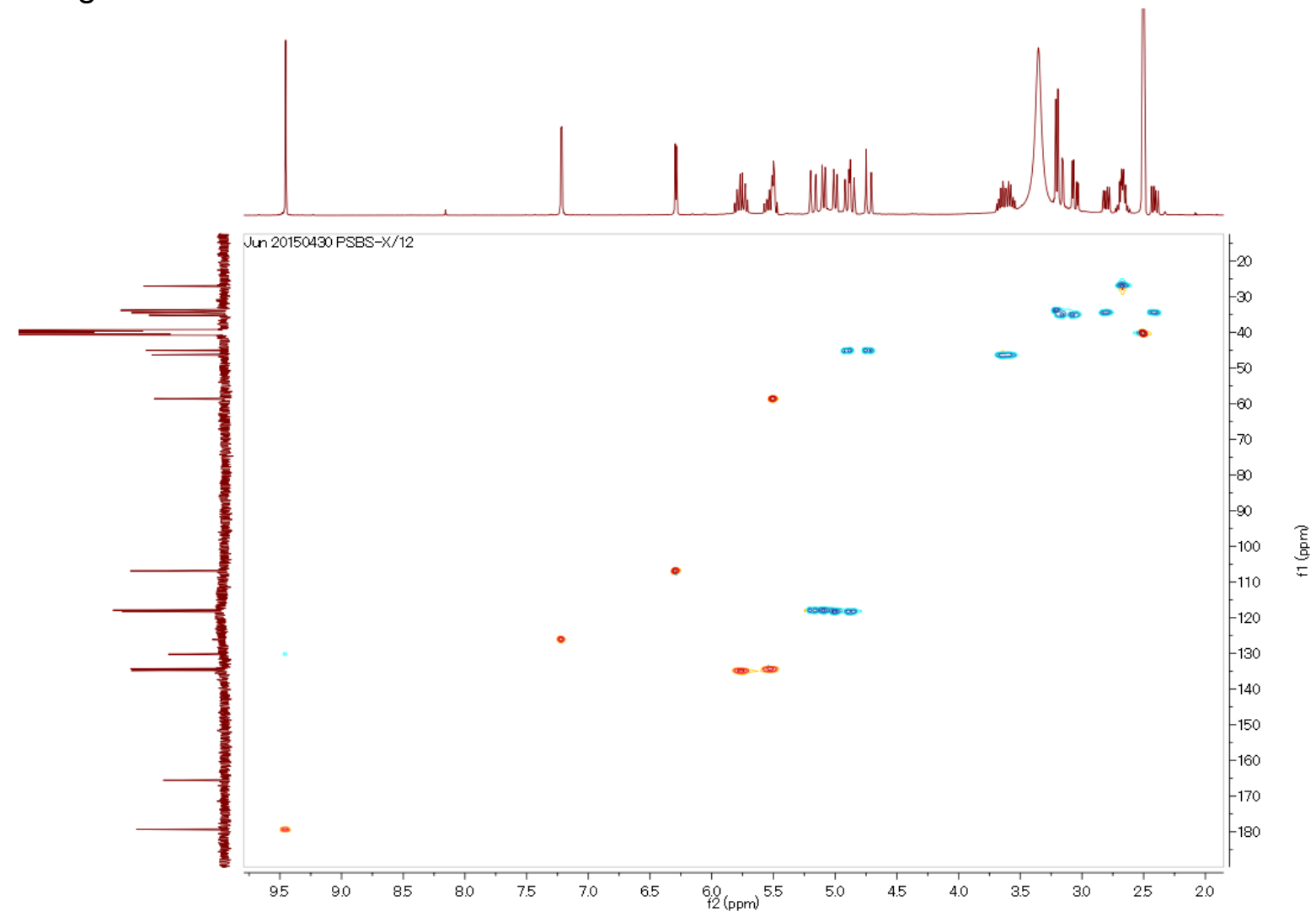

Figure S69. HSQC correlations 


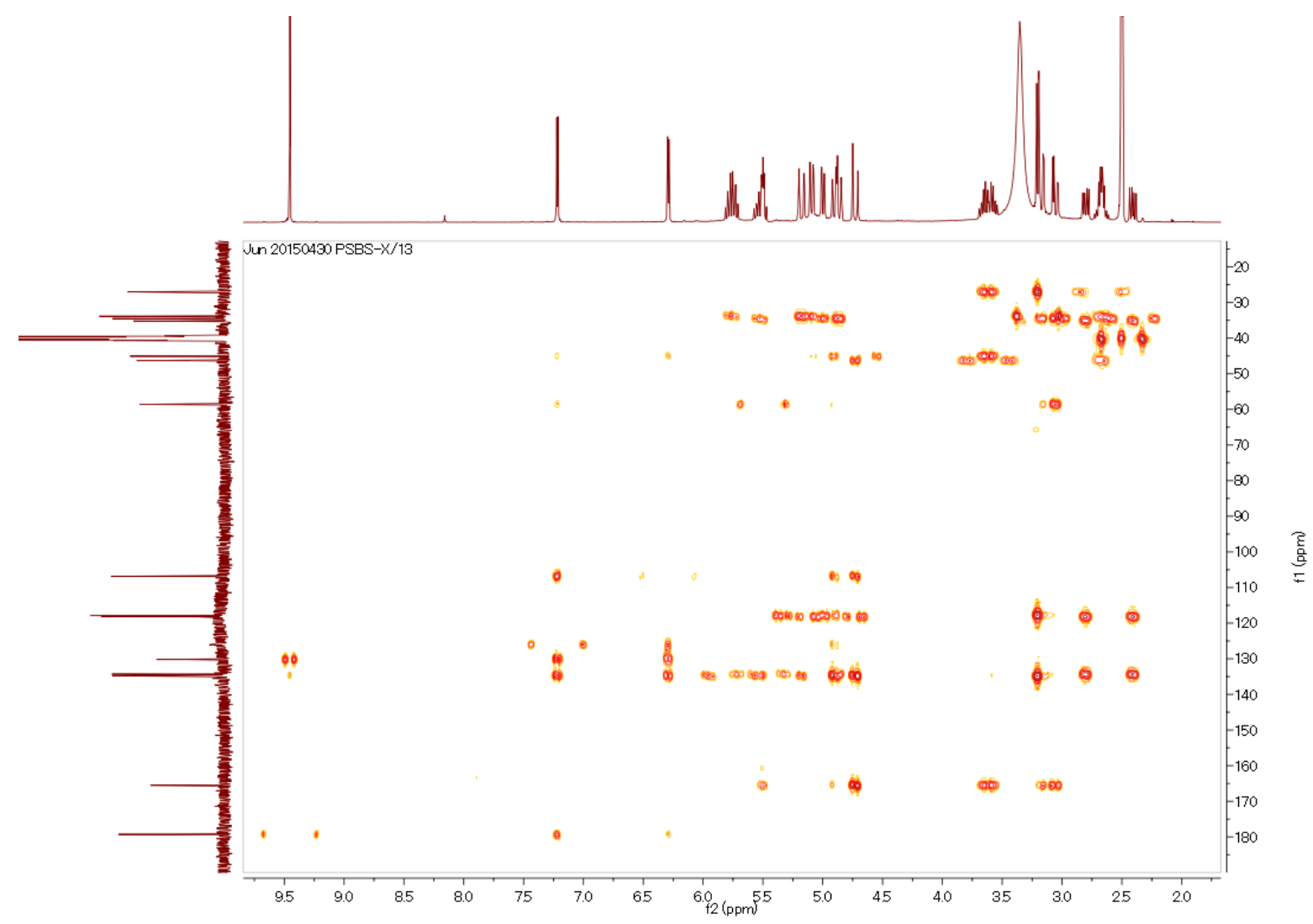

Figure S70. HMBC correlations

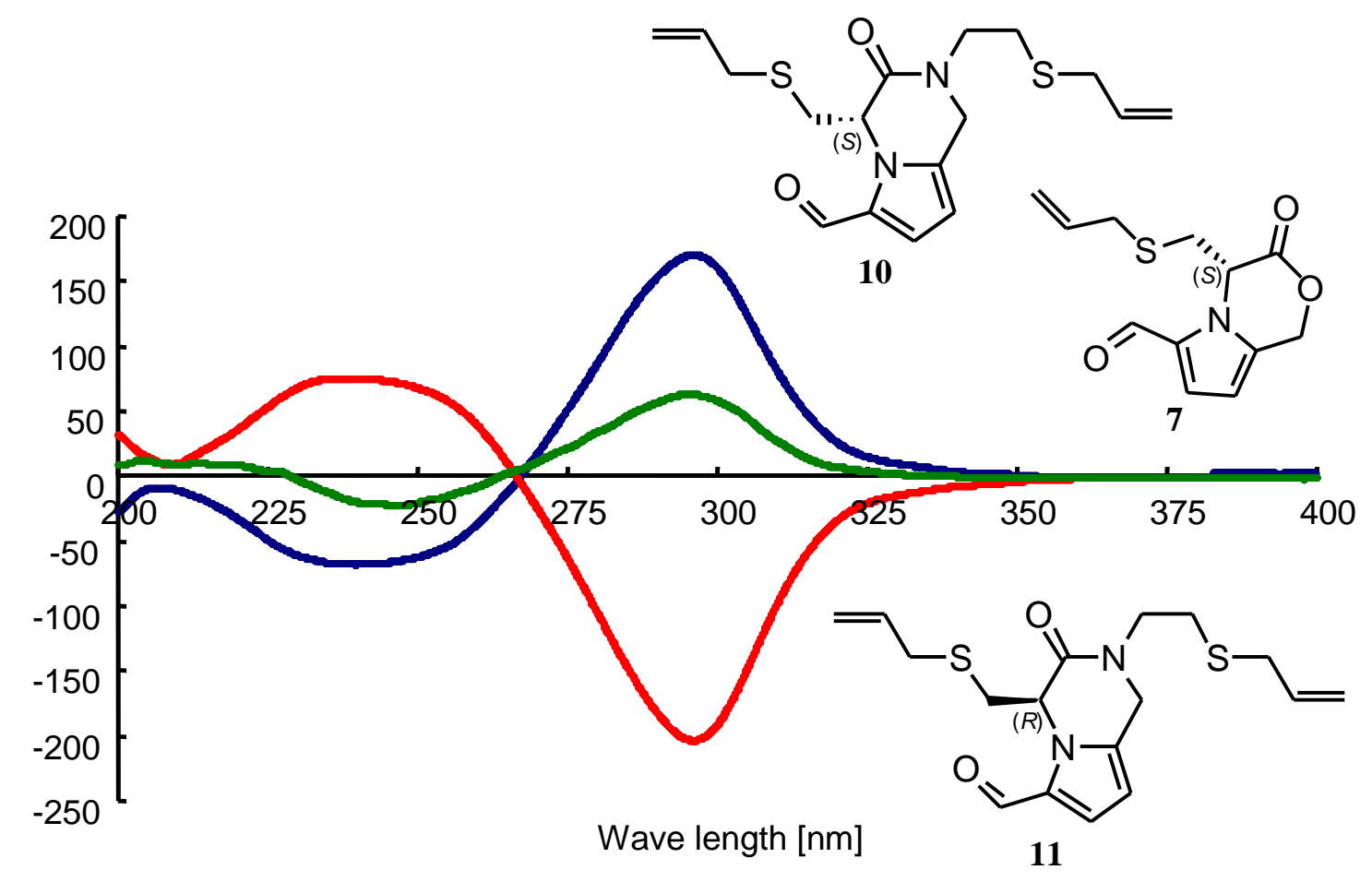

Figure S71. CD spectra of Maillard reaction product 7 (green) and synthesized references 10 (blue) and 11 (red) 

3. Analytical conditions for quantitative analysis on identified compounds in garlic preparations

\begin{tabular}{|c|c|c|c|c|c|c|}
\hline Compounds & MW & Gradient conditions & $\begin{array}{c}\text { Mass transition } \\
(\mathrm{m} / \mathrm{z}) \\
\end{array}$ & $\begin{array}{l}\text { Retention time } \\
\text { (min) }\end{array}$ & $\begin{array}{c}\text { Cone voltage } \\
\text { (V) }\end{array}$ & $\begin{array}{c}\text { Collision energy } \\
(\mathrm{eV}) \\
\end{array}$ \\
\hline 1 & 253 & & $254.2 \rightarrow 206.1$ & 4.47 & 4.0 & 10.0 \\
\hline 2 & 253 & $10 \% \mathrm{~B} \rightarrow 20 \% \mathrm{~B}$ & $254.2 \rightarrow 206.1$ & 4.92 & 4.0 & 10.0 \\
\hline 3 & 253 & & $254.2 \rightarrow 206.1$ & 5.22 & 4.0 & 10.0 \\
\hline 4 & 253 & & $254.2 \rightarrow 206.1$ & 5.71 & 4.0 & 10.0 \\
\hline 5 & 235 & & $258.3 \rightarrow 200.1$ & 5.99 & 35.0 & 11.0 \\
\hline 6 & 269 & $30 \% \mathrm{~B} \rightarrow 60 \% \mathrm{~B} \rightarrow 99 \% \mathrm{~B}$ & $270.2 \rightarrow 228.1$ & 2.00 & 26.0 & 8.0 \\
\hline 7 & 251 & $4.2 \mathrm{~min}$ & $252.2 \rightarrow 178.0$ & 3.24 & 22.0 & 16.0 \\
\hline 8 & 394 & & $395.2 \rightarrow 99.1$ & 3.79 & 30.0 & 20.0 \\
\hline 9 & 394 & & $395.2 \rightarrow 99.1$ & 3.91 & 30.0 & 20.0 \\
\hline
\end{tabular}

Table S2. UPLC-MS/MS (ESI) parameters for quantitative analysis on identified compounds. 